Supporting Information for

\title{
Muscle-Mimetic Synergistic Covalent and Supramolecular Polymers: Phototriggered Formation Leads to Mechanical Performance Boost
}

Zhaoming Zhang, ${ }^{\perp}$ Lin Cheng, ${ }^{\perp}$ Jun Zhao, Hao Zhang, Xinyang Zhao, Yuhang Liu, Ruixue Bai, Hui Pan, Wei Yu, and Xuzhou Yan*

School of Chemistry and Chemical Engineering, Frontiers Science Center for Transformative Molecules, Shanghai Jiao Tong University, Shanghai 200240, P. R. China

${ }^{\perp}$ These authors contributed equally to this work.

*To whom correspondence should be addressed. E-mail: xzyan@sjtu.edu.cn 


\section{Materials and general methods}

All reagents were commercially available and used as supplied without further purification. Deuterated solvents were purchased from Cambridge Isotope Laboratory (Andover, MA). Compounds $\mathbf{4}^{\mathrm{S} 1}, \mathbf{5}^{\mathrm{S} 2}, \mathbf{8}^{\mathrm{S} 3, \mathrm{~S} 4}, \mathbf{1 1}^{\mathrm{S} 5}$ and $\mathbf{1} 7^{\mathrm{S} 6}$ were prepared according to the established methods. NMR spectra were recorded with a Bruker Avance DMX 400 spectrophotometer with use of the deuterated solvent as the lock and the residual solvent or TMS as the internal reference. Solid-state NMR spectra were recorded at ambient pressure on a Bruker AVANCE NEO 600 spectrometer using a standard Bruker magic angle-spinning (MAS) probe with $3.2 \mathrm{~mm}$ (o.d.) zirconia rotors. Melting point of solid compounds were tested on a WRX-4 micro melting point apparatus (Shanghai Yice instrument Co., LTD, China). High resolution mass spectra were obtained on a Bruker SolariX 7.0T FT-ICR MS spectrometer. Gel permeation chromatograph (GPC) was obtained on a HLC-8320GPC (TOSOH, Japan) instrument using dimethylformamide (DMF) as eluent with polystyrene standards. The thermal stability analysis was conducted using a TA Instruments Q500 thermogravimetric analyzer (TGA) under the nitrogen. Each sample $(\sim 5 \mathrm{mg})$ was heated from 50 to $800{ }^{\circ} \mathrm{C}$ with a rate of $20{ }^{\circ} \mathrm{C} / \mathrm{min}$. Transition temperatures of materials determined on a TA Instruments Q2000 differential scanning calorimetry (DSC) under the nitrogen. Dynamic thermomechanical analysis (DMA) was carried out with a dynamic mechanical thermal analyzer (Discovery DMA 850, TA, USA) in the tensile mode at a frequency of $1.0 \mathrm{~Hz}$. Rheological experiments were carried out using a TA Instruments ARES G2 stress-controlled rheometer with an $8 \mathrm{~mm}$ parallel plate attachment. Atomic force microscope (AFM) measurements were performed in a PeakForce QNM (Quantitative Nano-Mechanics) mode on a Bruker 9Bio-FastScan AFM at ambient conditions. Deprotection of UPy moiety was carried out by irradiating UV light $\left(365 \mathrm{~nm}, 70 \mathrm{~mW} / \mathrm{cm}^{2}\right)$ from a UV-LED device (CSM-C6120200JTDX, IUVOT, China).

Mechanical tests: The mechanical properties of the polymers were measured using an Instron 3343 machine in standard stress/strain experiments. As for the preparations of the specimens used for the tests, the CPs and SPs were mixed and irradiated in solution, then the resultant CSPs were dried and further molded by hot-pressing the samples into Teflon molds at $70{ }^{\circ} \mathrm{C}$. Young's modulus was determined from the initial slope of the stress-strain curves. Toughness 
was obtained by integrating the area under stress strain curve. Energy dissipation was calculated by integrating the area encompassed by the cyclic tensile curves. Damping capacity was defined as the ratio of the dissipated energy (the area encompassed by the loading and unloading curves) to the loading energy (the area encompassed by the loading curve).

\section{Characterization of photo-induced integration of CPS and SPs in the solid state:}

For the photographs, the CPs and SPs were mixed and dried, and then molded by hot-pressing. The photographs of the specimen before and after $2 \mathrm{~h}$ irradiation were taken.

For the AFM measurements, rectangular film of the mixed CPs and SPs was formed by slowly evaporating the solvent of chloroform. Half of the specimen was wrapped by aluminium foil during the irradiation. Then the two regions of the specimen, namely the regions with and without irradiation, were measured by AFM.

For the in situ rheology characterization, the dried sample was pressed into a thin film with a thickness of $3.2 \mu \mathrm{m}$. The rheology test was carried out using a Kinexus Ultra rotational rheometer (NETZSCH Instruments) equipped with a LED device with the wavelength of 365 $\mathrm{nm}$. The top fixture was made from quartz, and the irradiation from the LED device passes through the quartz window onto the sample.

For the tensile tests, the dried sample was molded by hot-pressing to prepare two dumbbellshaped specimens. Tensile test of one sample was conducted directly, that is, the measurement of sample before irradiation. And the tensile test of the other specimen was performed after a 2 $\mathrm{h}$ irradiation.

Photo-enhanced self-healing experiments: Two specimens were separately cut into two completely separate pieces, and the cut faces were gently pressed together. One sample was irradiated for $2 \mathrm{~h}$, and the other sample was self-healed without irradiation. 


$$
\begin{aligned}
& \text { 等 }
\end{aligned}
$$

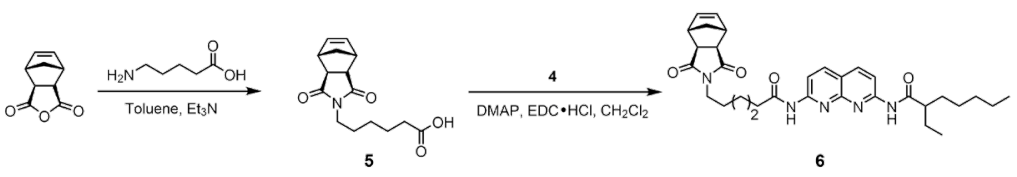

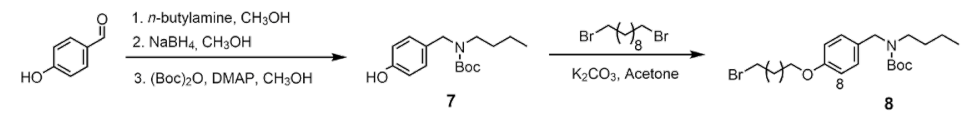

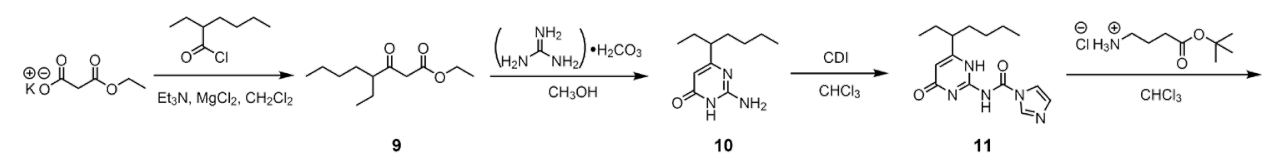
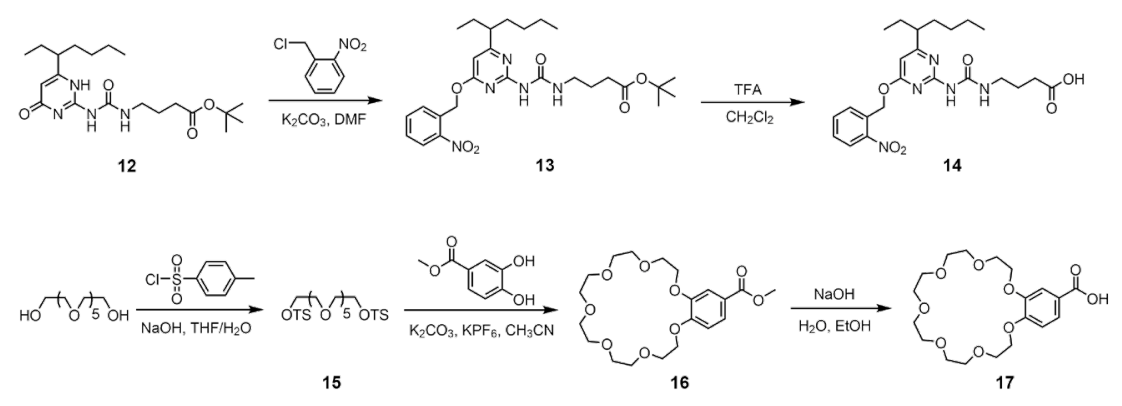

15

$$
\underset{\text { DMAP. EDC } \cdot \mathrm{HCI} \mathrm{CH}_{2} \mathrm{Cl}_{2}}{\longrightarrow}
$$

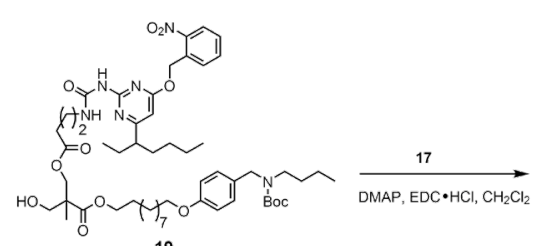

18

19

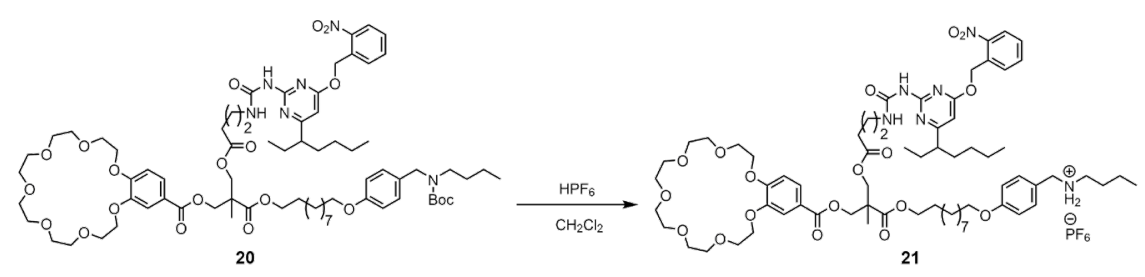

Scheme S1. Synthetic route to the monomers for CPs and SP. 

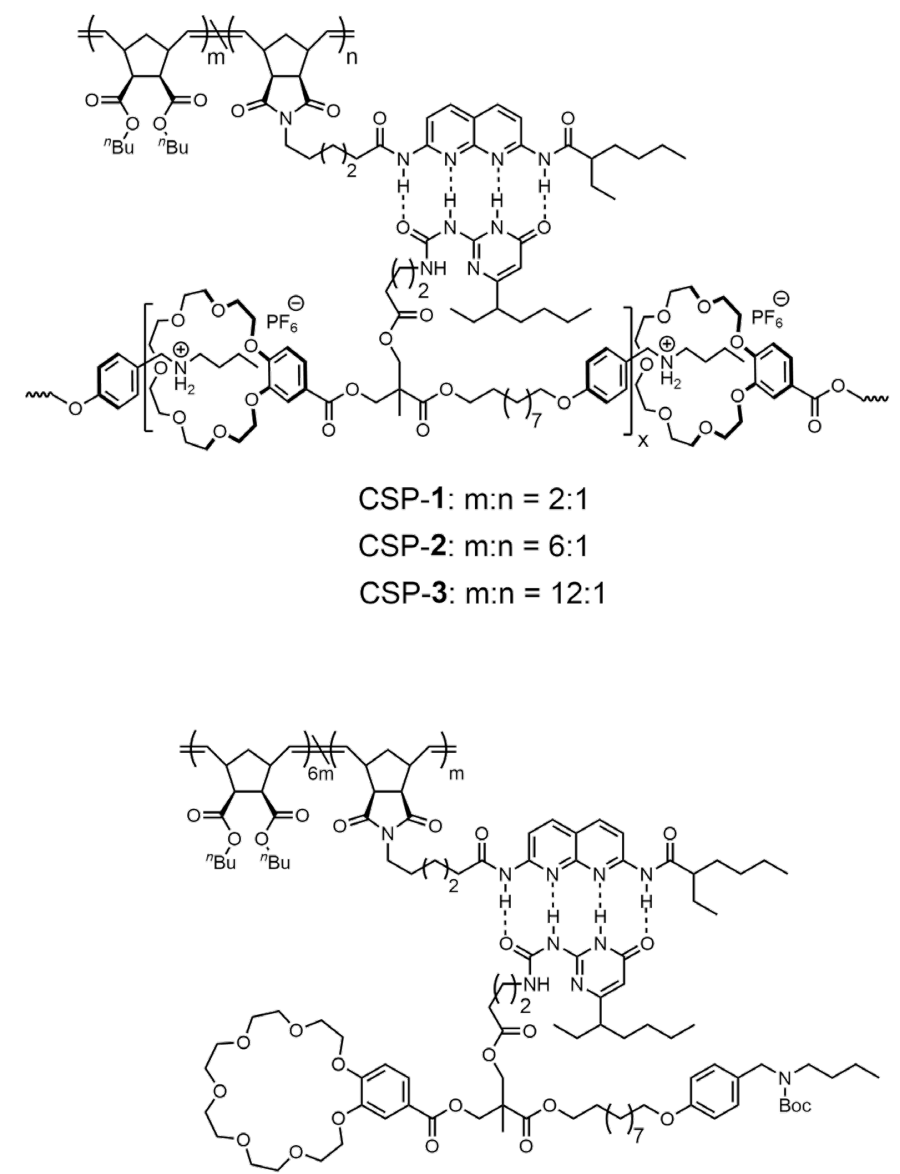

Control-1
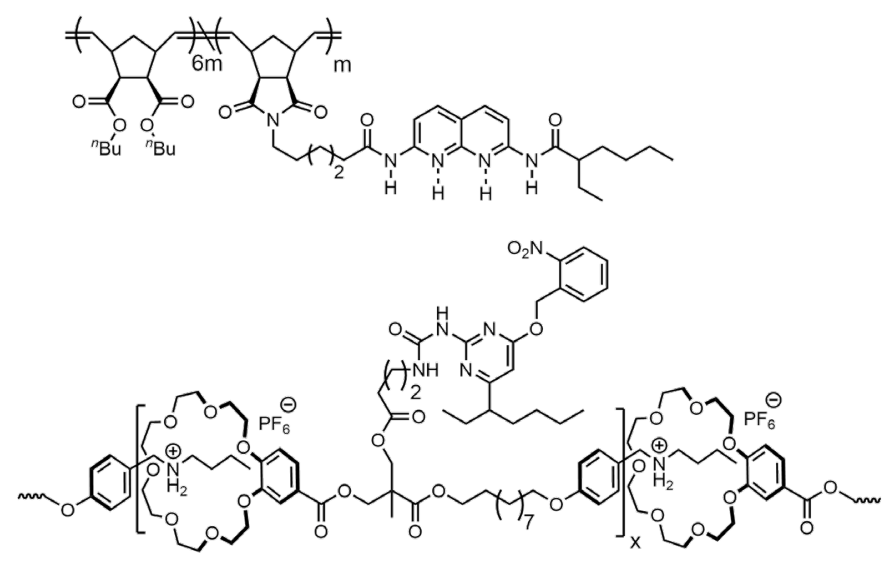

Control-2

Scheme S2. Chemical structures of CSPs-1-3 and controls-1 and 2. 


\section{Synthesis of compound 6}

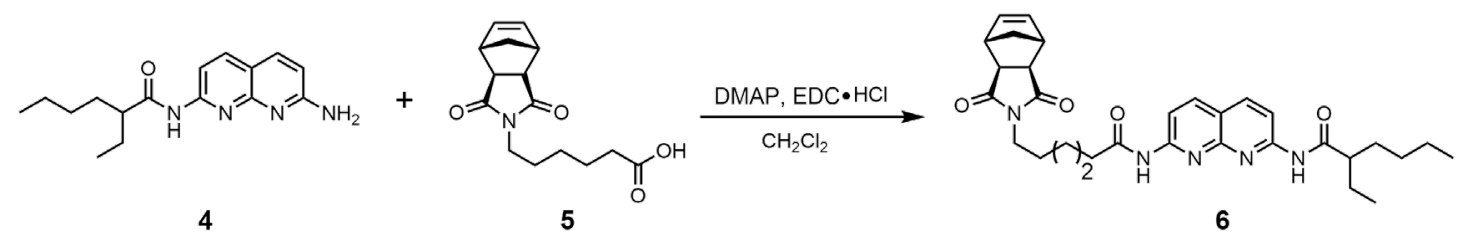

A stirred solution of compound 4 (3.52 g, $12.6 \mathrm{mmol})$, DMAP (230 mg, $1.89 \mathrm{mmol})$, compound 5 (3.52 g, $12.6 \mathrm{mmol})$, in $\mathrm{CH}_{2} \mathrm{Cl}_{2}(60 \mathrm{~mL})$, in a round-bottomed flask with magnetic stir-bar was cooled to $0{ }^{\circ} \mathrm{C}$ via an ice-water bath. $\mathrm{EDC} \cdot \mathrm{HCl}(2.40 \mathrm{~g}, 12.5 \mathrm{mmol})$ was added and the reaction was allowed to warm to ambient temperature over several hours and was kept for 14 h. The solution was diluted with $\mathrm{CH}_{2} \mathrm{Cl}_{2}$ and washed with water, saturated aqueous sodium bicarbonate, and finally with brine. The organic phase was dried using $\mathrm{Na}_{2} \mathrm{SO}_{4}$ and the solvent was removed under vacuum. The crude was further purified by gel chromatography to afford compound 6 as a white powder $\left(5.22\right.$ g, 76\%). M.p. $=82{ }^{\circ} \mathrm{C}$. The ${ }^{1} \mathrm{H}$ NMR spectrum of compound 6 is shown in Figure $\mathrm{S} 1 .{ }^{1} \mathrm{H}$ NMR $\left(\mathrm{CDCl}_{3}\right.$, room temperature, $\left.400 \mathrm{MHz}\right) \delta(\mathrm{ppm})$ : $8.47(\mathrm{~d}, J=8.8 \mathrm{~Hz}, 1 \mathrm{H}), 8.41(\mathrm{~d}, J=8.8 \mathrm{~Hz}, 1 \mathrm{H}), 8.28(\mathrm{~d}, J=7.2 \mathrm{~Hz}, 2 \mathrm{H}), 8.13(\mathrm{dd}, J=8.8$, $1.6 \mathrm{~Hz}, 2 \mathrm{H}), 6.27(\mathrm{t}, J=2.0 \mathrm{~Hz}, 2 \mathrm{H}), 3.53-3.42(\mathrm{~m}, 2 \mathrm{H}), 3.27-3.24(\mathrm{~m}, 2 \mathrm{H}), 2.67(\mathrm{~d}, J=1.2$ $\mathrm{Hz}, 2 \mathrm{H}), 2.45(\mathrm{t}, J=7.6 \mathrm{~Hz}, 2 \mathrm{H}), 2.22(\mathrm{~m}, 1 \mathrm{H}), 1.81-1.68(\mathrm{~m}, 4 \mathrm{H}), 1.68-1.48(\mathrm{~m}, 4 \mathrm{H})$, $1.44-1.11(\mathrm{~m}, 8 \mathrm{H}), 0.97(\mathrm{t}, J=7.2 \mathrm{~Hz}, 3 \mathrm{H}), 0.92-0.84(\mathrm{~m}, 3 \mathrm{H})$. The ${ }^{13} \mathrm{C}$ NMR spectrum of compound 6 is shown in Figure S2. ${ }^{13} \mathrm{C}$ NMR $\left(\mathrm{CDCl}_{3}\right.$, room temperature, $\left.100 \mathrm{MHz}\right) \delta(\mathrm{ppm})$ : $178.15,175.58,172.02,153.95,153.61,139.06,139.03,137.80,118.34,113.60,113.46,50.80$, $47.83,45.14,42.75,38.38,37.46,32.35,29.73,27.34,26.36,25.98,24.47,22.72,13.92,11.99$. HRESIMS is shown in Figure S3: $m / z$ calcd for $\mathrm{C}_{31} \mathrm{H}_{39} \mathrm{~N}_{5} \mathrm{O}_{4}, 546.30748[\mathrm{M}+\mathrm{H}]^{+}$; found $546.30493[\mathrm{M}+\mathrm{H}]^{+}$. 


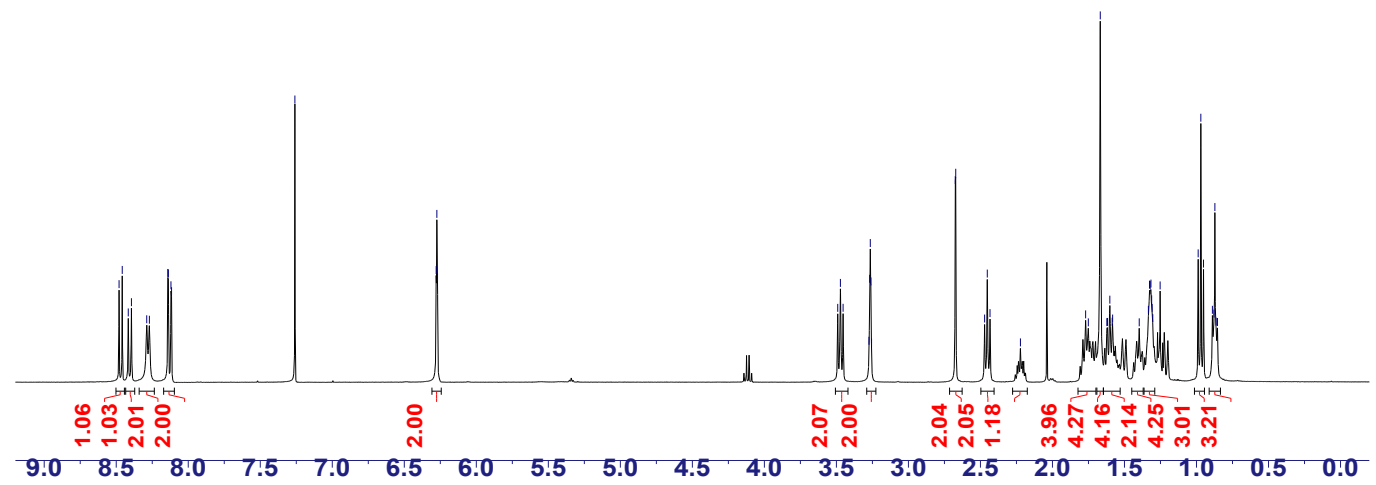

Figure S1. ${ }^{1} \mathrm{H}$ NMR spectrum $\left(\mathrm{CDCl}_{3}\right.$, room temperature, $\left.400 \mathrm{MHz}\right)$ of compound 6 .

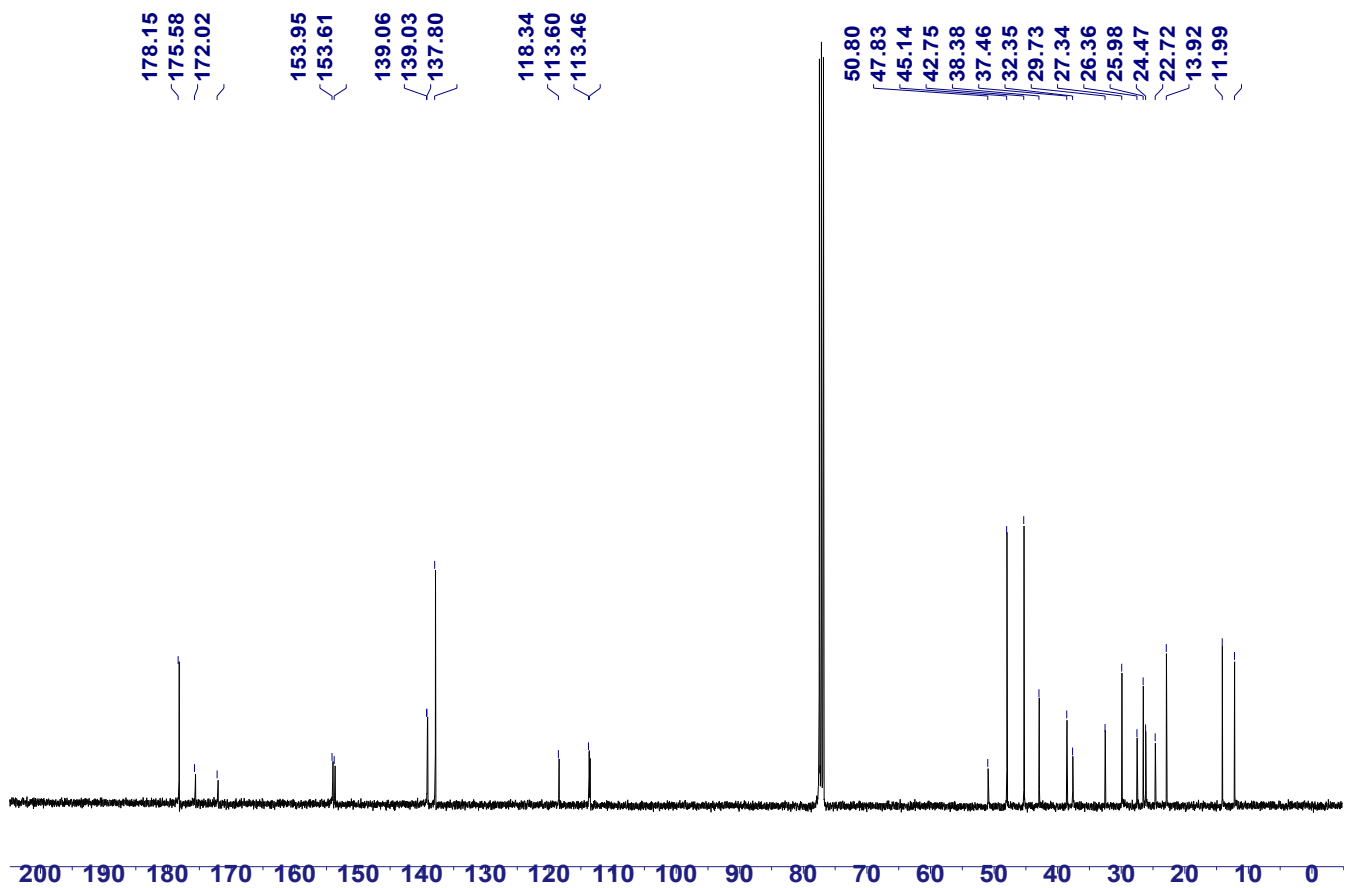

Figure S2. ${ }^{13} \mathrm{C}$ NMR spectrum $\left(\mathrm{CDCl}_{3}\right.$, room temperature, $\left.100 \mathrm{MHz}\right)$ of compound $\mathbf{6}$. 


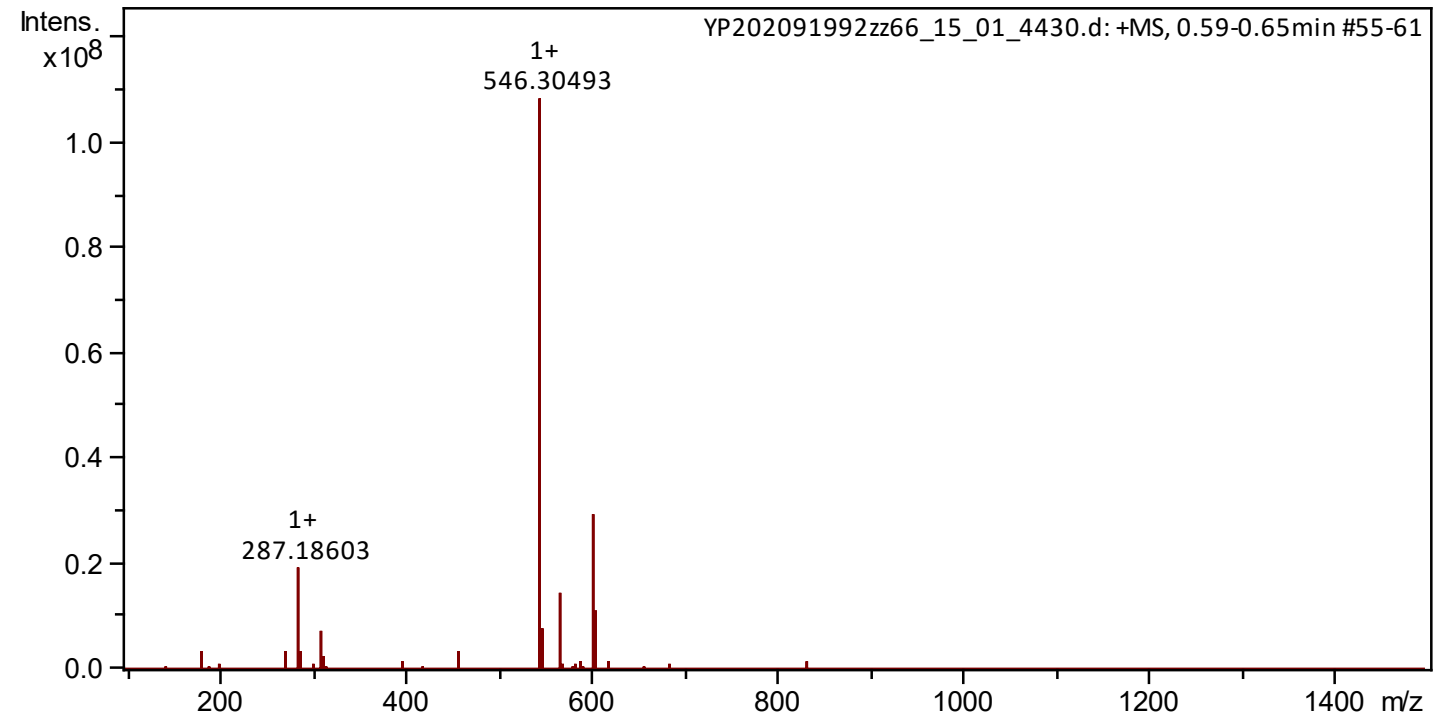

Figure S3. Electrospray ionization mass spectrum of compound 6. 
Synthesis of compound 12

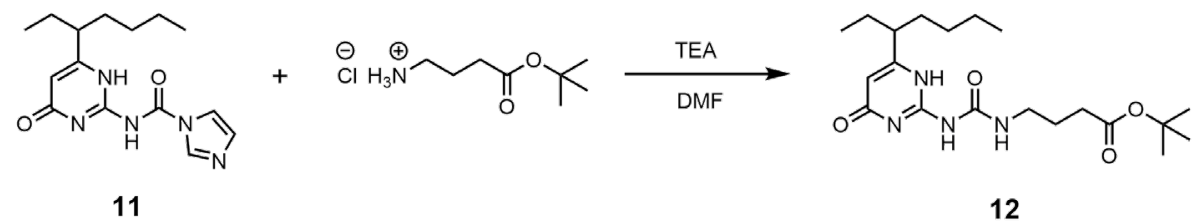

Compound 11 (5.01 g, $16.5 \mathrm{mmol})$ was dissolved in DMF (100 mL), trimethylamine (2.10 mL) and N-(6-methyl-4-oxo-1,4-dihydropyrimidin-2-yl)-1H-imidazole-1-carboxamide (2.48 g, $12.7 \mathrm{mmol}$ ) were added and the mixture was stirred overnight at room temperature under $\mathrm{N}_{2}$. The solvent was evaporated and the solids suspended in chloroform and filtered. The filtrate was washed with water and the organic phase was dried using $\mathrm{Na}_{2} \mathrm{SO}_{4}$, and then purified via gel chromatography to afford compound $\mathbf{1 2}$ as a light yellow oil (4.78 g, 95\%). The ${ }^{1} \mathrm{H}$ NMR spectrum of compound $\mathbf{1 2}$ is shown in Figure S4. ${ }^{1} \mathrm{H}$ NMR $\left(\mathrm{CDCl}_{3}\right.$, room temperature, 400 MHz) $\delta(\mathrm{ppm}): 13.20(\mathrm{~s}, 1 \mathrm{H}), 11.93(\mathrm{~s}, 1 \mathrm{H}), 10.28(\mathrm{~s}, 1 \mathrm{H}), 5.80(\mathrm{~d}, J=1.6 \mathrm{~Hz}, 1 \mathrm{H}), 3.29(\mathrm{~m}$, 2H), $2.30(\mathrm{dd}, J=9.2,6.0 \mathrm{~Hz}, 2 \mathrm{H}), 1.96-1.84(\mathrm{~m}, 2 \mathrm{H}), 1.75(\mathrm{~s}, 1 \mathrm{H}), 1.71-1.49(\mathrm{~m}, 4 \mathrm{H}), 1.43$ $(\mathrm{s}, 9 \mathrm{H}), 1.36-1.16(\mathrm{~m}, 4 \mathrm{H}), 0.92-0.80(\mathrm{~m}, 6 \mathrm{H})$. The ${ }^{13} \mathrm{C}$ NMR spectrum of compound 12 is shown in Figure $\mathrm{S} 5 .{ }^{13} \mathrm{C}$ NMR $\left(\mathrm{CDCl}_{3}\right.$, room temperature, $\left.100 \mathrm{MHz}\right) \delta(\mathrm{ppm}): 173.07,172.42$, $156.76,155.40,154.80,106.23,80.10,45.34,39.22,32.92,32.87,29.29,28.07,26.59,24.94$, 22.44, 13.85, 11.67. HRESIMS is shown in Figure $\mathrm{S} 6: \mathrm{m} / z$ calcd for $\mathrm{C}_{20} \mathrm{H}_{34} \mathrm{~N}_{4} \mathrm{O}_{4}, 395.26528$ $[\mathrm{M}+\mathrm{H}]^{+}$; found $395.26424[\mathrm{M}+\mathrm{H}]^{+}$ 


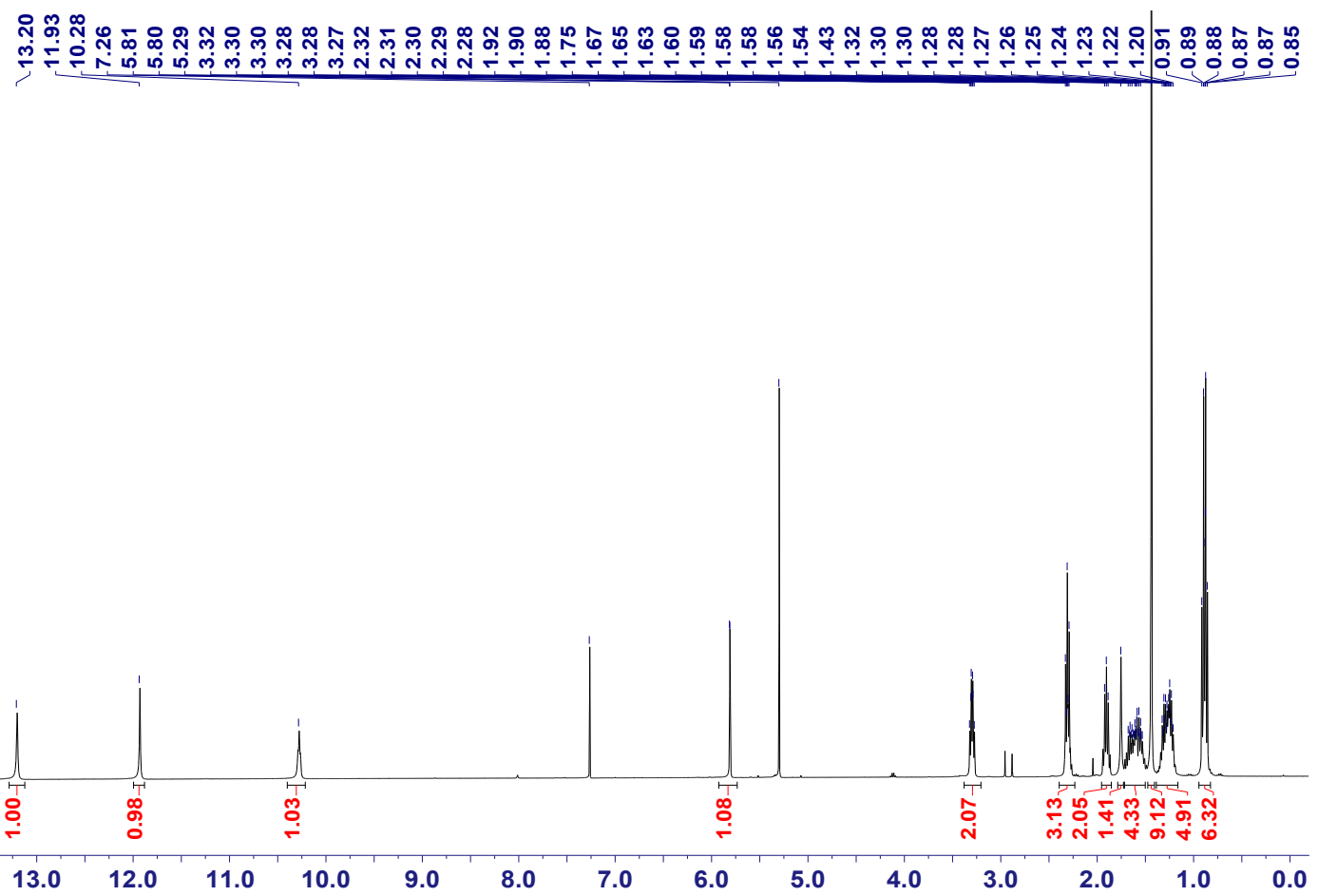

Figure S4. ${ }^{1} \mathrm{H}$ NMR spectrum $\left(\mathrm{CDCl}_{3}\right.$, room temperature, $\left.400 \mathrm{MHz}\right)$ of compound 12.

\begin{tabular}{|c|c|c|c|}
\hline 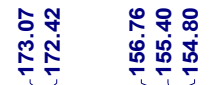 & 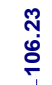 & 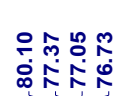 & 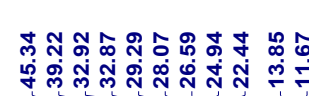 \\
\hline
\end{tabular}

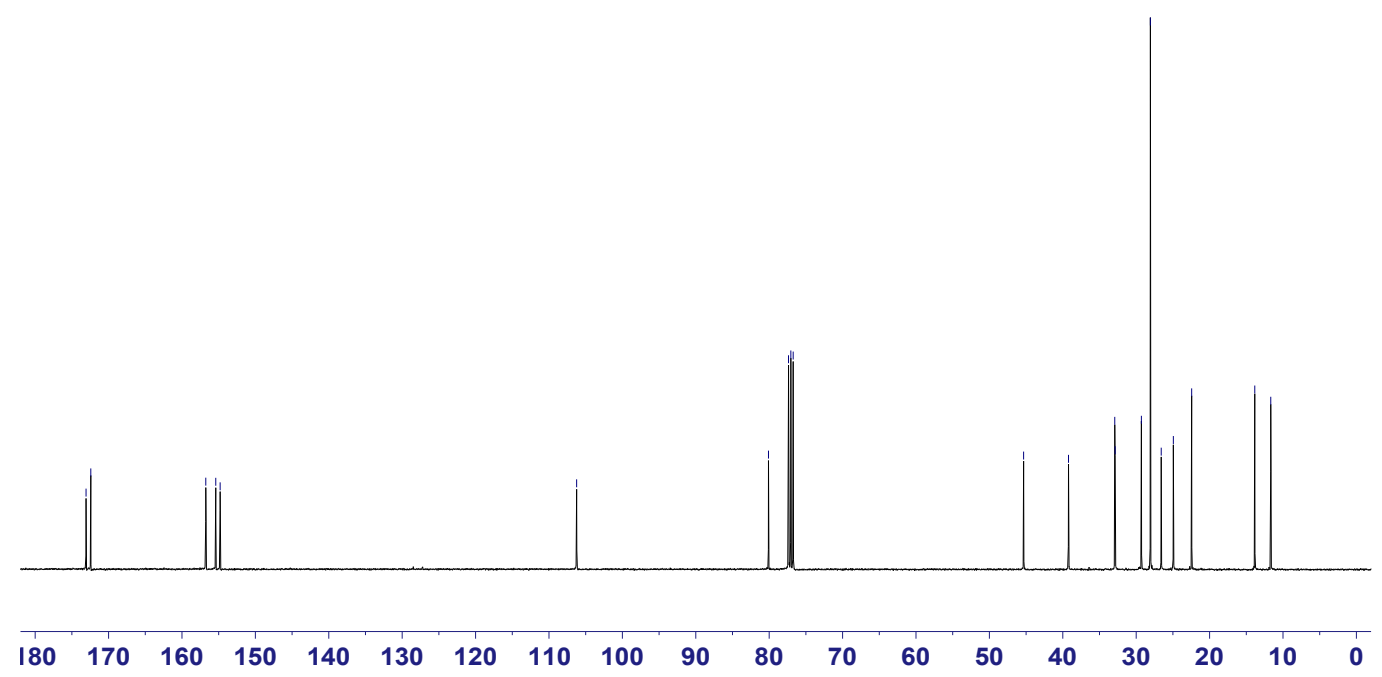

Figure S5. ${ }^{13} \mathrm{C}$ NMR spectrum $\left(\mathrm{CDCl}_{3}\right.$, room temperature, $\left.100 \mathrm{MHz}\right)$ of compound 12. 


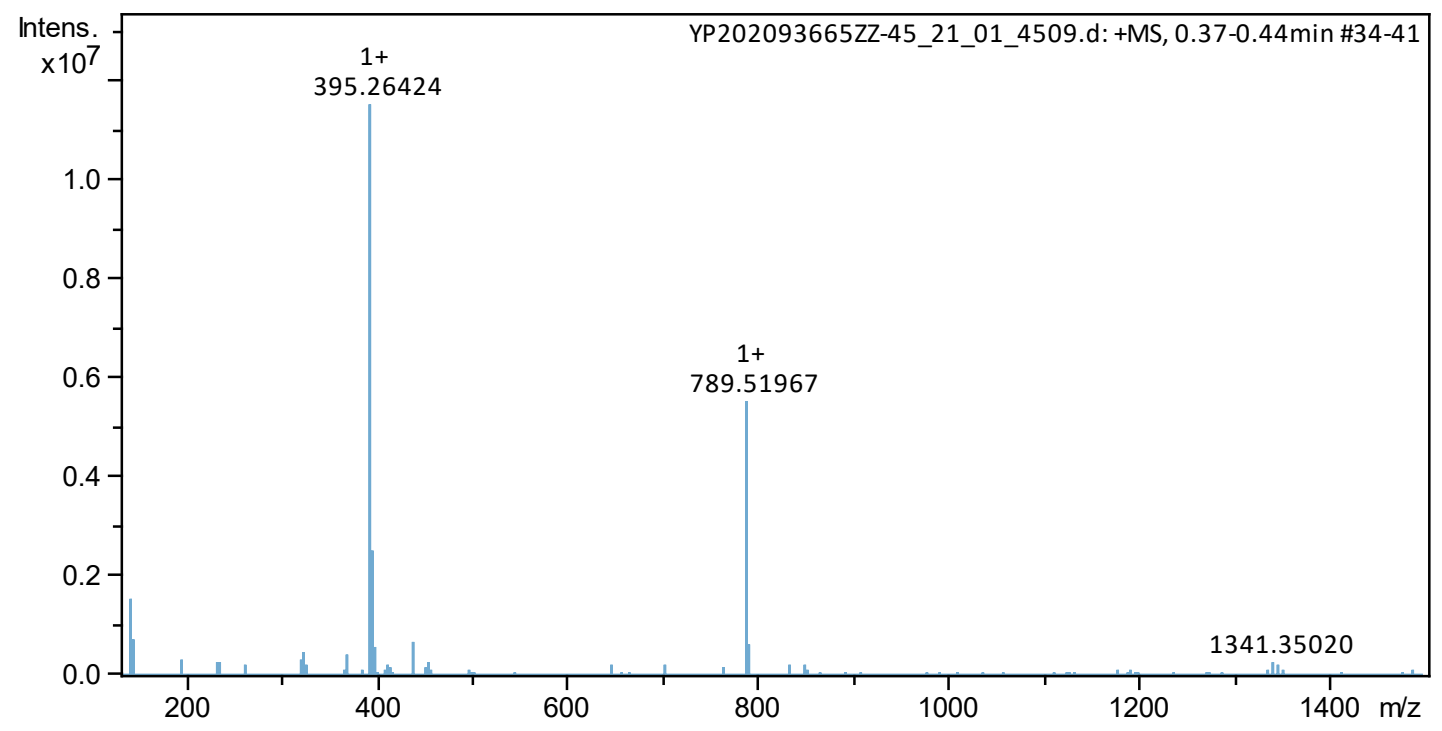

Figure S6. Electrospray ionization mass spectrum of compound 12. 


\section{Synthesis of compound 13}

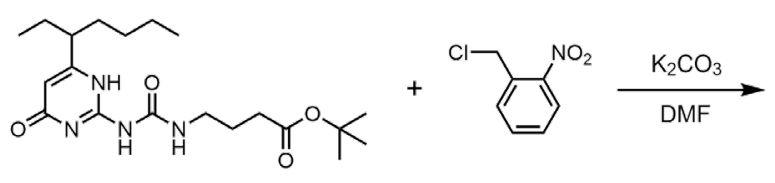

12

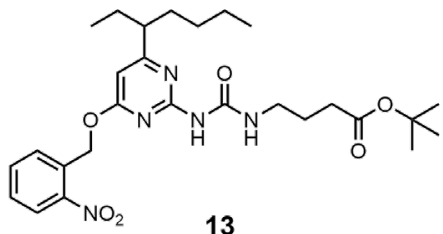

13

Compound 12 (5.30 g, $13.4 \mathrm{mmol})$ was dissolved in DMF $(80 \mathrm{~mL})$ and then nitrogen was bubbled trough for 15 minutes. Subsequently, 1-(chloromethyl)-2-nitrobenzene (3.69 g, 21.5 mmol $)$ and $\mathrm{K}_{2} \mathrm{CO}_{3}(2.96 \mathrm{~g}, 21.5 \mathrm{mmol})$ were added and the mixture was stirred overnight at 80 ${ }^{\circ} \mathrm{C}$ under $\mathrm{N}_{2}$. The solvent was evaporated, the residue suspended in a mixture of chloroform and water, the layers were separated and the aqueous phase was extracted with chloroform. The organic phase was dried using $\mathrm{Na}_{2} \mathrm{SO}_{4}$ and the solvent was removed under vacuum. The crude was further purified by gel chromatography to afford compound $\mathbf{1 3}$ as an orange-yellow oil (5.26 g, 74\%). The ${ }^{1} \mathrm{H}$ NMR spectrum of monomer 13 is shown in Figure $\mathrm{S} 7 .{ }^{1} \mathrm{H}$ NMR $\left(\mathrm{CDCl}_{3}\right.$, room temperature, $400 \mathrm{MHz}) \delta(\mathrm{ppm}): 9.31(\mathrm{~s}, 1 \mathrm{H}), 8.15(\mathrm{dd}, J=8.0,1.2 \mathrm{~Hz}, 1 \mathrm{H}), 7.75-7.62$ (m, 2H), 7.56-7.48 (m, 1H), $7.11(\mathrm{~s}, 1 \mathrm{H}), 6.25(\mathrm{~s}, 1 \mathrm{H}), 5.76(\mathrm{~s}, 2 \mathrm{H}), 3.45-3.35(\mathrm{~m}, 2 \mathrm{H})$, 2.55-2.41 (m, 1H), 2.33 (t, $J=7.6 \mathrm{~Hz}, 2 \mathrm{H}), 1.94-1.84(\mathrm{~m}, 2 \mathrm{H}), 1.73-1.55(\mathrm{~m}, 4 \mathrm{H}), 1.45(\mathrm{~s}$, $9 \mathrm{H}), 1.37-1.25(\mathrm{~m}, 4 \mathrm{H}), 0.92-0.80(\mathrm{~m}, 6 \mathrm{H})$. The ${ }^{13} \mathrm{C}$ NMR spectrum of compound $\mathbf{1 3}$ is shown in Figure $\mathrm{S} 8 .{ }^{13} \mathrm{C}$ NMR $\left(\mathrm{CDCl}_{3}\right.$, room temperature, $\left.100 \mathrm{MHz}\right) \delta(\mathrm{ppm}): 172.32,169.50,157.45$, $147.71,133.67,132.37,129.19,128.73,124.98,100.31,80.27,64.72,60.38,48.89,39.24$, $34.24,32.99,31.50,31.43,30.19,30.13,29.69,29.65,29.59,28.07,27.77,25.42,22.72,22.68$, 21.03, 14.19, 14.10, 13.95. HRESIMS is shown in Figure S9: $m / z$ calcd for $\mathrm{C}_{27} \mathrm{H}_{39} \mathrm{~N}_{5} \mathrm{O}_{6}$, $530.29731[\mathrm{M}+\mathrm{H}]^{+}$; found $530.29542[\mathrm{M}+\mathrm{H}]^{+}$. 


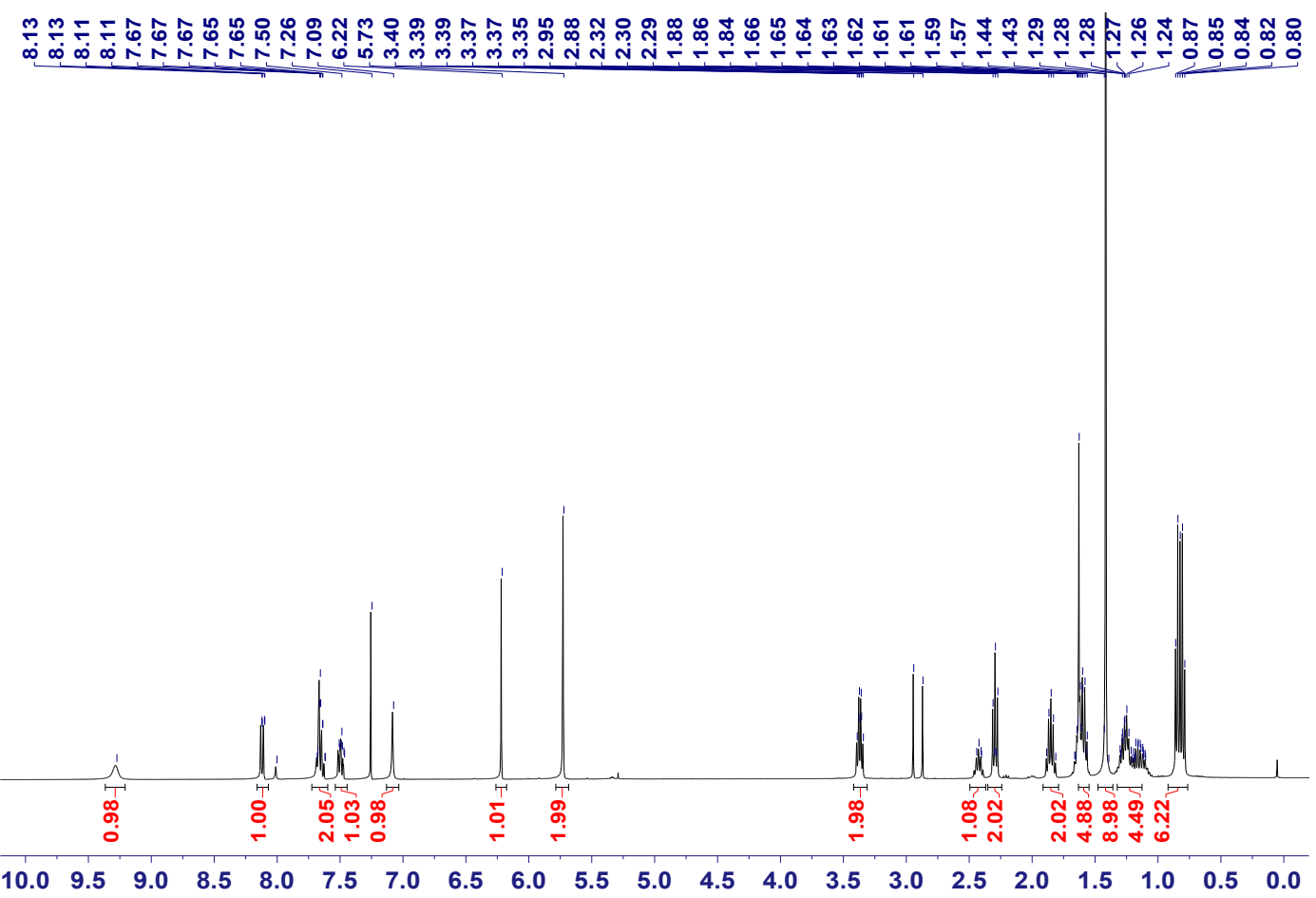

Figure S7. ${ }^{1} \mathrm{H}$ NMR spectrum $\left(\mathrm{CDCl}_{3}\right.$, room temperature, $\left.400 \mathrm{MHz}\right)$ of compound 13.
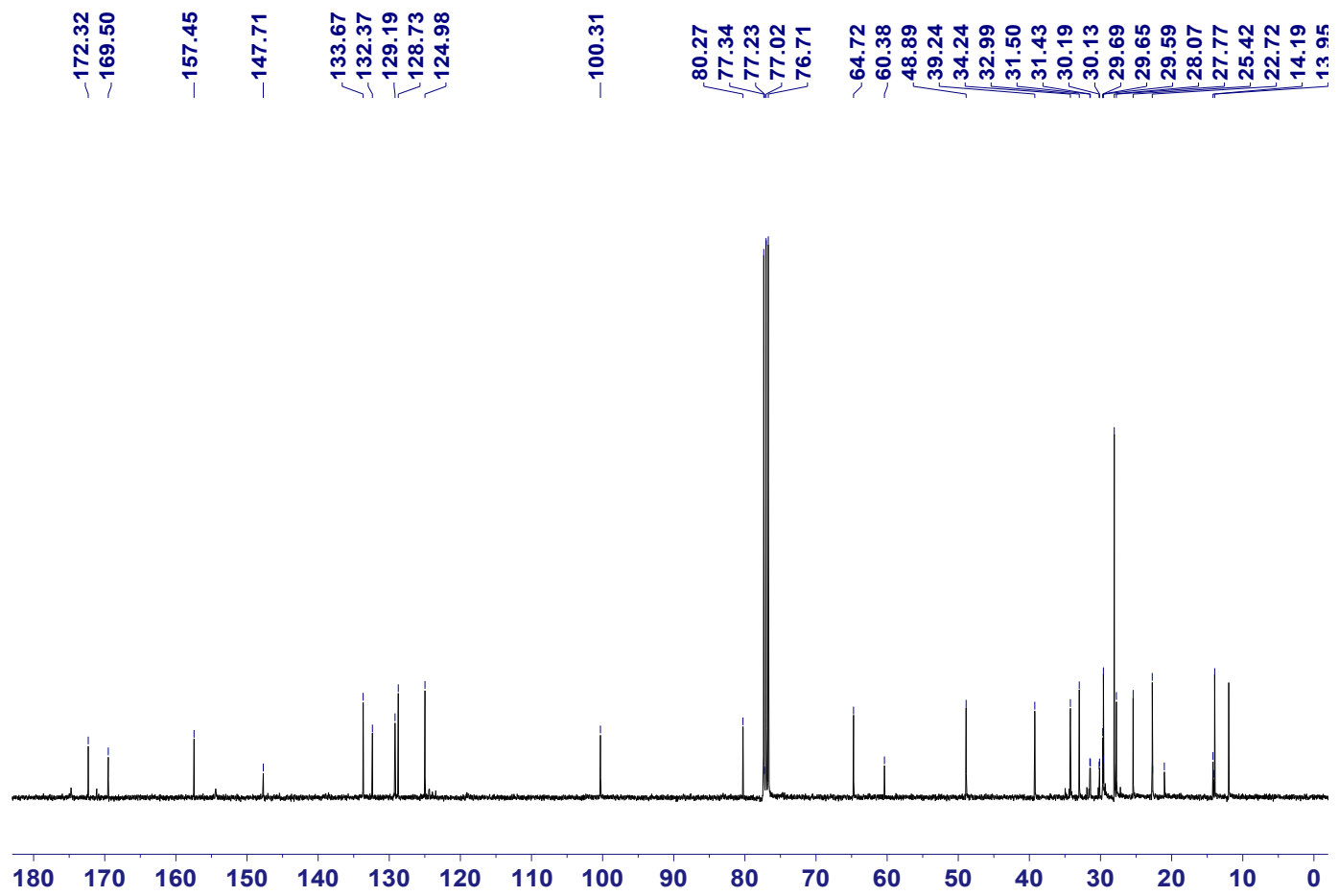

Figure S8. ${ }^{13} \mathrm{C}$ NMR spectrum $\left(\mathrm{CDCl}_{3}\right.$, room temperature, $\left.100 \mathrm{MHz}\right)$ of compound 13. 


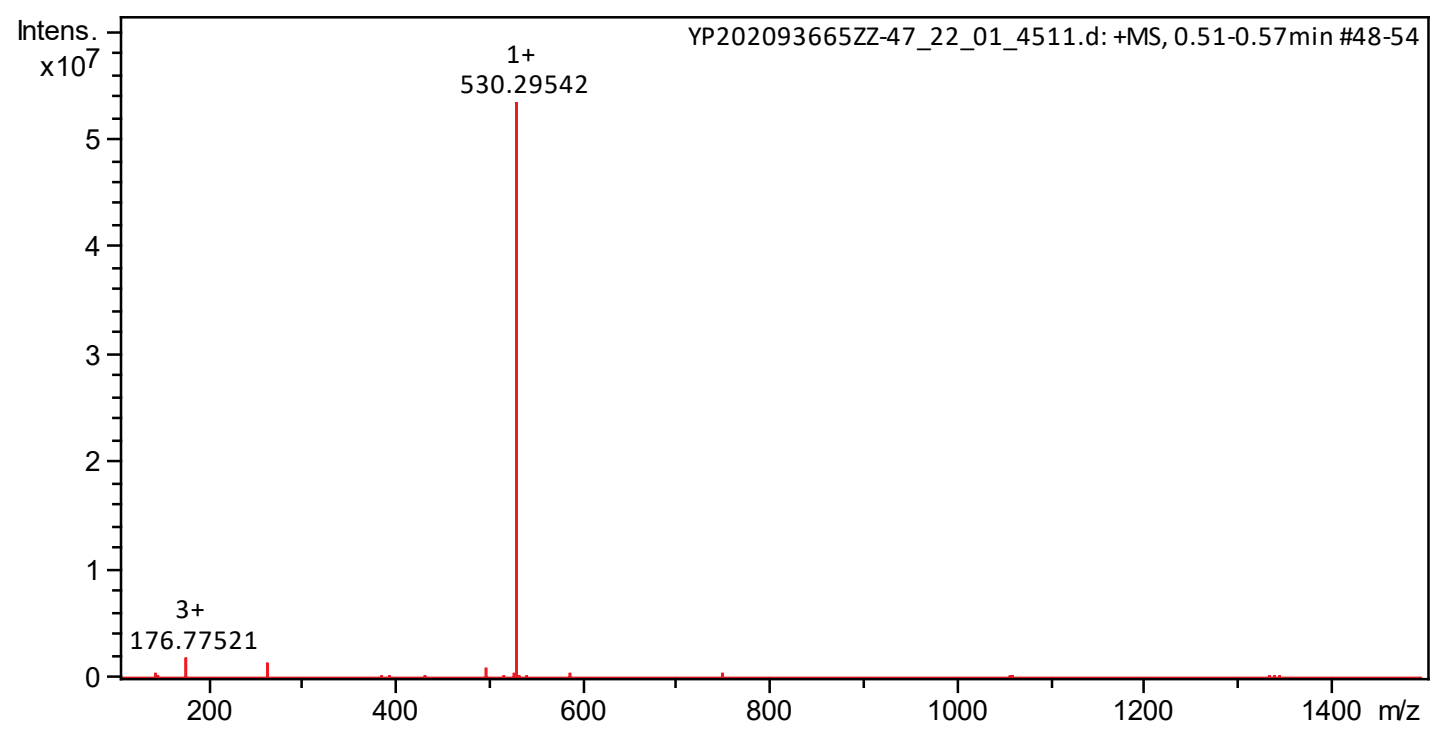

Figure S9. Electrospray ionization mass spectrum of compound 13. 


\section{Synthesis of compound 14}

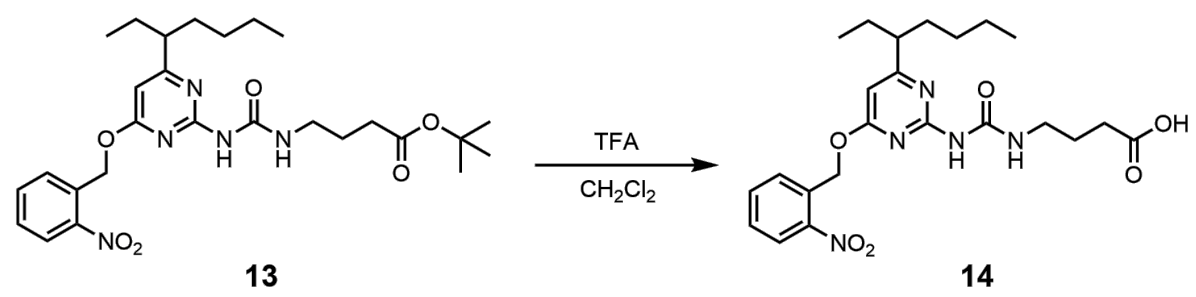

Compound 13 (3.30 g, $4.07 \mathrm{mmol})$ was dissolved in a mixture of DCM (70 mL) and TFA (3.50 $\mathrm{mL}$ ). The mixture was stirred overnight at room temperature under $\mathrm{N}_{2}$. The solvent was evaporated and the crude product was purified using column chromatography to yield compound 14 as an orange-yellow oil (2.23 g, 54\%). The ${ }^{1} \mathrm{H}$ NMR spectrum of compound 14 is shown in Figure $\mathrm{S} 10 .{ }^{1} \mathrm{H}$ NMR $\left(\mathrm{CDCl}_{3}\right.$, room temperature, $\left.400 \mathrm{MHz}\right) \delta(\mathrm{ppm}): 9.45(\mathrm{~s}, 1 \mathrm{H})$, $8.09(\mathrm{~d}, J=8.0 \mathrm{~Hz}, 1 \mathrm{H}), 7.97-7.87(\mathrm{~m}, 1 \mathrm{H}), 7.68(\mathrm{t}, J=7.6 \mathrm{~Hz}, 1 \mathrm{H}), 7.53(\mathrm{t}, J=7.6 \mathrm{~Hz}, 1 \mathrm{H})$, $6.30(\mathrm{~s}, 1 \mathrm{H}), 5.86(\mathrm{~s}, 2 \mathrm{H}), 3.47-3.32(\mathrm{~m}, 2 \mathrm{H}), 2.60-2.48(\mathrm{~m}, 1 \mathrm{H}), 2.44(\mathrm{t}, J=7.2 \mathrm{~Hz}, 2 \mathrm{H})$, 2.00-1.87 (m, 2H), 1.82-1.49 (m, 4H), 1.39-1.07 (m, 5H), $0.86(\mathrm{t}, J=7.2 \mathrm{~Hz}, 6 \mathrm{H})$. The ${ }^{13} \mathrm{C}$ NMR spectrum of compound 14 is shown in Figure $\mathrm{S} 11 .{ }^{13} \mathrm{C} \mathrm{NMR}\left(\mathrm{CDCl}_{3}\right.$, room temperature, $100 \mathrm{MHz}) \delta$ (ppm): 177.29, 171.29, 162.40, 156.15, 148.04, 133.93, 129.72, 125.03, 101.09, $39.29,33.57,31.43,29.34,29.30,27.26,24.73,22.52,13.80,11.72$. HRESIMS is shown in Figure $\mathrm{S} 12: \mathrm{m} / z$ calcd for $\mathrm{C}_{23} \mathrm{H}_{31} \mathrm{~N}_{5} \mathrm{O}_{6}, 474.23471[\mathrm{M}+\mathrm{H}]^{+}$; found $474.23314[\mathrm{M}+\mathrm{H}]^{+}$. 


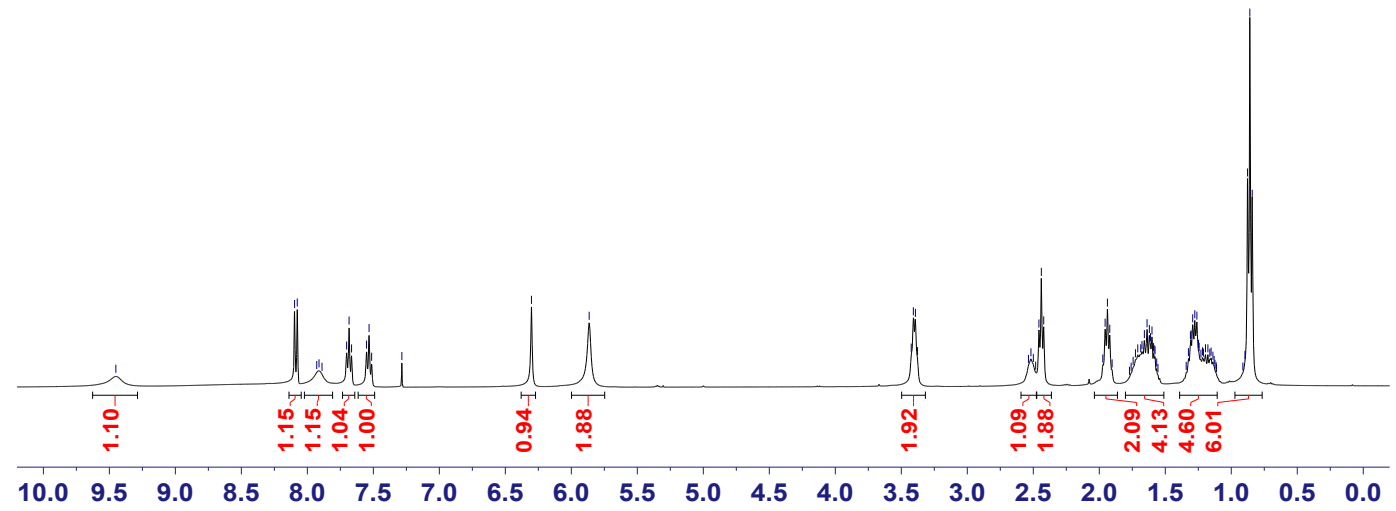

Figure S10. ${ }^{1} \mathrm{H}$ NMR spectrum $\left(\mathrm{CDCl}_{3}\right.$, room temperature, $\left.400 \mathrm{MHz}\right)$ of compound 14.

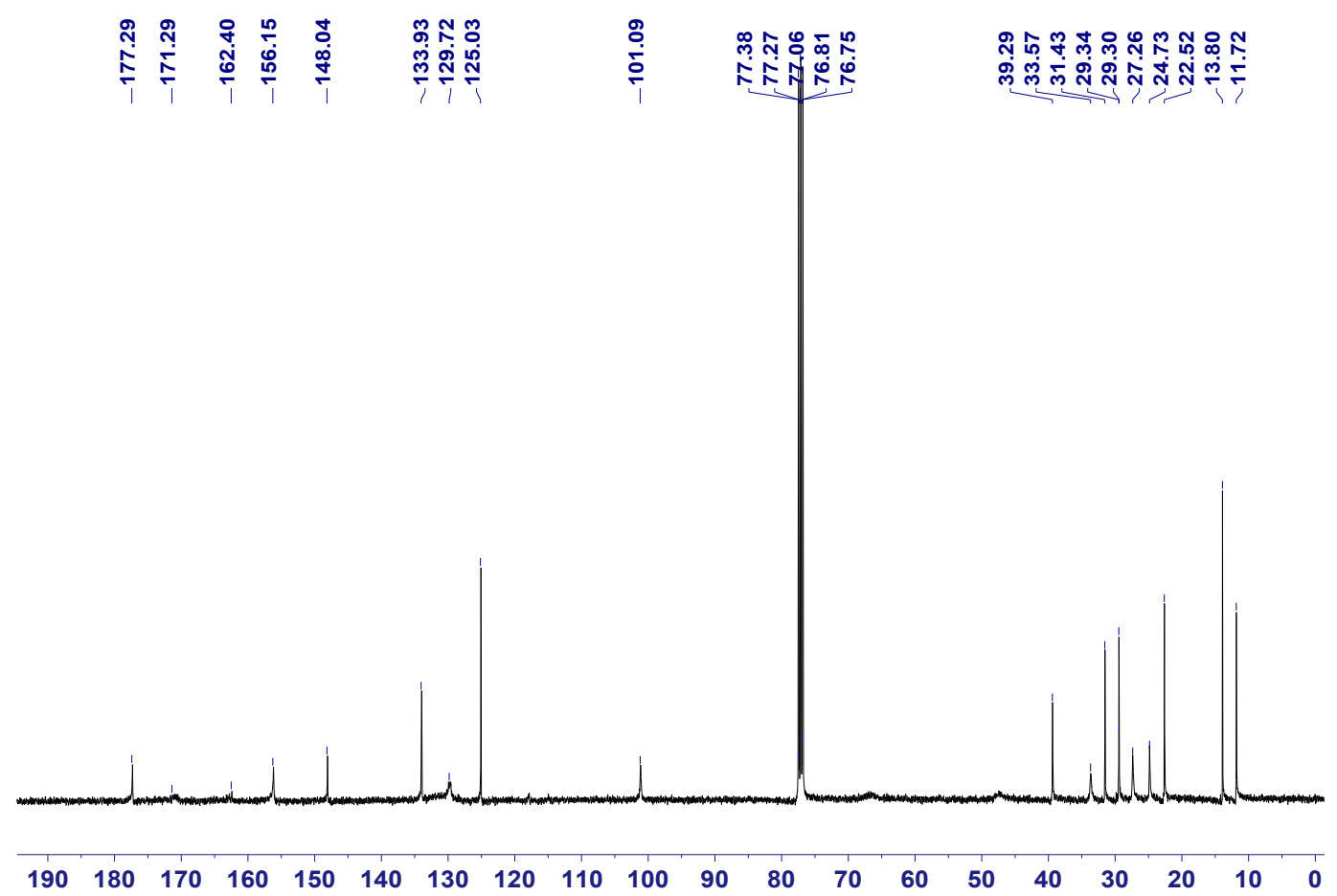

Figure S11. ${ }^{13} \mathrm{C}$ NMR spectrum $\left(\mathrm{CDCl}_{3}\right.$, room temperature, $\left.100 \mathrm{MHz}\right)$ of compound 14. 


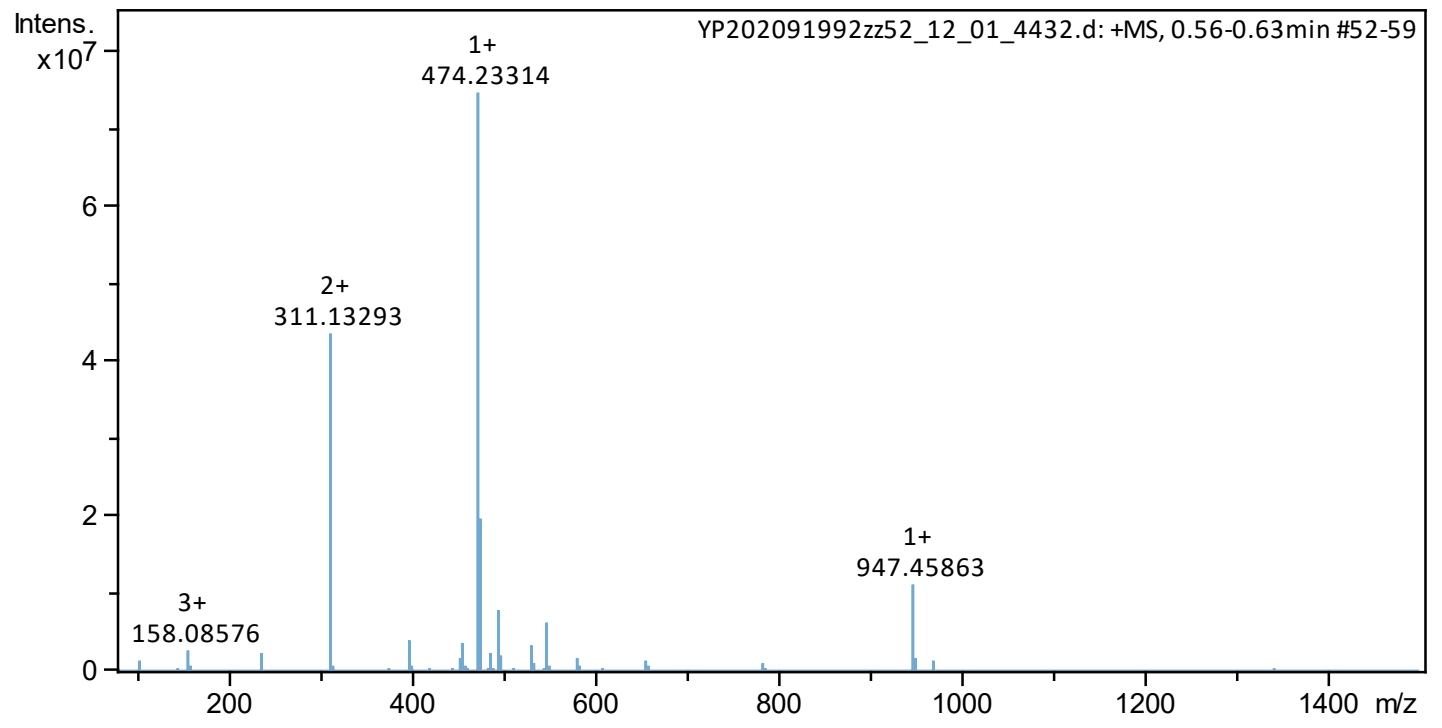

Figure S12. Electrospray ionization mass spectrum of compound 14. 


\section{Synthesis of compound 18}

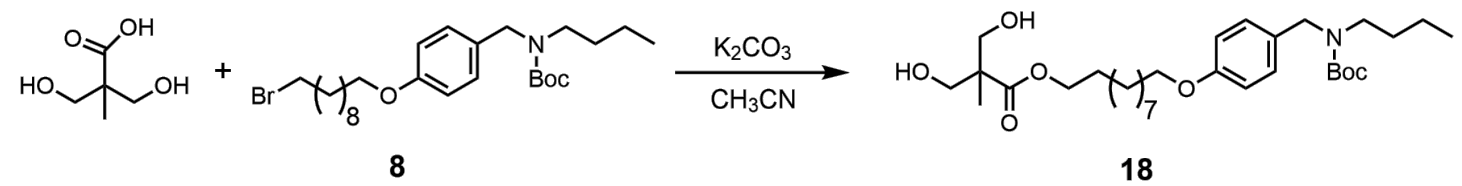

A vial was charged with 2,2-bis(hydroxymethyl)propionic acid (0.80 g, $6.00 \mathrm{mmol})$, compound $8(2.50 \mathrm{~g}, 5.01 \mathrm{mmol})$ and $\mathrm{K}_{2} \mathrm{CO}_{3}(1.66 \mathrm{~g}, 12.0 \mathrm{mmol})$. The vial was degassed, and then $40 \mathrm{~mL}$ of acetonitrile was added via syringe under a nitrogen atmosphere. The reaction was heated to reflux for $12 \mathrm{~h}$. The reaction mixture was cooled to filter salts out and the filtrate was evaporated under vacuum. The crude was dissolved in DCM and washed by water and saturated aqueous sodium chloride. The organic phase was dried using $\mathrm{Na}_{2} \mathrm{SO}_{4}$ and the crude was further purified by gel chromatography to afford compound 18 as a colorless oil $(1.19 \mathrm{~g}, 43 \%)$. The ${ }^{1} \mathrm{H}$ NMR spectrum of compound 18 is shown in Figure $\mathrm{S} 13 .{ }^{1} \mathrm{H}$ NMR $\left(\mathrm{CDCl}_{3}\right.$, room temperature, 400 MHz) $\delta(\mathrm{ppm}): 7.16(\mathrm{~s}, 2 \mathrm{H}), 6.91-6.80(\mathrm{~m}, 2 \mathrm{H}), 4.37$ (s, 2H), $4.18(\mathrm{t}, J=6.8 \mathrm{~Hz}, 2 \mathrm{H}), 4.00-3.88$ (m, 4H), 3.80-3.68 (m, 2H), 3.26-3.04 (m, 2H), $2.88(\mathrm{t}, J=6.4 \mathrm{~Hz}, 2 \mathrm{H}), 1.85-1.74(\mathrm{~m}, 2 \mathrm{H})$, $1.68(\mathrm{~d}, J=6.0 \mathrm{~Hz}, 4 \mathrm{H}), 1.37-1.26(\mathrm{~m}, 13 \mathrm{H}), 1.41-1.21(\mathrm{~m}, 10 \mathrm{H}), 1.08(\mathrm{~s}, 3 \mathrm{H}), 0.91(\mathrm{t}, J=7.2$ $\mathrm{Hz}, 3 \mathrm{H})$. The ${ }^{13} \mathrm{C}$ NMR spectrum of compound 18 is shown in Figure $\mathrm{S} 14 .{ }^{13} \mathrm{C}$ NMR $\left(\mathrm{CDCl}_{3}\right.$, room temperature, $100 \mathrm{MHz}) \delta(\mathrm{ppm}): 176.00,158.26,130.47,128.97,128.40,114.39,79.41$, $68.16,67.98,65.16,49.70,49.11,46.05,30.14,29.44,29.39,29.33,29.27,29.16,28.51,28.48$, 26.03, 25.83, 20.02, 17.17, 13.86. HRESIMS is shown in Figure S15: $\mathrm{m} / z$ calcd for $\mathrm{C}_{31} \mathrm{H}_{53} \mathrm{NO}_{7}$, $552.38948[\mathrm{M}+\mathrm{H}]^{+}$; found $552.38714[\mathrm{M}+\mathrm{H}]^{+}$. 


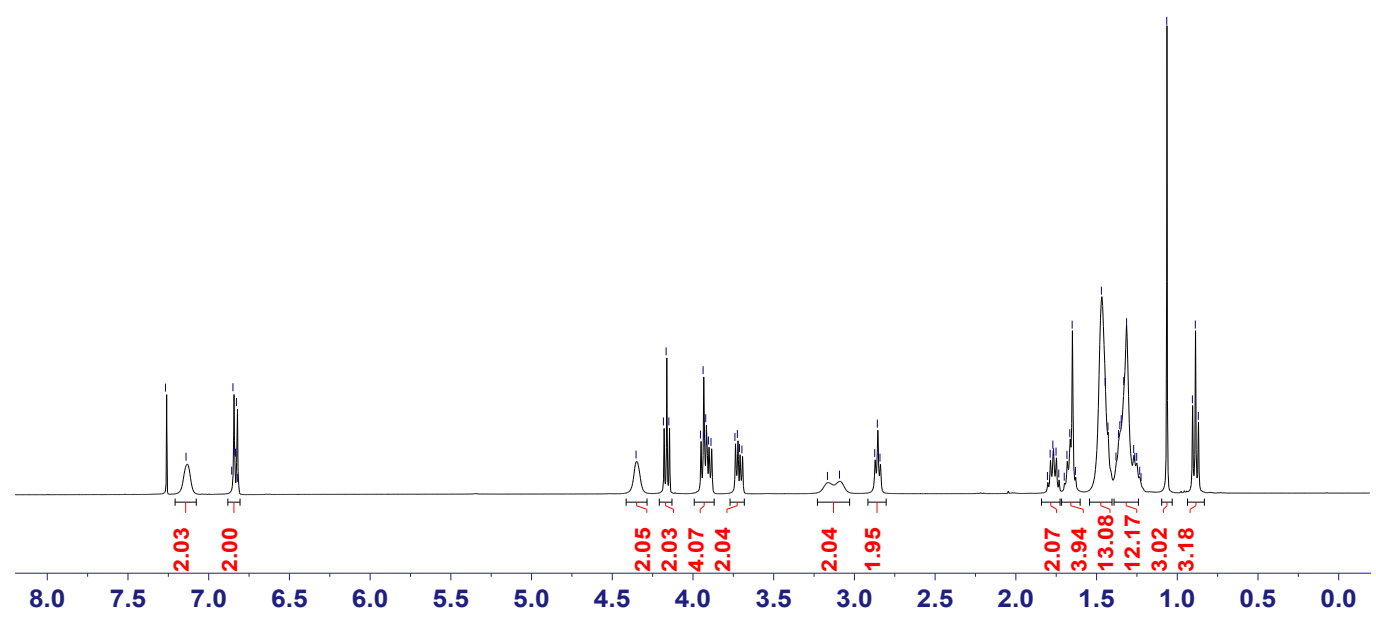

Figure S13. ${ }^{1} \mathrm{H}$ NMR spectrum $\left(\mathrm{CDCl}_{3}\right.$, room temperature, $\left.400 \mathrm{MHz}\right)$ of compound 18.

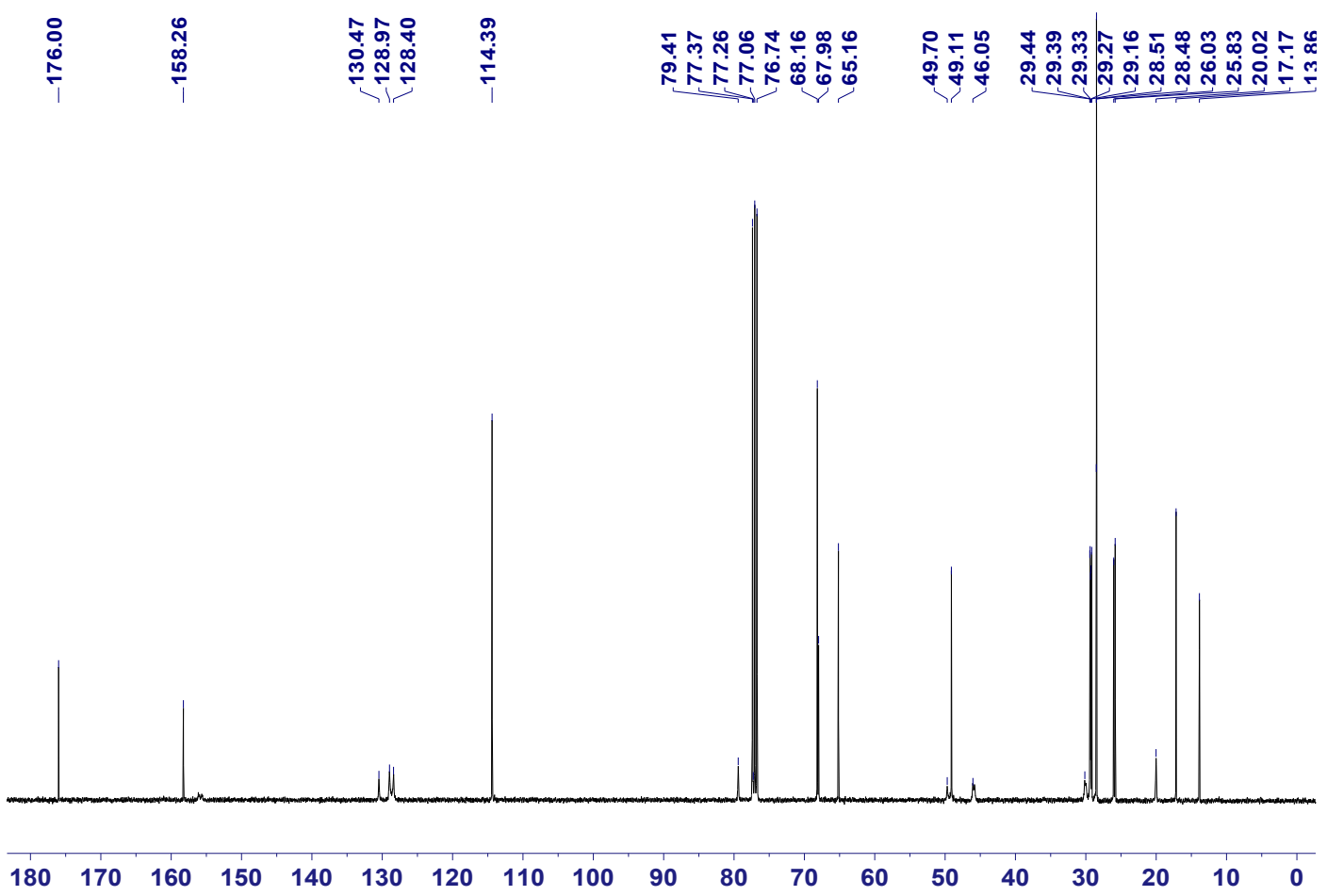

Figure S14. ${ }^{13} \mathrm{C}$ NMR spectrum $\left(\mathrm{CDCl}_{3}\right.$, room temperature, $\left.100 \mathrm{MHz}\right)$ of compound 18 . 


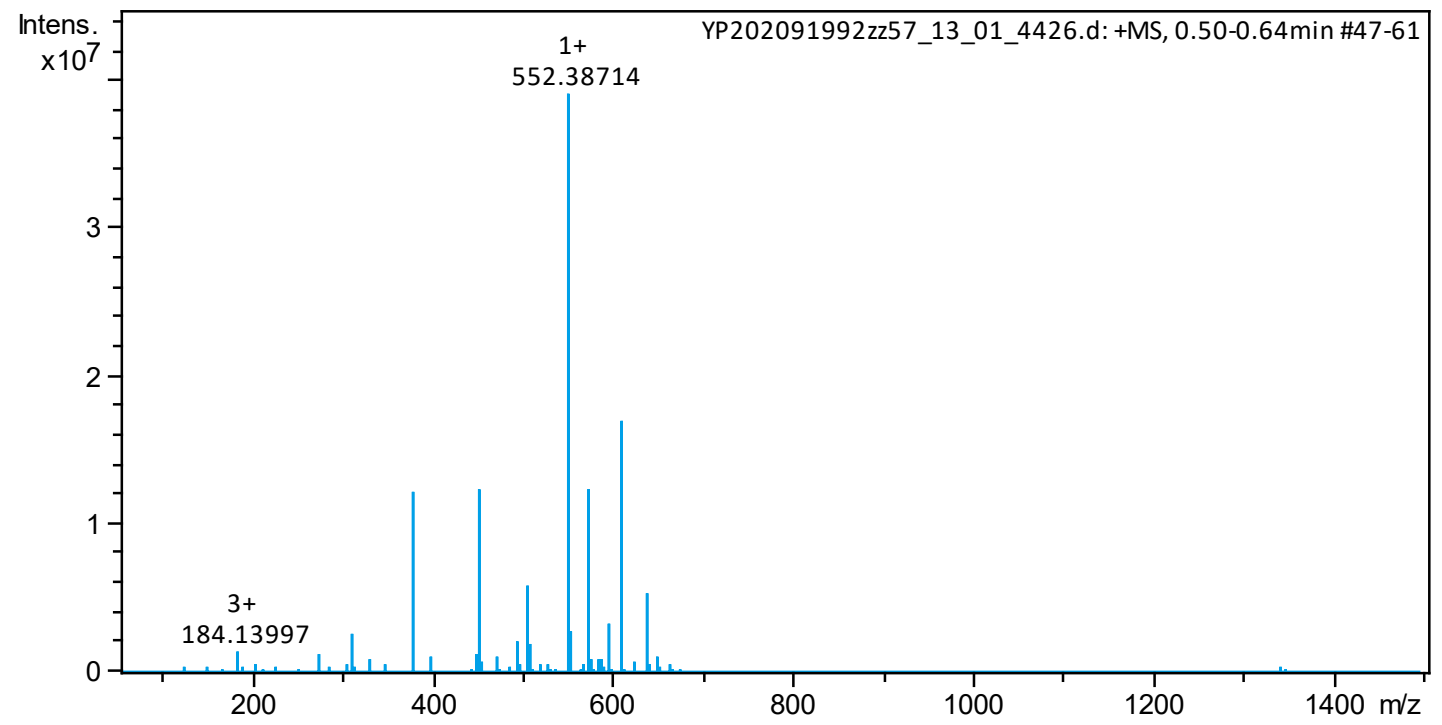

Figure S15. Electrospray ionization mass spectrum of compound 18. 


\section{Synthesis of compound 19}

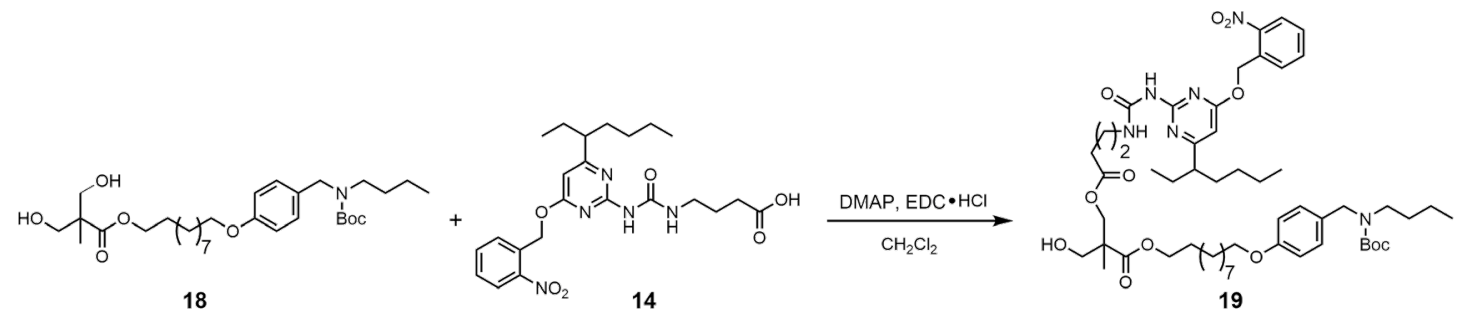

Compound 18 (3.71 g, $6.72 \mathrm{mmol})$, EDC $\cdot \mathrm{HCl}(1.29 \mathrm{~g}, 6.73 \mathrm{mmol})$, and DMAP (247 mg, 2.02 mmol) were dissolved in $150 \mathrm{~mL}$ DCM. Compound 14 (3.20 g, $6.73 \mathrm{mmol})$ was added. The mixture was stirred at room temperature overnight and purified via gel chromatography to afford compound 19 as a light yellow oil (5.88 g, 87\%). The ${ }^{1} \mathrm{H}$ NMR spectrum of compound 19 is shown in Figure $\mathrm{S} 16 .{ }^{1} \mathrm{H}$ NMR $\left(\mathrm{CDCl}_{3}\right.$, room temperature, $\left.400 \mathrm{MHz}\right) \delta(\mathrm{ppm}): 9.32$ (s, $1 \mathrm{H}), 8.12(\mathrm{dd}, J=8.0,1.2 \mathrm{~Hz}, 1 \mathrm{H}), 7.73-7.61(\mathrm{~m}, 2 \mathrm{H}), 7.54-7.46(\mathrm{~m}, 1 \mathrm{H}), 7.20-7.10(\mathrm{~m}, 3 \mathrm{H})$, 6.92-6.77 (m, 2H), $6.24(\mathrm{~s}, 1 \mathrm{H}), 5.73(\mathrm{~s}, 2 \mathrm{H}), 4.39-4.26(\mathrm{~m}, 3 \mathrm{H}), 4.19(\mathrm{~d}, J=11.2 \mathrm{~Hz}, 1 \mathrm{H})$, $4.10(\mathrm{t}, J=6.8 \mathrm{~Hz}, 2 \mathrm{H}), 3.92(\mathrm{t}, J=6.8 \mathrm{~Hz}, 2 \mathrm{H}), 3.76-3.61(\mathrm{~m}, 2 \mathrm{H}), 3.45-3.32(\mathrm{~m}, 2 \mathrm{H})$, 3.22-3.01 (m, 2H), 2.49-2.37 (m, 3H), 1.93-1.84 (m, 2H), 1.80-1.69 (m, 4H), 1.68-1.54 (m, $6 \mathrm{H}), 1.50-1.39$ (m, 13H), 1.37-1.25 (m, 15H), 1.18 (s, 3H), 0.93-0.78 (m, 9H). The ${ }^{13} \mathrm{C}$ NMR spectrum of compound 19 is shown in Figure $\mathrm{S} 17 .{ }^{13} \mathrm{C}$ NMR $\left(\mathrm{CDCl}_{3}\right.$, room temperature, 100 MHz) $\delta(\mathrm{ppm}): 174.48,173.02,169.50,158.28,157.35,154.60,147.64,133.75,132.32,129.09$, $128.77,125.03,114.39,100.43,79.37,68.00,65.59,65.11,64.80,64.51,48.85,48.00,38.82$, $34.21,31.32,29.57,29.48,29.43,29.37,29.30,29.18,28.50,28.48,27.76,26.05,25.82,25.48$, $22.73,20.01,17.74,13.96,13.85,11.97$. HRESIMS is shown in Figure S18: $\mathrm{m} / \mathrm{z}$ calcd for $\mathrm{C}_{54} \mathrm{H}_{82} \mathrm{~N}_{6} \mathrm{O}_{12}, 1007.60006[\mathrm{M}+\mathrm{H}]^{+}$; found $1007.60635[\mathrm{M}+\mathrm{H}]^{+}$. 


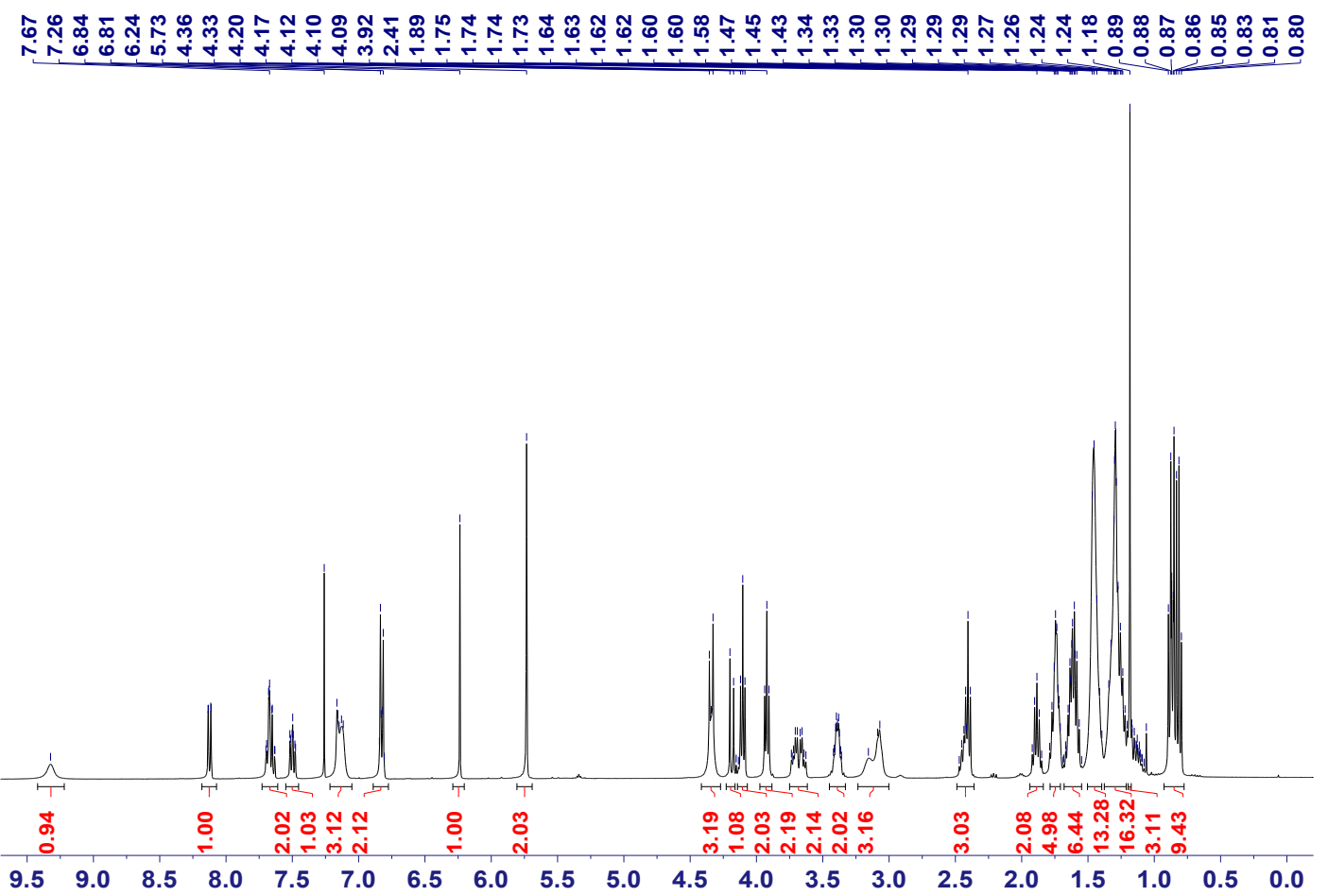

Figure S16. ${ }^{1} \mathrm{H}$ NMR spectrum $\left(\mathrm{CDCl}_{3}\right.$, room temperature, $\left.400 \mathrm{MHz}\right)$ of compound 19.

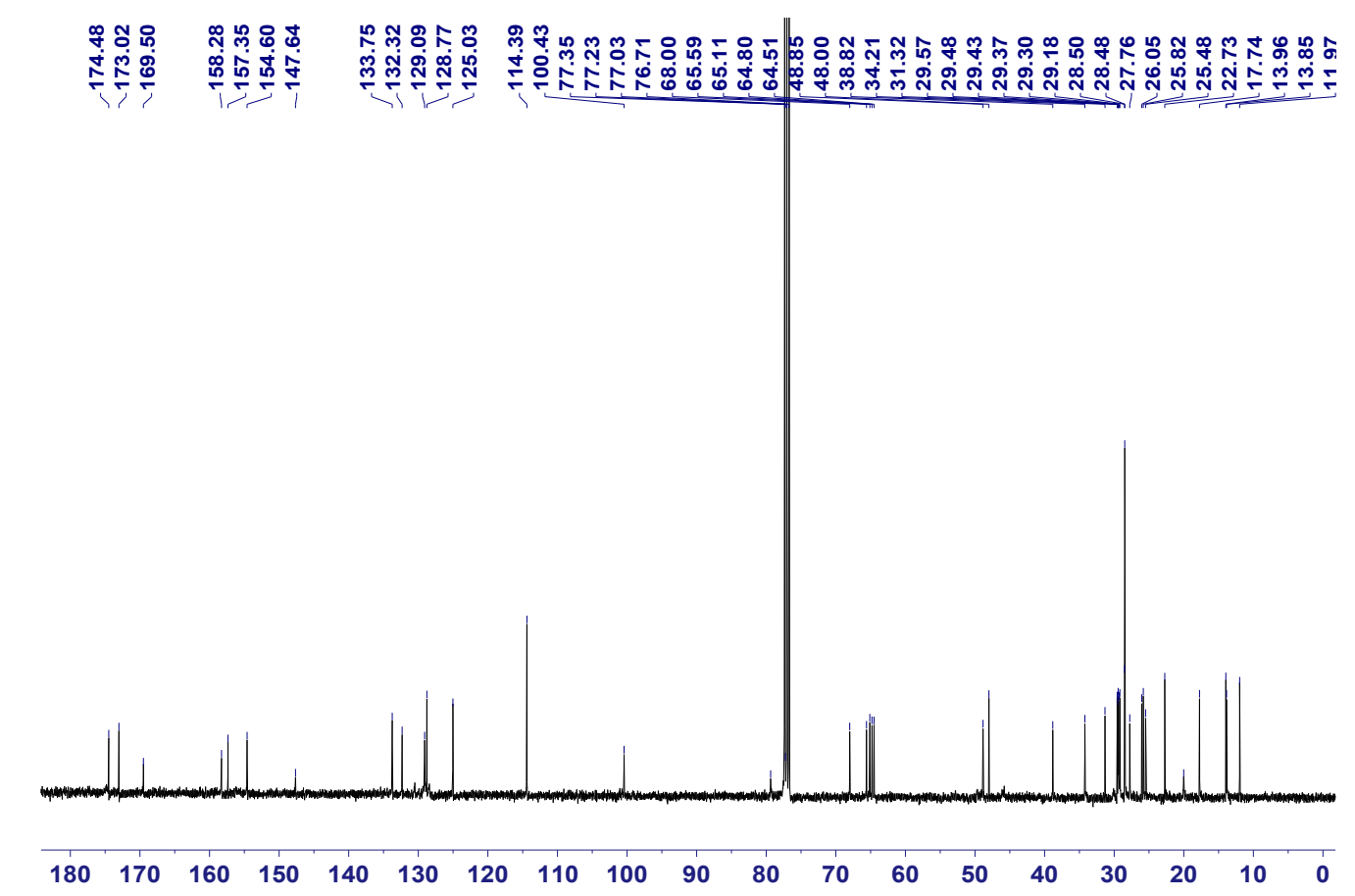

Figure S17. ${ }^{13} \mathrm{C}$ NMR spectrum $\left(\mathrm{CDCl}_{3}\right.$, room temperature, $\left.100 \mathrm{MHz}\right)$ of compound 19. 


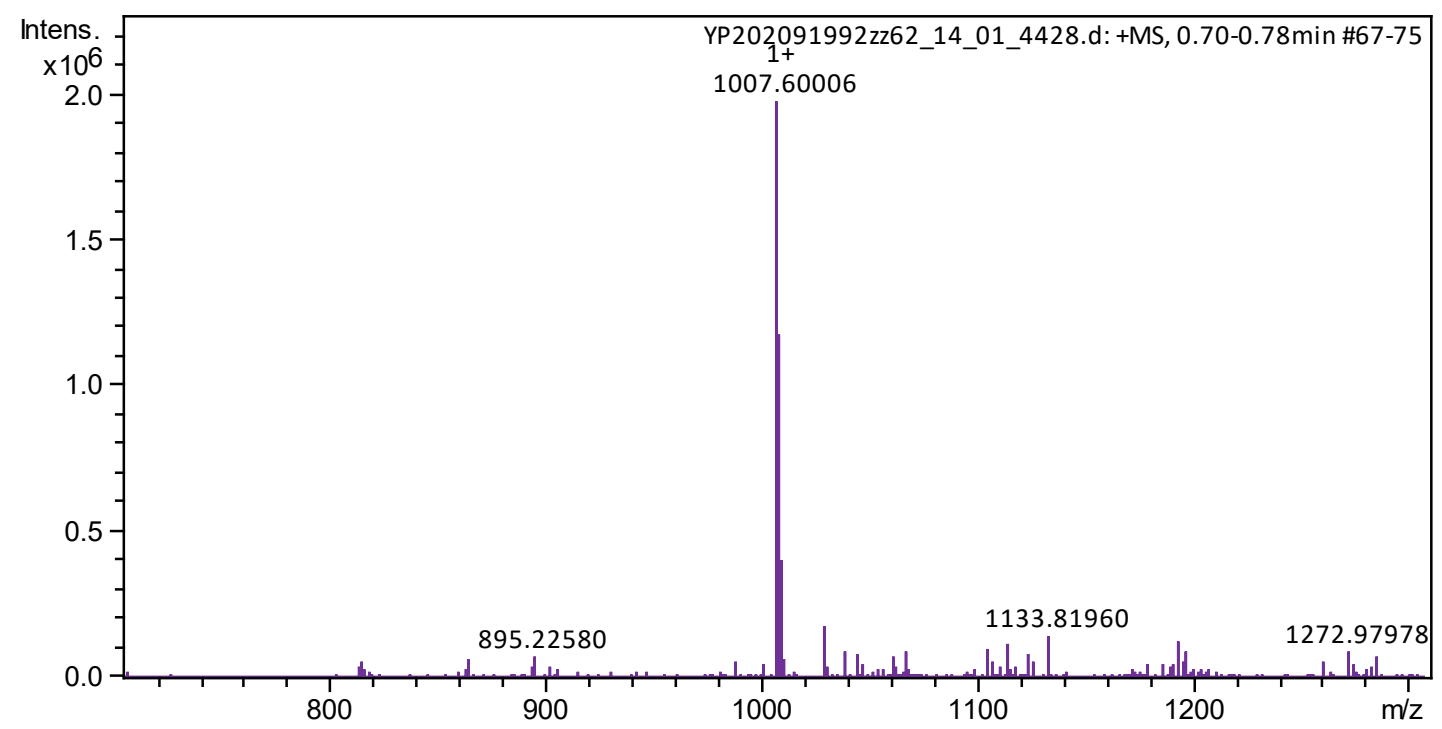

Figure S18. Electrospray ionization mass spectrum of compound 19. 


\section{Synthesis of compound 20}

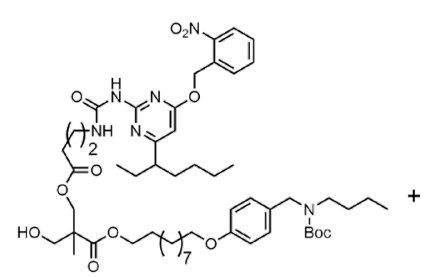

19

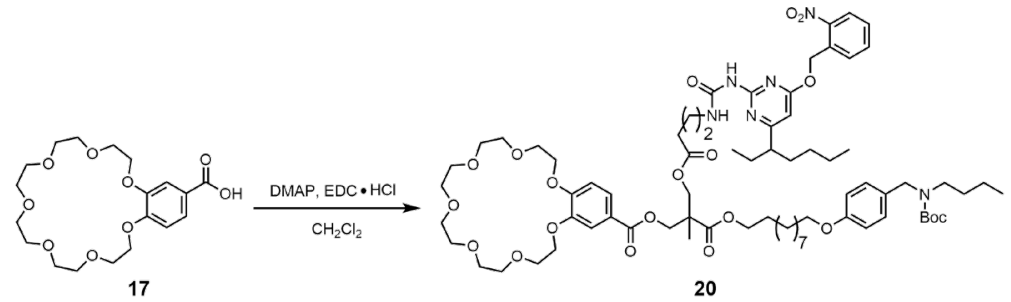

Compound 19 (5.30 g, $5.26 \mathrm{mmol}), \mathrm{EDC} \cdot \mathrm{HCl}(1.52 \mathrm{~g}, 7.93 \mathrm{mmol})$, and DMAP (326 mg, 2.60 mmol) were dissolved in $100 \mathrm{~mL}$ DCM. Compound 17 (3.20 g, $8.00 \mathrm{mmol})$ was added. The mixture was stirred at room temperature overnight and purified via gel chromatography to afford compound 20 as a light yellow oil (4.31 g, 59\%). The ${ }^{1} \mathrm{H}$ NMR spectrum of compound 20 is shown in Figure $\mathrm{S} 19 .{ }^{1} \mathrm{H}$ NMR $\left(\mathrm{CDCl}_{3}\right.$, room temperature, $\left.400 \mathrm{MHz}\right) \delta(\mathrm{ppm}): 9.28$ (s, $1 \mathrm{H}), 8.11(\mathrm{dd}, J=8.4,1.2 \mathrm{~Hz}, 1 \mathrm{H}), 7.71-7.62(\mathrm{~m}, 2 \mathrm{H}), 7.57(\mathrm{dd}, J=8.4,2.0 \mathrm{~Hz}, 1 \mathrm{H}), 7.53-$ $7.45(\mathrm{~m}, 2 \mathrm{H}), 7.11(\mathrm{~s}, 3 \mathrm{H}), 6.90-6.74(\mathrm{~m}, 3 \mathrm{H}), 6.22(\mathrm{~s}, 1 \mathrm{H}), 5.73(\mathrm{~s}, 2 \mathrm{H}), 4.41$ (d, J=1.2 Hz, 2H), 4.37-4.28 (m, 4H), 4.23-4.15 (m, 4H), $4.11(\mathrm{t}, J=6.8 \mathrm{~Hz}, 2 \mathrm{H}), 3.98-3.88(\mathrm{~m}, 6 \mathrm{H})$, $3.83-3.76(\mathrm{~m}, 4 \mathrm{H}), 3.76-3.70(\mathrm{~m}, 4 \mathrm{H}), 3.67(\mathrm{~s}, 8 \mathrm{H}), 3.42-3.31(\mathrm{~m}, 2 \mathrm{H}), 3.12(\mathrm{~d}, J=3.2 \mathrm{~Hz}$, 2H), 2.48-2.37 (m, 3H), 1.80-1.70 (m, 2H), 1.78-1.69 (m, 6H), 1.65-1.55 (m, 6H), 1.51-1.37 $(\mathrm{m}, 13 \mathrm{H}), 1.34-1.06(\mathrm{~m}, 15 \mathrm{H}), 0.92-0.76(\mathrm{~m}, 9 \mathrm{H})$. The ${ }^{13} \mathrm{C}$ NMR spectrum of compound 20 is shown in Figure $\mathrm{S} 20 .{ }^{13} \mathrm{C} \mathrm{NMR}\left(\mathrm{CDCl}_{3}\right.$, room temperature, $\left.100 \mathrm{MHz}\right) \delta(\mathrm{ppm}): 172.79,172.50$, $169.48,165.64,158.27,157.38,154.32,153.10,148.32,147.63,133.73,132.36,129.08$, $128.74,125.01,123.92,122.35,114.46,114.38,112.20,100.34,71.30,71.20,71.10,70.99$, $70.97,70.55,69.61,69.45,69.24,69.08,68.00,65.87,65.60,65.38,64.75,48.85,46.53,39.11$, $34.21,31.48,29.57,29.49,29.43,29.39,29.31,29.19,28.53,28.49,27.76,26.06,25.83,25.30$, $22.72,17.90,13.97,13.86,11.98$. HRESIMS is shown in Figure S21: $\mathrm{m} / \mathrm{z}$ calcd for $\mathrm{C}_{73} \mathrm{H}_{108} \mathrm{~N}_{6} \mathrm{O}_{20}, 1406.79567\left[\mathrm{M}+\mathrm{NH}_{4}\right]^{+}$; found $1406.73021\left[\mathrm{M}+\mathrm{NH}_{4}\right]^{+}$. 


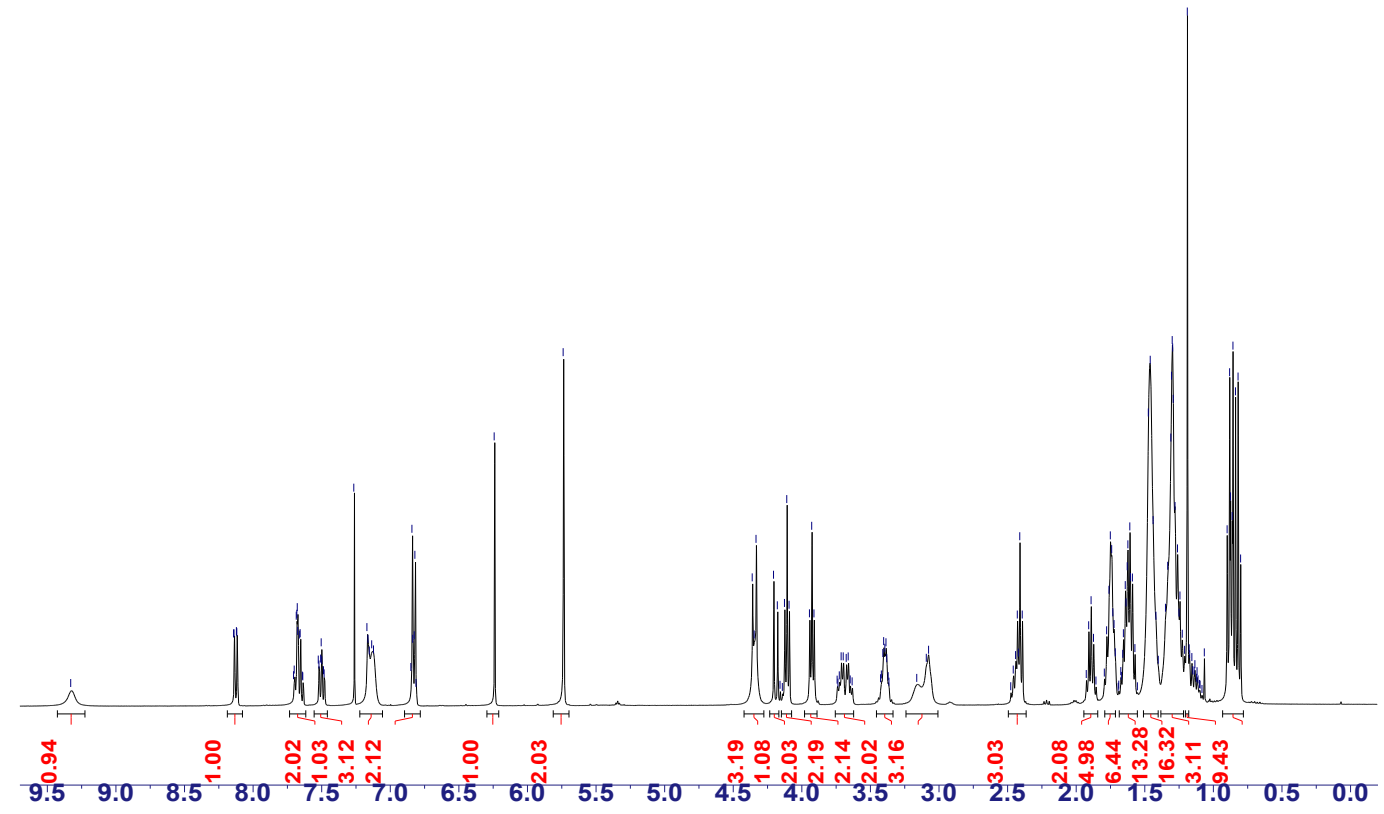

Figure S19. ${ }^{1} \mathrm{H}$ NMR spectrum $\left(\mathrm{CDCl}_{3}\right.$, room temperature, $\left.400 \mathrm{MHz}\right)$ of compound $\mathbf{2 0}$.

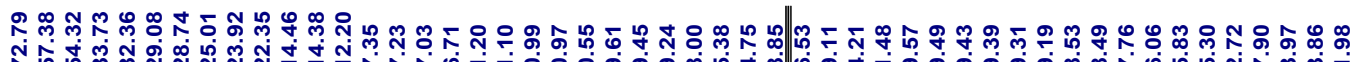

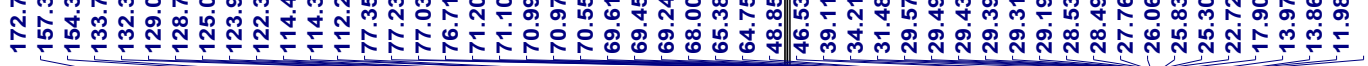

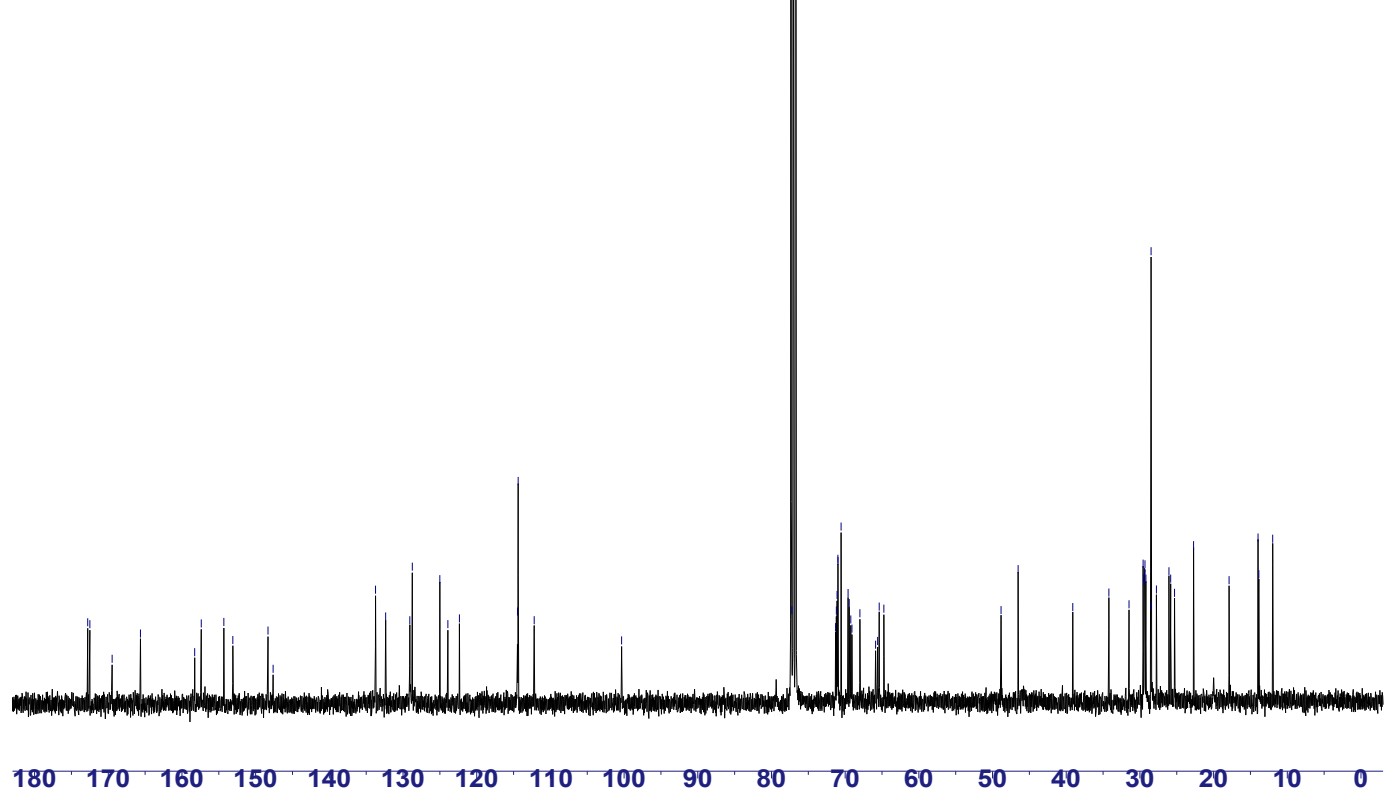

Figure S20. ${ }^{13} \mathrm{C}$ NMR spectrum $\left(\mathrm{CDCl}_{3}\right.$, room temperature, $\left.100 \mathrm{MHz}\right)$ of compound 20 . 


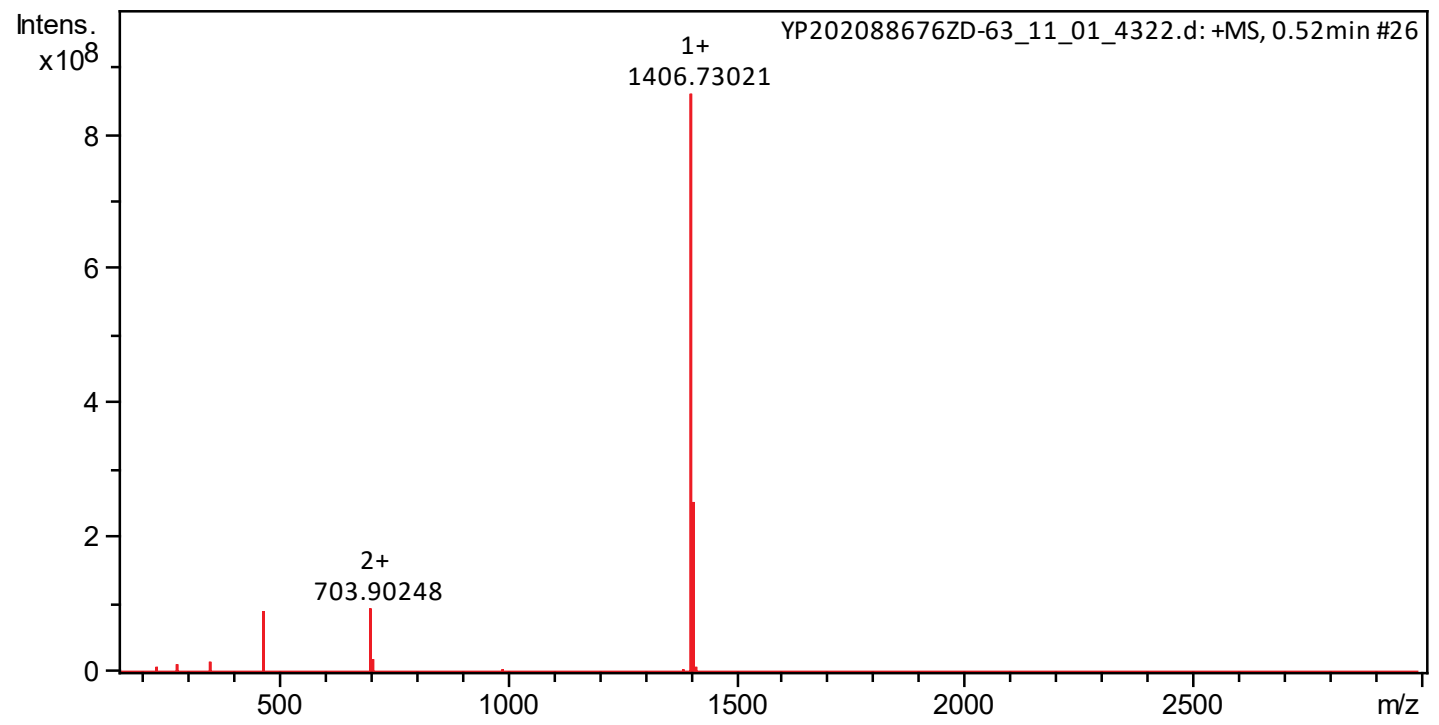

Figure S21. Electrospray ionization mass spectrum of compound 20. 


\section{Synthesis of compound 21}

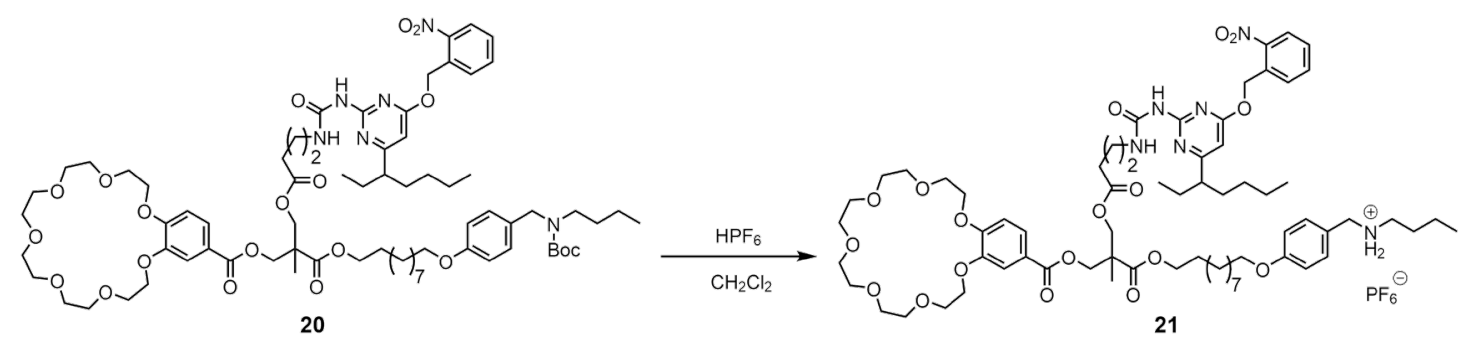

$\mathrm{HPF}_{6}(2.41 \mathrm{~g}, 14.4 \mathrm{mmol})$ was added to a solution of compound 20 (2.02 g, $\left.1.45 \mathrm{mmol}\right)$ in DCM and the mixture was stirred overnight at room temperature. The solution was washed by pure water 3 times. Then the solvent was removed by evaporation to afford compound $\mathbf{2 1}$ as light yellow solid (1.99g, 96\%). M.P. $=46{ }^{\circ} \mathrm{C}$. The ${ }^{1} \mathrm{H}$ NMR spectrum of compound 21 is shown in Figure S22. ${ }^{1} \mathrm{H}$ NMR (DMSO- $d_{6}$, room temperature, $\left.400 \mathrm{MHz}\right) \delta(\mathrm{ppm}): 9.52(\mathrm{~s}, 1 \mathrm{H}), 9.27(\mathrm{~s}$, 1H), 9.05-8.22 (m, 2H), 8.16-8.07 (m, 1H), 7.83-7.74 (m, 2H), 7.68-7.57 (m, 1H), $7.52(\mathrm{dd}$, $J=8.4,2.0 \mathrm{~Hz}, 1 \mathrm{H}), 7.43-7.33(\mathrm{~m}, 3 \mathrm{H}), 7.06$ (d, $J=8.4 \mathrm{~Hz}, 1 \mathrm{H}), 7.00-6.91(\mathrm{~m}, 2 \mathrm{H}), 6.41(\mathrm{~s}$, 1H), $5.71(\mathrm{~s}, 2 \mathrm{H}), 4.34(\mathrm{~s}, 2 \mathrm{H}), 4.26(\mathrm{~s}, 2 \mathrm{H}), 4.20-4.13(\mathrm{~m}, 2 \mathrm{H}), 4.12-4.09(\mathrm{~m}, 2 \mathrm{H}), 4.04$ (d, $J$ $=7.6 \mathrm{~Hz}, 4 \mathrm{H}), 3.93(\mathrm{t}, J=6.4 \mathrm{~Hz}, 2 \mathrm{H}), 3.81-3.72(\mathrm{~m}, 4 \mathrm{H}), 3.58-3.63(\mathrm{~m}, 4 \mathrm{H}), 3.57-3.52(\mathrm{~m}$, 4H), $3.50(\mathrm{~s}, 8 \mathrm{H}), 3.25-3.17(\mathrm{~m}, 2 \mathrm{H}), 2.90-2.81(\mathrm{~m}, 2 \mathrm{H}), 2.37(\mathrm{t}, J=7.2 \mathrm{~Hz}, 2 \mathrm{H}), 1.73(\mathrm{t}, J=$ $7.2 \mathrm{~Hz}, 2 \mathrm{H}), 1.68-1.62(\mathrm{~m}, 2 \mathrm{H}), 1.61-1.43(\mathrm{~m}, 8 \mathrm{H}), 1.38-1.27$ (m, 4H), 1.27-1.07 (m, 18H), $0.88(\mathrm{t}, J=7.4 \mathrm{~Hz}, 3 \mathrm{H}), 0.82-0.68(\mathrm{~m}, 6 \mathrm{H})$. The ${ }^{13} \mathrm{C}$ NMR spectrum of compound 21 is shown in Figure S23. ${ }^{13} \mathrm{C}$ NMR (DMSO- $d_{6}$, room temperature, $\left.100 \mathrm{MHz}\right) \delta(\mathrm{ppm}): 174.53,172.78$, $172.40,169.56,165.27,159.61,158.22,154.46,153.11,148.17,147.97,134.53,132.11$, $131.84,130.33,129.80,125.27,124.21,123.79,121.88,114.96,113.71,112.76,99.98,70.86$, $70.78,70.73,70.68,70.65,70.62,70.36,69.26,69.14,68.99,68.94,68.00,66.15,65.67,65.12$, $64.68,55.34,50.11,48.16,46.71,46.56,34.04,31.38,29.49,29.35,29.28,29.21,29.07,29.00$, $28.48,27.96,27.66,25.94,25.74,25.38,22.59,19.75,17.82,14.23,13.92,12.16$. HRESIMS is shown in Figure S24: $\mathrm{m} / z$ calcd for $\mathrm{C}_{68} \mathrm{H}_{101} \mathrm{~N}_{6} \mathrm{O}_{18}{ }^{+}, 1289.71669\left[\mathrm{M}-\mathrm{PF}_{6}\right]^{+}$; found $1289.72280\left[\mathrm{M}-\mathrm{PF}_{6}\right]^{+}$. 


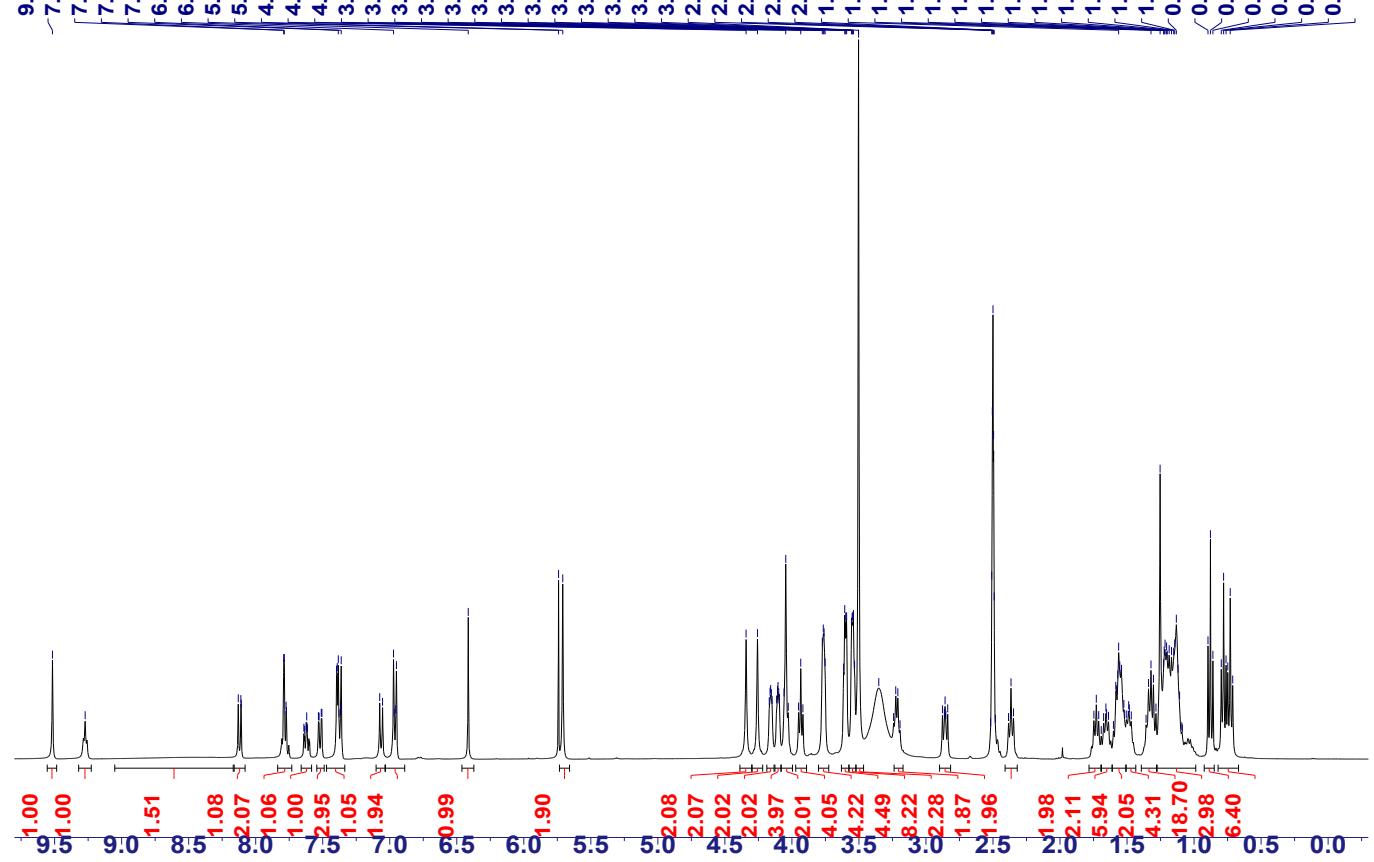

Figure S22. ${ }^{1} \mathrm{H}$ NMR spectrum (DMSO- $d_{6}$, room temperature, $400 \mathrm{MHz}$ ) of compound 21.

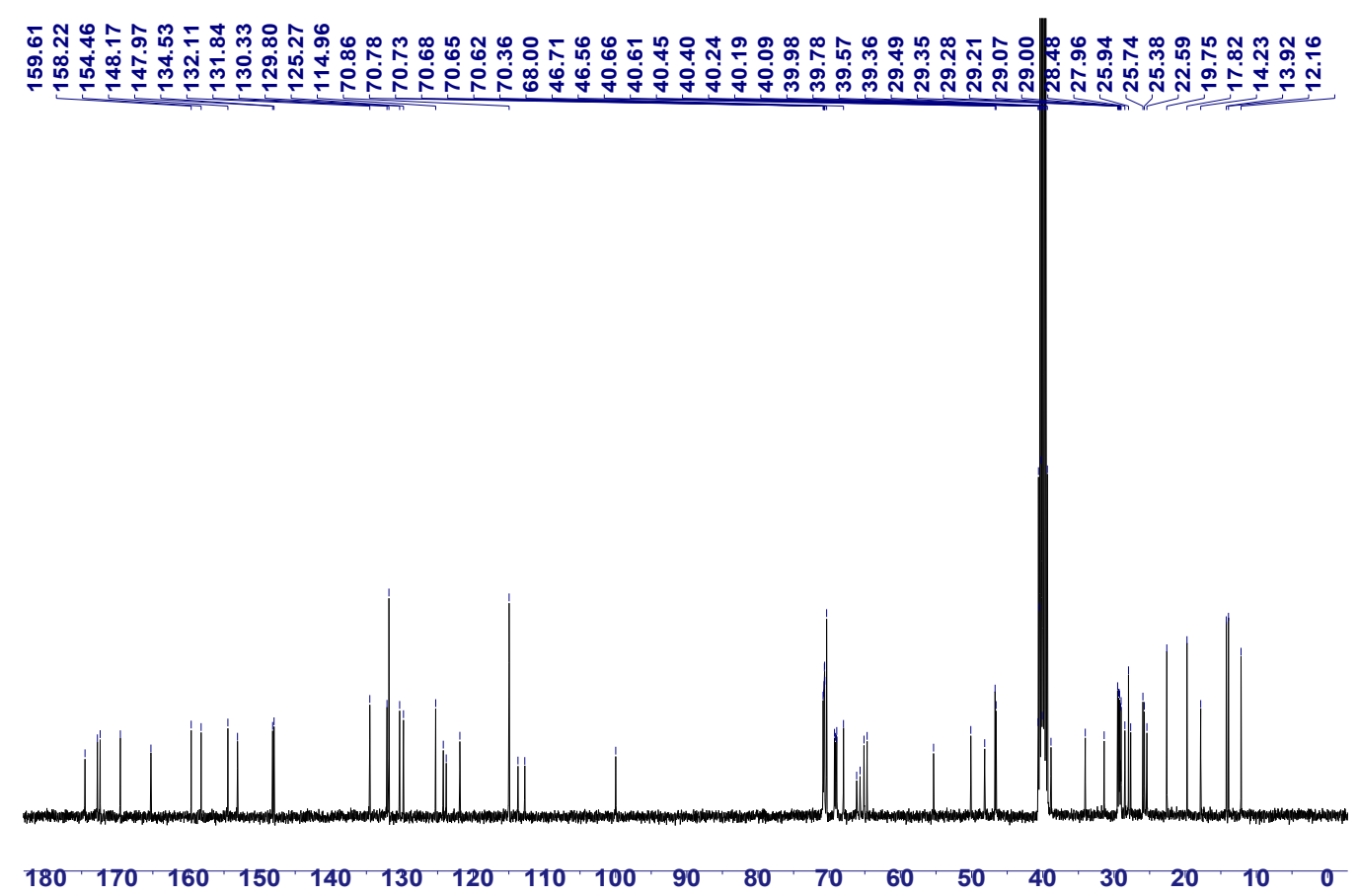

Figure S23. ${ }^{13} \mathrm{C}$ NMR spectrum (DMSO- $d_{6}$, room temperature, $100 \mathrm{MHz}$ ) of compound 21. 


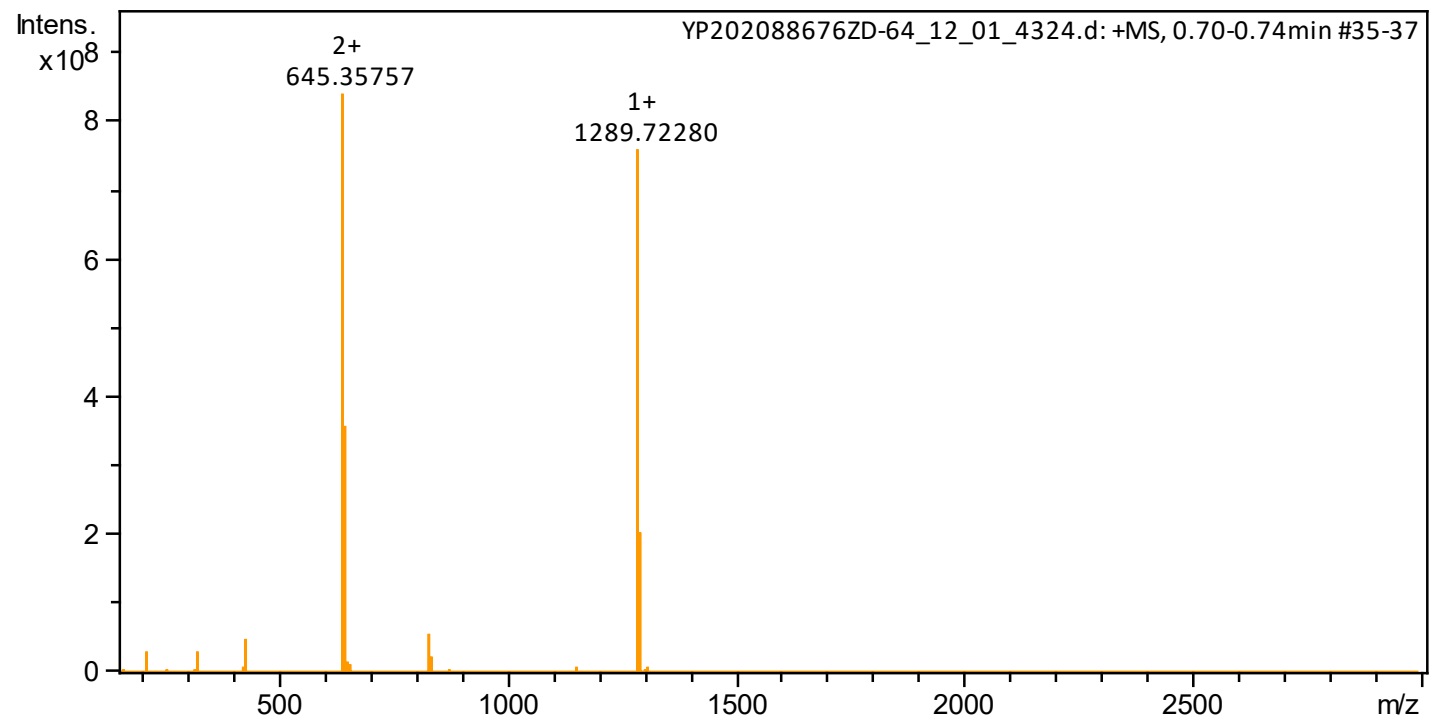

Figure S24. Electrospray ionization mass spectrum of compound 21. 


\section{Synthesis of CP-1}

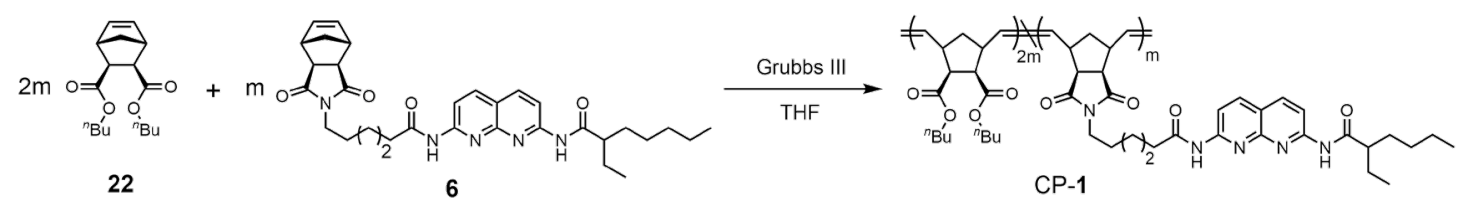

An oven-dried vial was charged with compound 22 ( $236 \mathrm{mg}, 0.801 \mathrm{mmol})$, compound 6 (218 $\mathrm{mg}, 0.399 \mathrm{mmol}$ ) and a stir bar. The vial was then degassed, and the $10 \mathrm{~mL}$ of degassed anhydrous THF was added via syringe under a nitrogen atmosphere to dissolve the monomer. The $\mathrm{Ru}$ catalyst ( $5.02 \mathrm{mg}, 5.68 \mu \mathrm{mol})$ in $2 \mathrm{~mL}$ degassed anhydrous THF was injected into the monomer solution to initiate the polymerization. The solution was stirred for $8 \mathrm{~h}$ at room temperature and then quenched by the addition of $0.1 \mathrm{~mL}$ of neat ethyl vinyl ether. After stirring for an additional $60 \mathrm{~min}$, the mixture was added dropwise to $200 \mathrm{~mL}$ of rapidly stirring methanol and a yellow-green precipitate formed immediately. The suspension was then centrifuged and collected. The solid was dried under vacuum for $24 \mathrm{~h}$ to afford CP-1 (368 mg, 81\%). The ${ }^{1} \mathrm{H}$ NMR spectrum of CP-1 is shown in Figure S25. The SEC curve of CP-1 is shown in Figure S26 with $M_{\mathrm{n}}=77000 \mathrm{~g} / \mathrm{mol}$ and $\oslash=1.96$. 


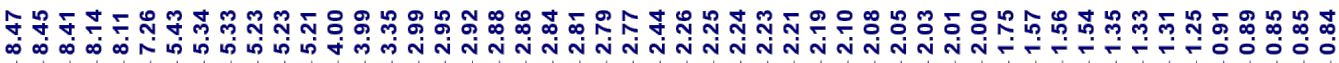
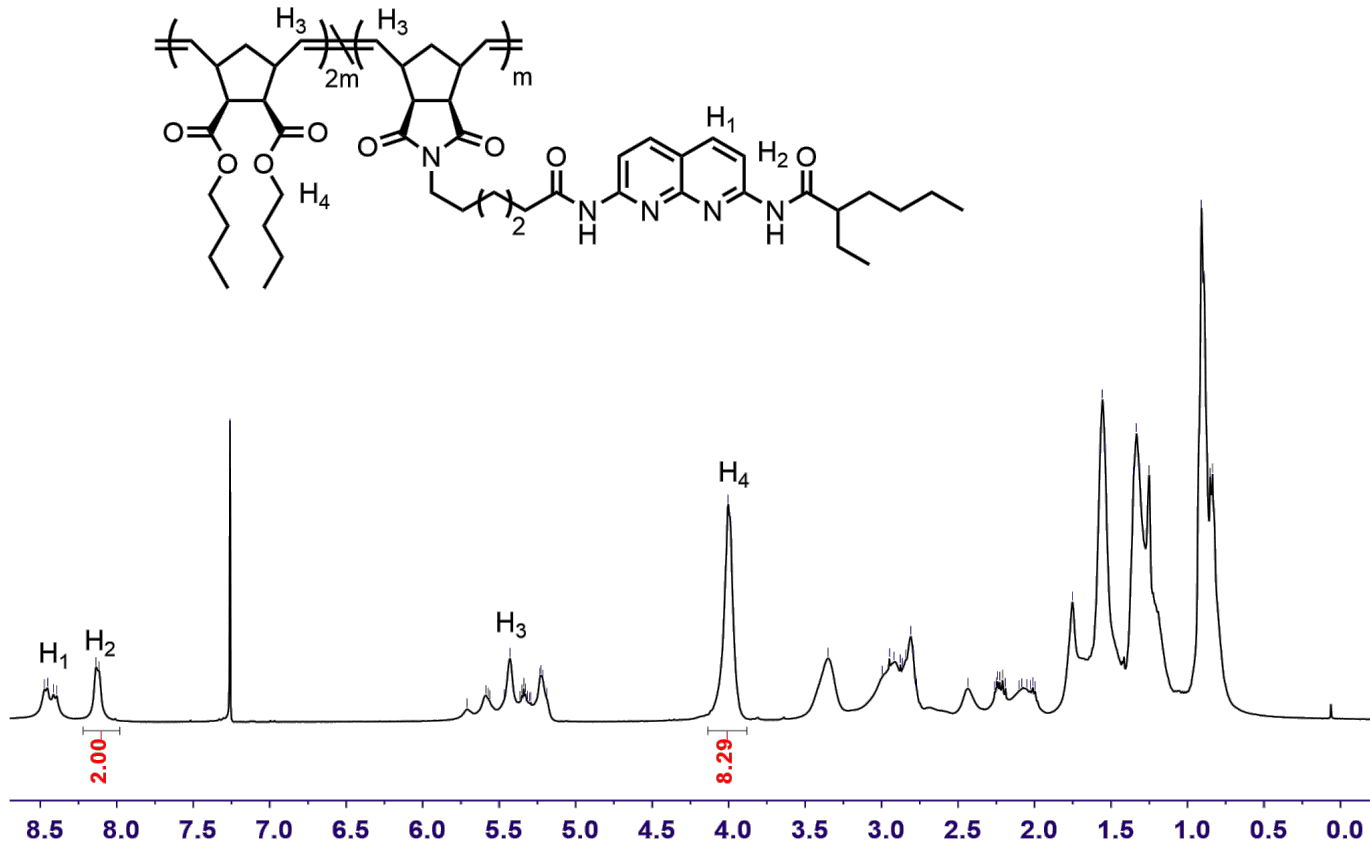

Figure S25. ${ }^{1} \mathrm{H}$ NMR spectrum $\left(\mathrm{CDCl}_{3}\right.$, room temperature, $\left.400 \mathrm{MHz}\right)$ of $\mathrm{CP}-1$.

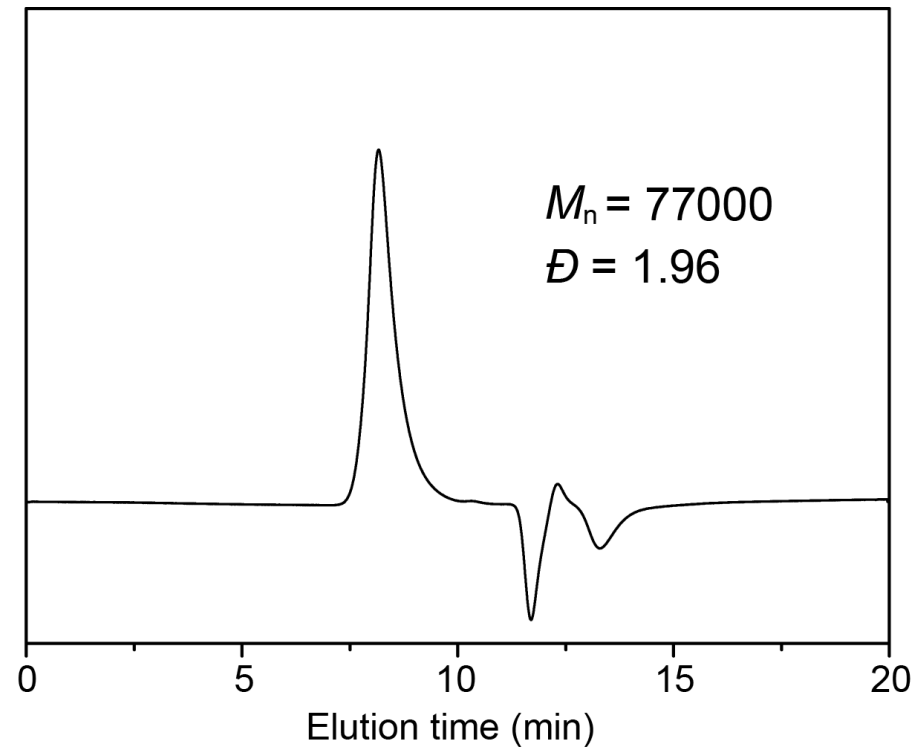

Figure S26. GPC elution curve of CP-1 with DMF as the eluent and PS as the standard. 
Synthesis of CP-2

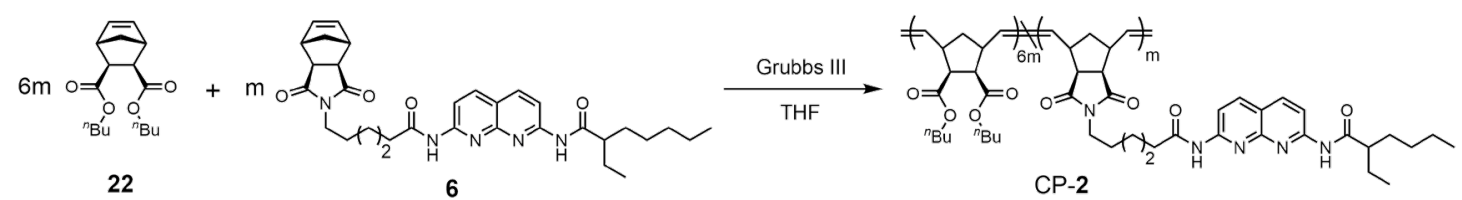

An oven-dried vial was charged with compound 22 (1.77g, $6.01 \mathrm{mmol})$, compound 6 (546 mg, $1.01 \mathrm{mmol}$ ) and a stir bar. The vial was then degassed, and the $26 \mathrm{~mL}$ of degassed anhydrous THF was added via syringe under a nitrogen atmosphere to dissolve the monomer. The $\mathrm{Ru}$ catalyst (25.6 mg, $28.9 \mu \mathrm{mol})$ in $2 \mathrm{~mL}$ degassed anhydrous THF was injected into the monomer solution to initiate the polymerization. The solution was stirred for $8 \mathrm{~h}$ at room temperature and then quenched by the addition of $0.2 \mathrm{~mL}$ of neat ethyl vinyl ether. After stirring for an additional $60 \mathrm{~min}$, the mixture was added dropwise to $500 \mathrm{~mL}$ of rapidly stirring methanol and a yellowgreen precipitate formed immediately. The suspension was then centrifuged and collected. The solid was dried under vacuum for $24 \mathrm{~h}$ to afford CP-2 (1.99 g, 86\%). The ${ }^{1} \mathrm{H}$ NMR spectrum of CP-2 is shown in Figure S27. The SEC curve of CP-2 is shown in Figure S28 with $M_{\mathrm{n}}=73000$ $\mathrm{g} / \mathrm{mol}$ and $Ð=1.79$. 


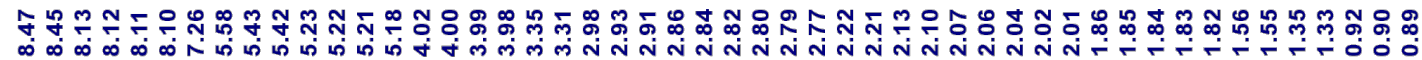<smiles>C=CC1CC(CC(C)(C)C)C(C(=O)OCCCCC)C1C(=O)OCCCCC</smiles>

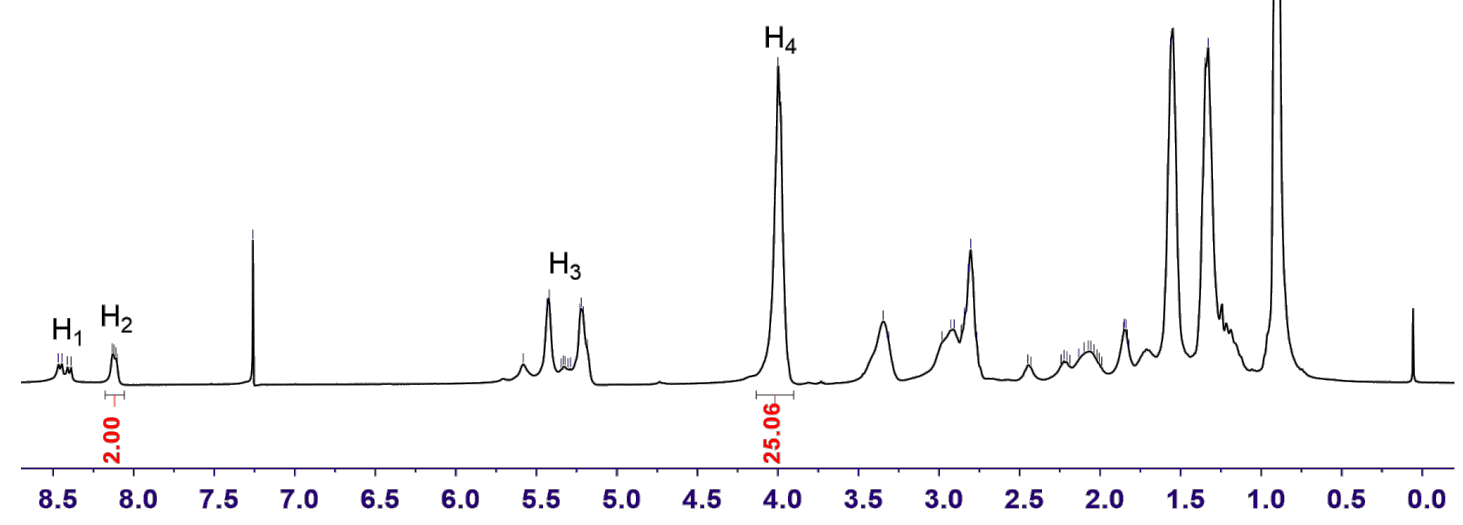

Figure S27. ${ }^{1} \mathrm{H}$ NMR spectrum $\left(\mathrm{CDCl}_{3}\right.$, room temperature, $\left.400 \mathrm{MHz}\right)$ of $\mathrm{CP}-2$.

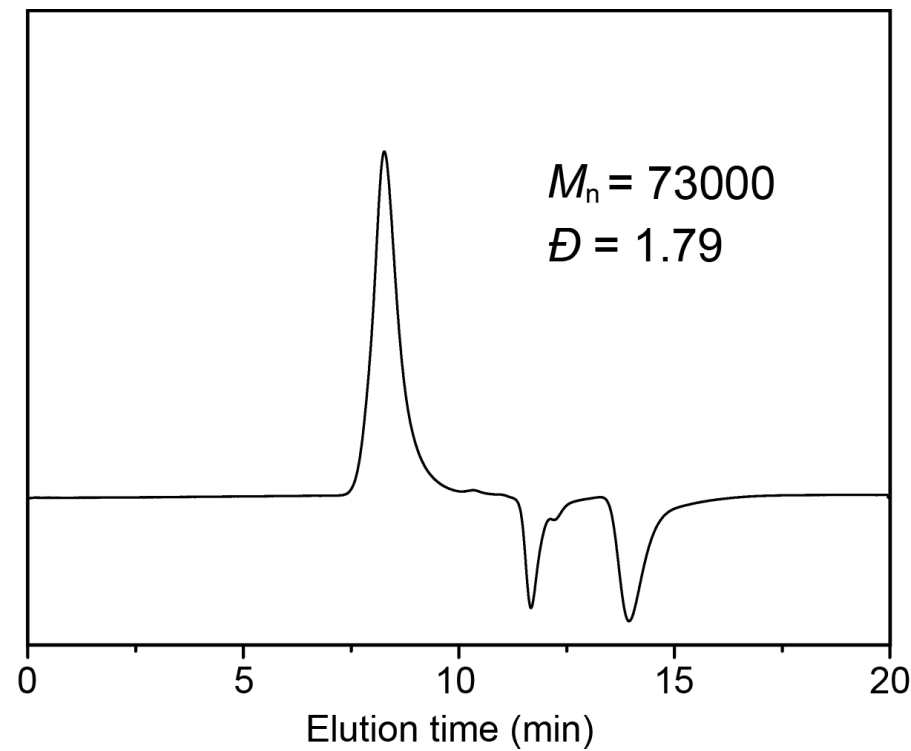

Figure S28. GPC elution curve of CP-2 with DMF as the eluent and PS as the standard. 
Synthesis of CP-3

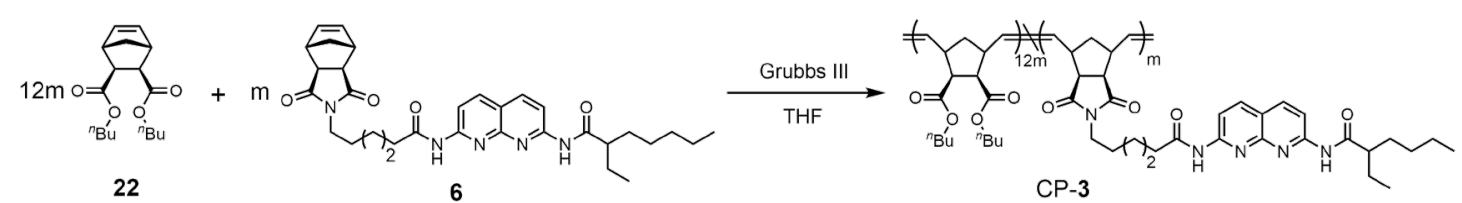

An oven-dried vial was charged with compound 22 (1.41g, $4.79 \mathrm{mmol})$, compound 6 (218 mg, $0.399 \mathrm{mmol}$ ) and a stir bar. The vial was then degassed, and the $26 \mathrm{~mL}$ of degassed anhydrous THF was added via syringe under a nitrogen atmosphere to dissolve the monomer. The $\mathrm{Ru}$ catalyst (25.6 mg, $20.4 \mu \mathrm{mol})$ in $2 \mathrm{~mL}$ degassed anhydrous THF was injected into the monomer solution to initiate the polymerization. The solution was stirred for $8 \mathrm{~h}$ at room temperature and then quenched by the addition of $0.2 \mathrm{~mL}$ of neat ethyl vinyl ether. After stirring for an additional $60 \mathrm{~min}$, the mixture was added dropwise to $500 \mathrm{~mL}$ of rapidly stirring methanol and a yellowgreen precipitate formed immediately. The suspension was then centrifuged and collected. The solid was dried under vacuum for $24 \mathrm{~h}$ to afford CP-3 (1.76 g, 79\%). The ${ }^{1} \mathrm{H}$ NMR spectrum of CP-3 is shown in Figure S29. The SEC curve of CP-3 is shown in Figure S30 with $M_{\mathrm{n}}=81000$ $\mathrm{g} / \mathrm{mol}$ and $Ð=1.73$. 

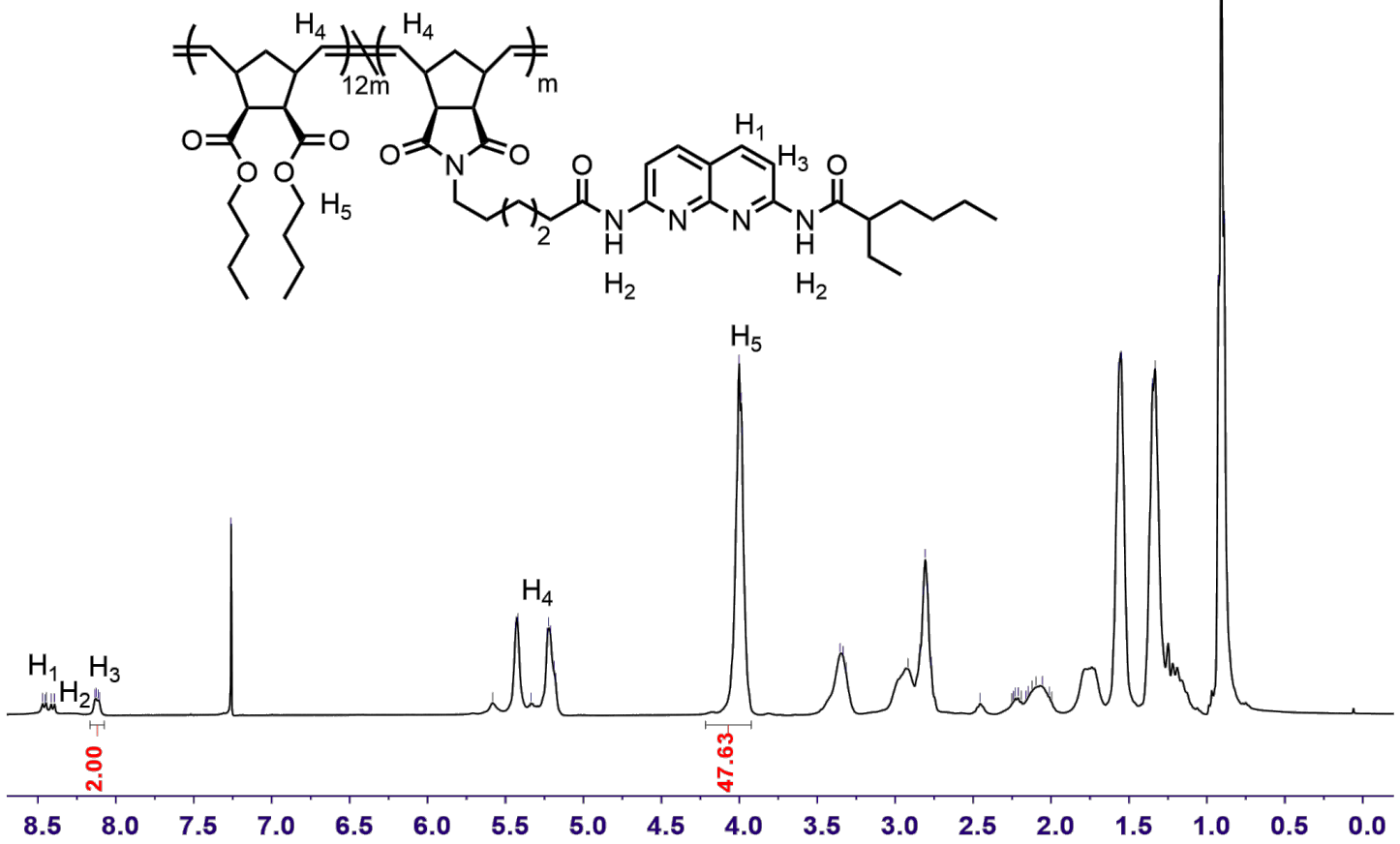

Figure S29. ${ }^{1} \mathrm{H}$ NMR spectrum $\left(\mathrm{CDCl}_{3}\right.$, room temperature, $\left.400 \mathrm{MHz}\right)$ of $\mathrm{CP}-3$.

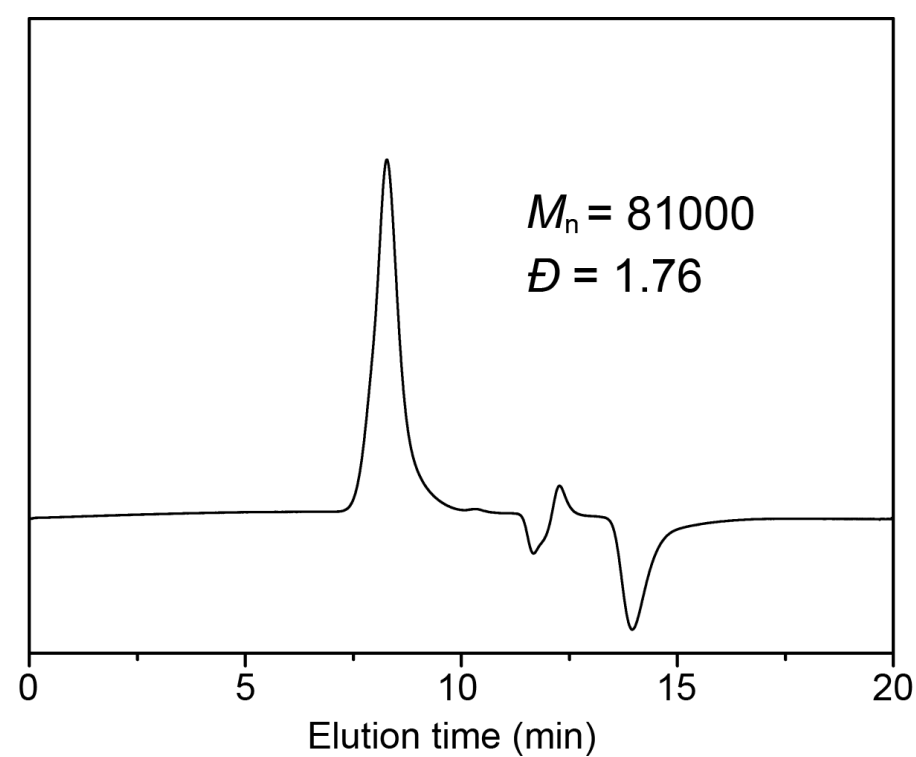

Figure S30. GPC elution curve of CP-3 with DMF as the eluent and PS as the standard. 


\section{Syntheses of CSPs-1-3}

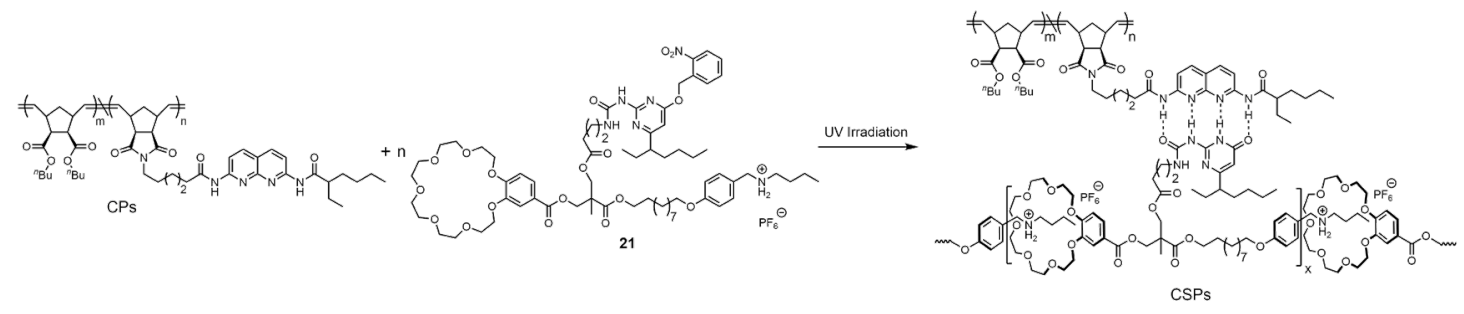

A vial was charged with CPs and compound 21 in a 1:1 molar ratio of DAN and UPy moieties. Then stir bar and DCM were added. The solution was stirred and irradiated by UV-LED with the wavelength of $365 \mathrm{~nm}$. After $1.5 \mathrm{~h}$ irradiation, the solution was concentrated and poured into a teflon mould to allow the solvent to evaporate slowly. The solid samples were further dried under vacuum for $24 \mathrm{~h}$. The ${ }^{1} \mathrm{H}$ NMR spectra of CSPs-1-3 are shown in Figures S29-S31, respectively.

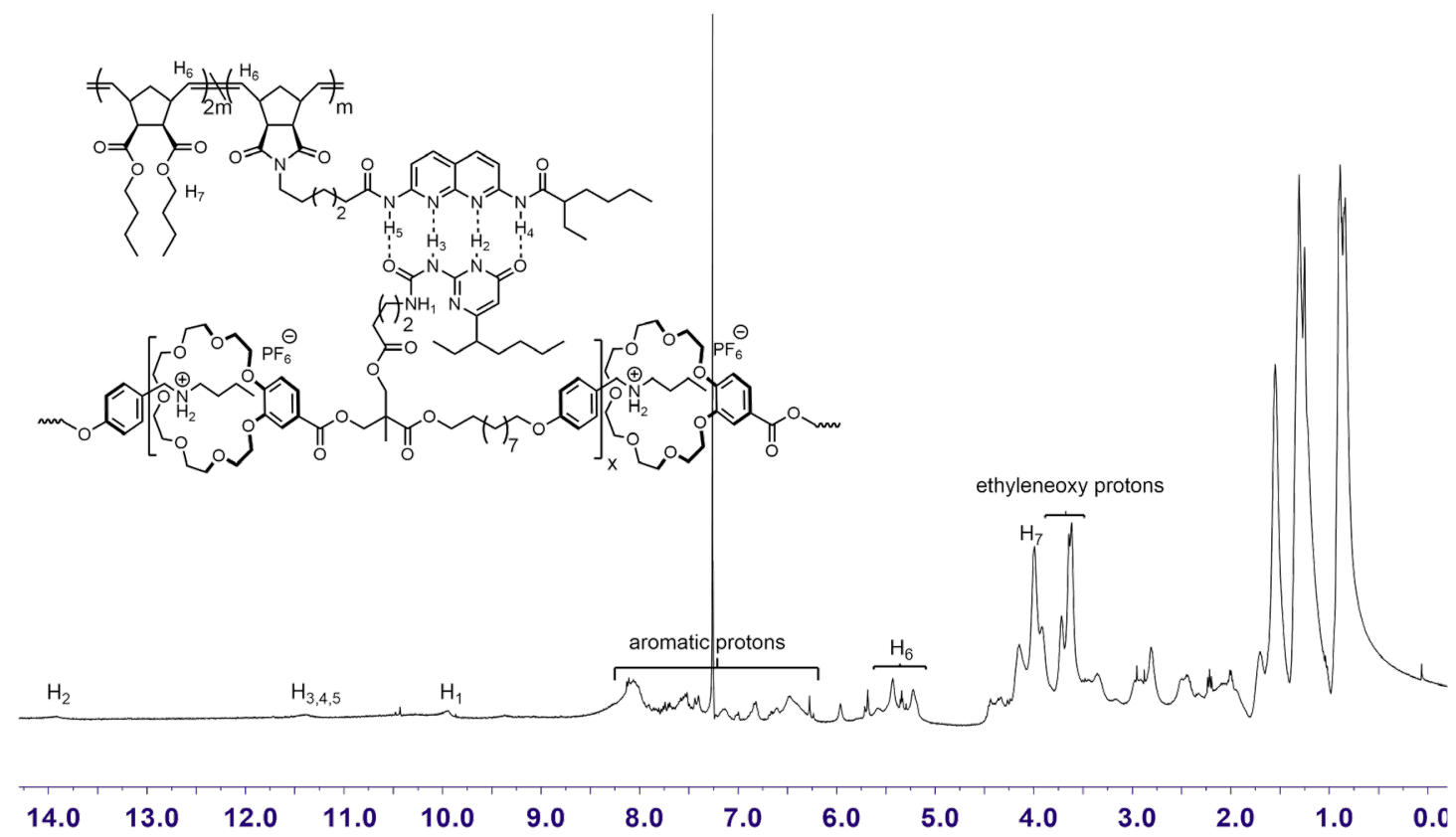

Figure S31. ${ }^{1} \mathrm{H}$ NMR spectrum $\left(\mathrm{CDCl}_{3}\right.$, room temperature, $\left.400 \mathrm{MHz}\right)$ of CSP-1. 


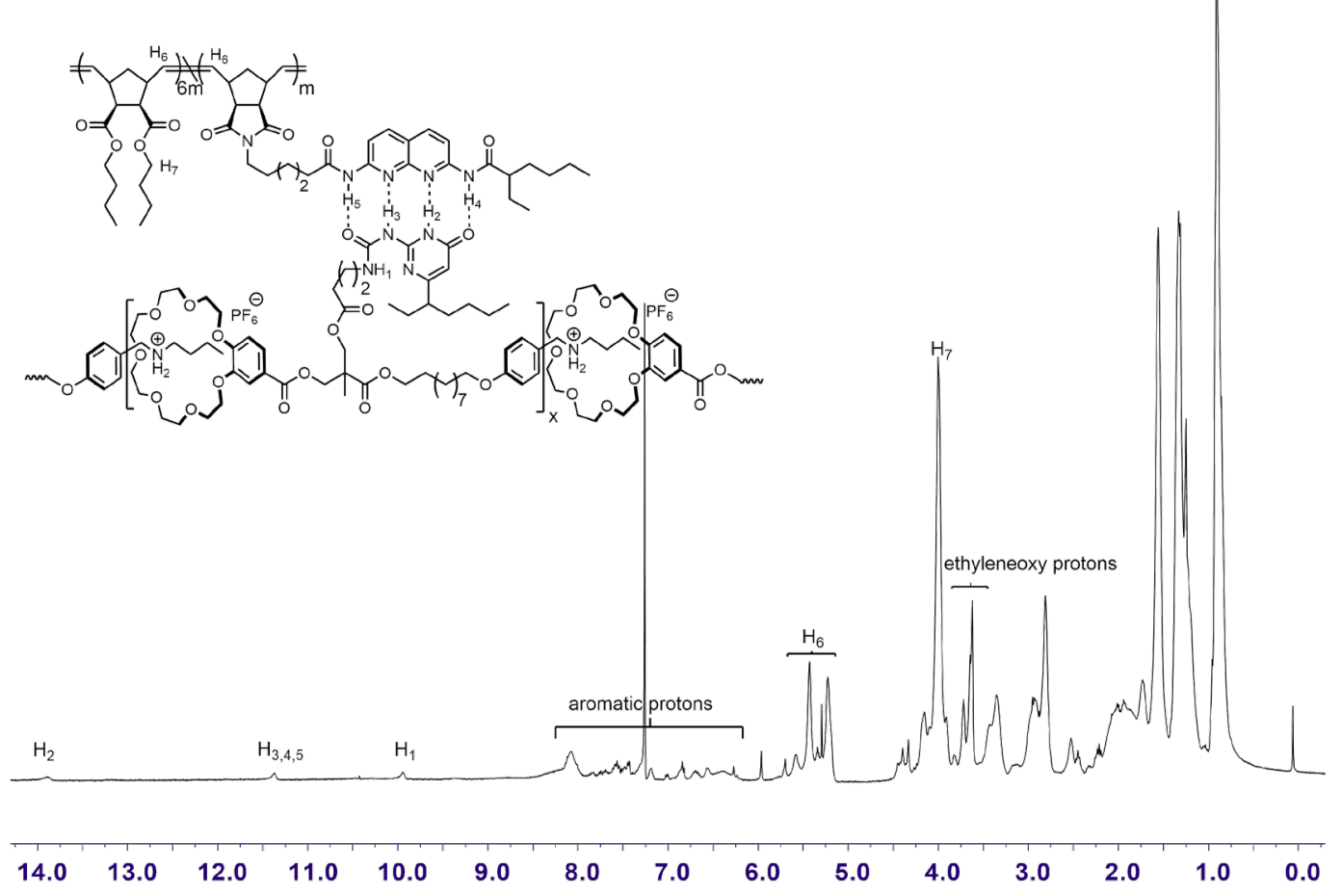

Figure S32. ${ }^{1} \mathrm{H}$ NMR spectrum $\left(\mathrm{CDCl}_{3}\right.$, room temperature, $\left.400 \mathrm{MHz}\right)$ of CSP-2.
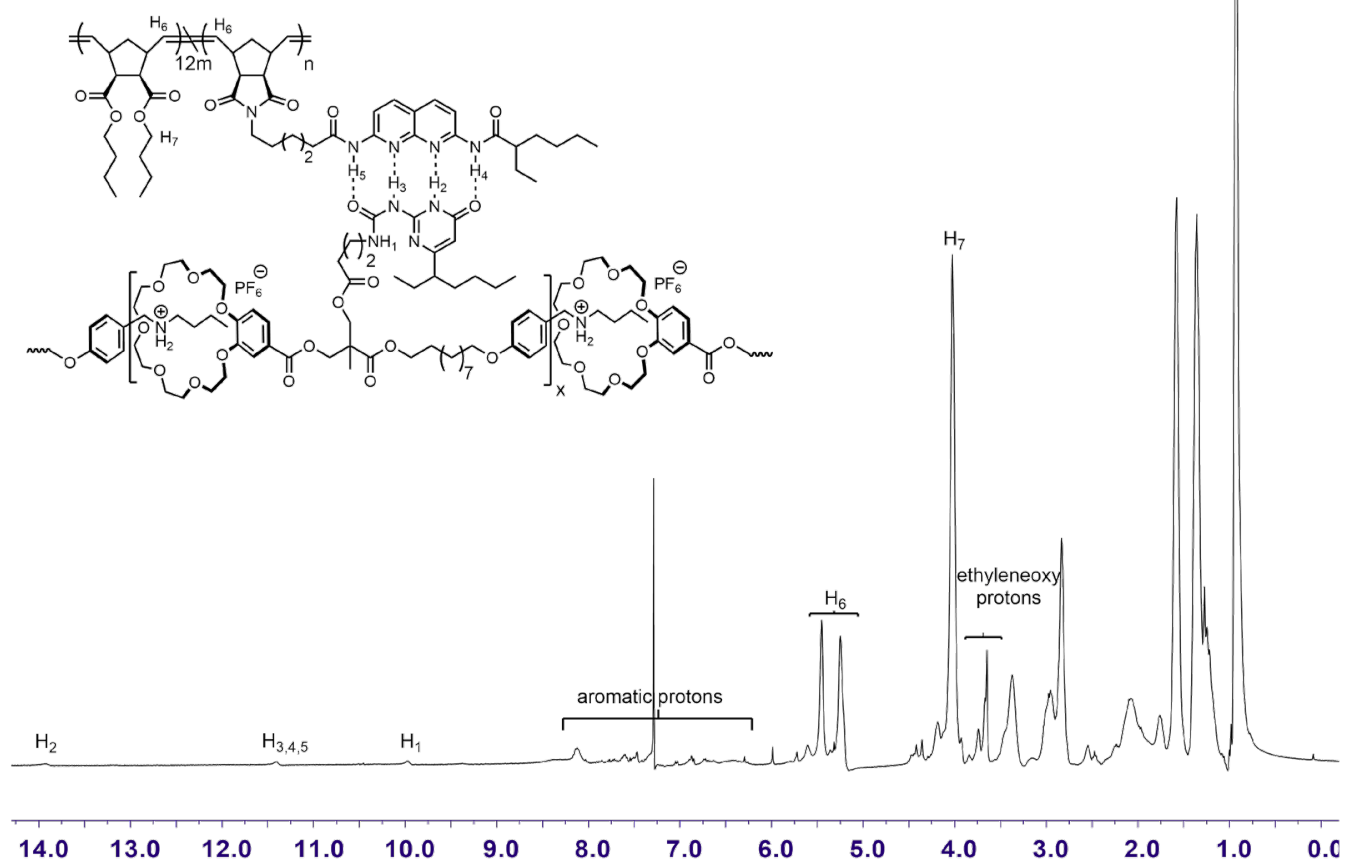

Figure S33. ${ }^{1} \mathrm{H}$ NMR spectrum $\left(\mathrm{CDCl}_{3}\right.$, room temperature, $\left.400 \mathrm{MHz}\right)$ of CSP-3. 


\section{Synthesis of control-1}

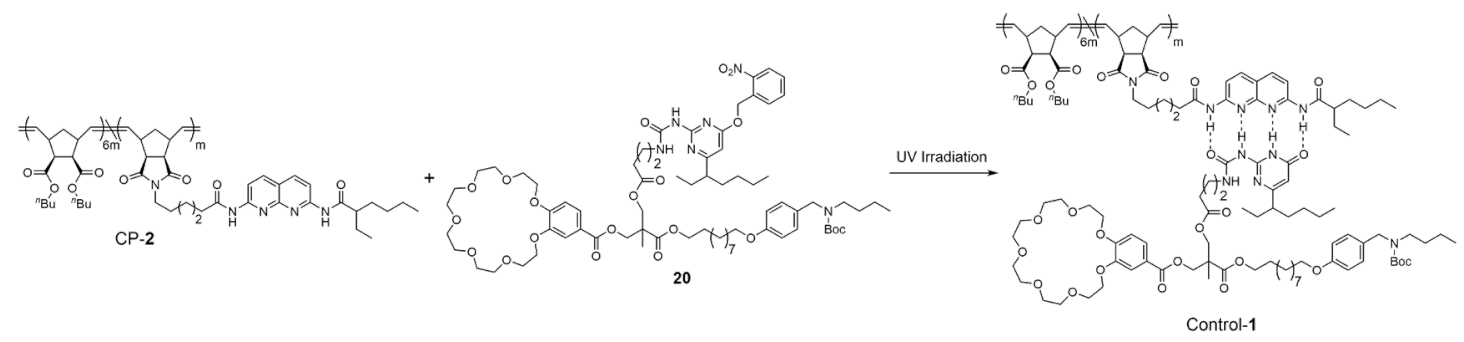

CP-2 (277 mg, $1.20 \mathrm{mmol}$ (DAN moiety)), and compound 20 (167 mg, $1.20 \mathrm{mmol}$ ) were dissolved in $6 \mathrm{~mL}$ DCM. The solution was stirred and irradiated by LED lamp with the wavelength of $365 \mathrm{~nm}$. After $1.5 \mathrm{~h}$ irradiation, the solution was concentrated and poured into a teflon mould to allow the solvent to evaporate slowly. The solid samples were further dried under vacuum for $24 \mathrm{~h}$. The ${ }^{1} \mathrm{H}$ NMR spectrum of control-1 was shown in Figure S32.
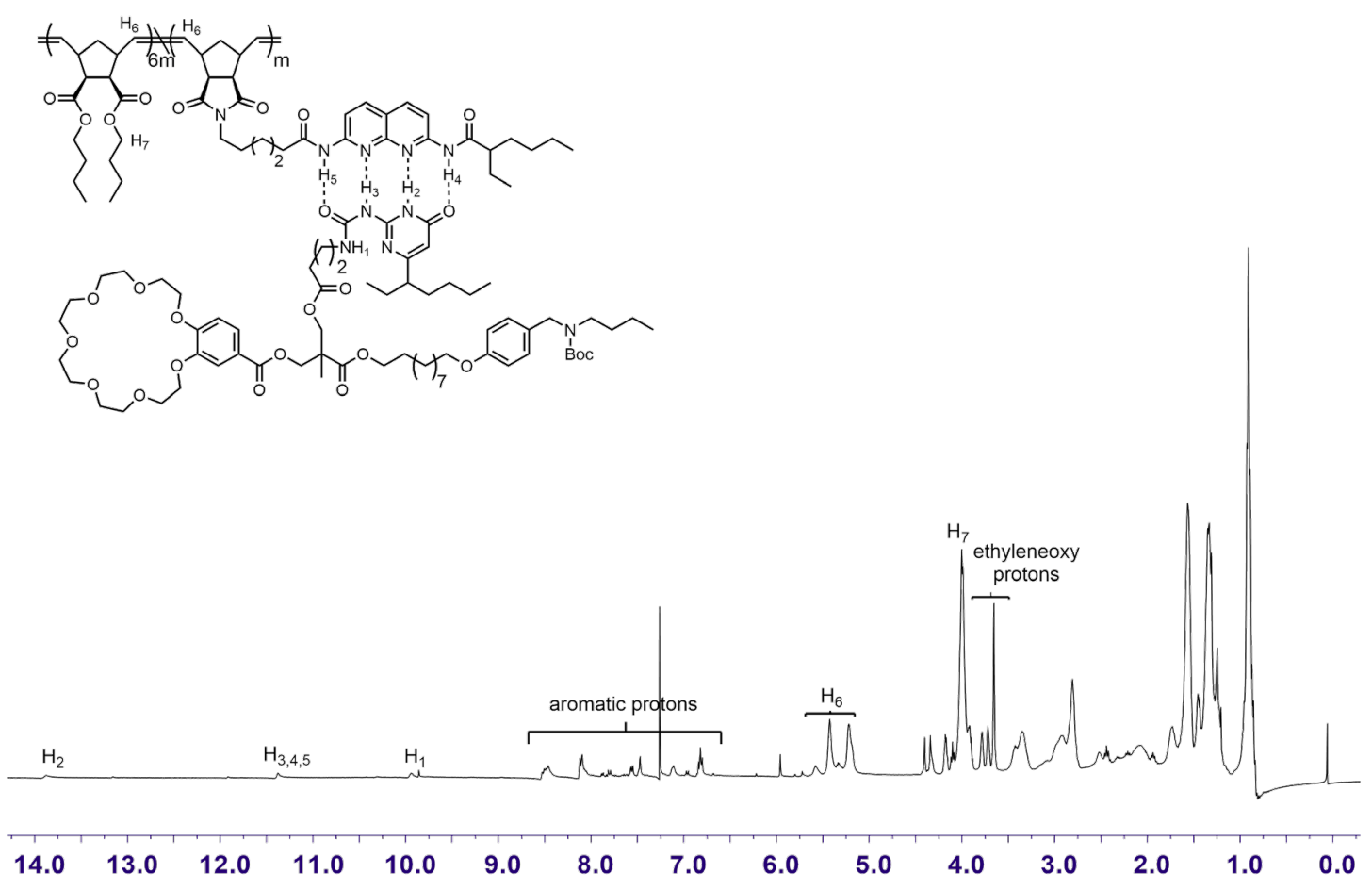

Figure S34. ${ }^{1} \mathrm{H}$ NMR spectrum $\left(\mathrm{CDCl}_{3}\right.$, room temperature, $\left.400 \mathrm{MHz}\right)$ of control-1. 


\section{Synthesis of control-2}

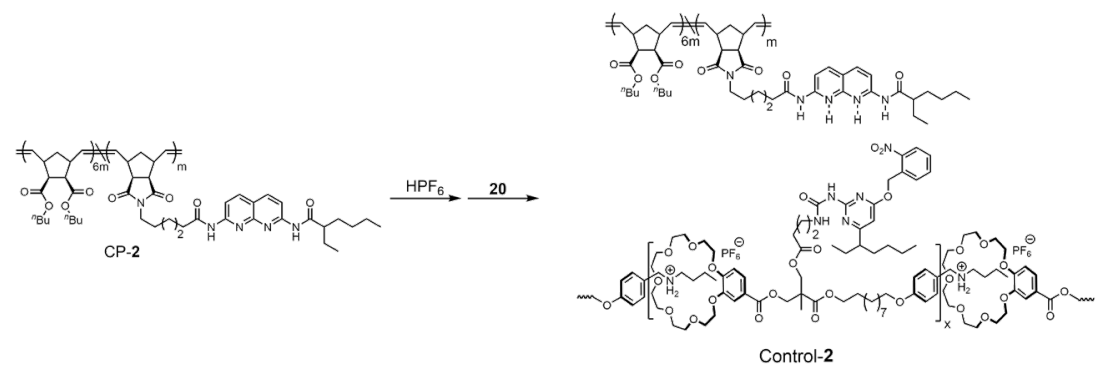

CP-2 (277 mg, $1.20 \mathrm{mmol}$ (DAN moiety)) was dissolved in $5 \mathrm{~mL}$ acetone, and then excess $\mathrm{HPF}_{6}$ solution was added. The mixture was stirred at room temperature for $1 \mathrm{~h}$, and then was added dropwise to rapidly stirring water. The product was collected and dissolved in $5 \mathrm{~mL}$ acetone again. Compound $\mathbf{2 0}(166 \mathrm{mg}, 1.19 \mathrm{mmol})$ was added to the solution. After stirring for $2 \mathrm{~h}$, the solution was concentrated and poured into a teflon mould to allow the solvent to evaporate slowly. The solid samples were further dried under vacuum for $24 \mathrm{~h}$. The ${ }^{1} \mathrm{H}$ NMR spectrum of control-2 was shown in Figure S33.

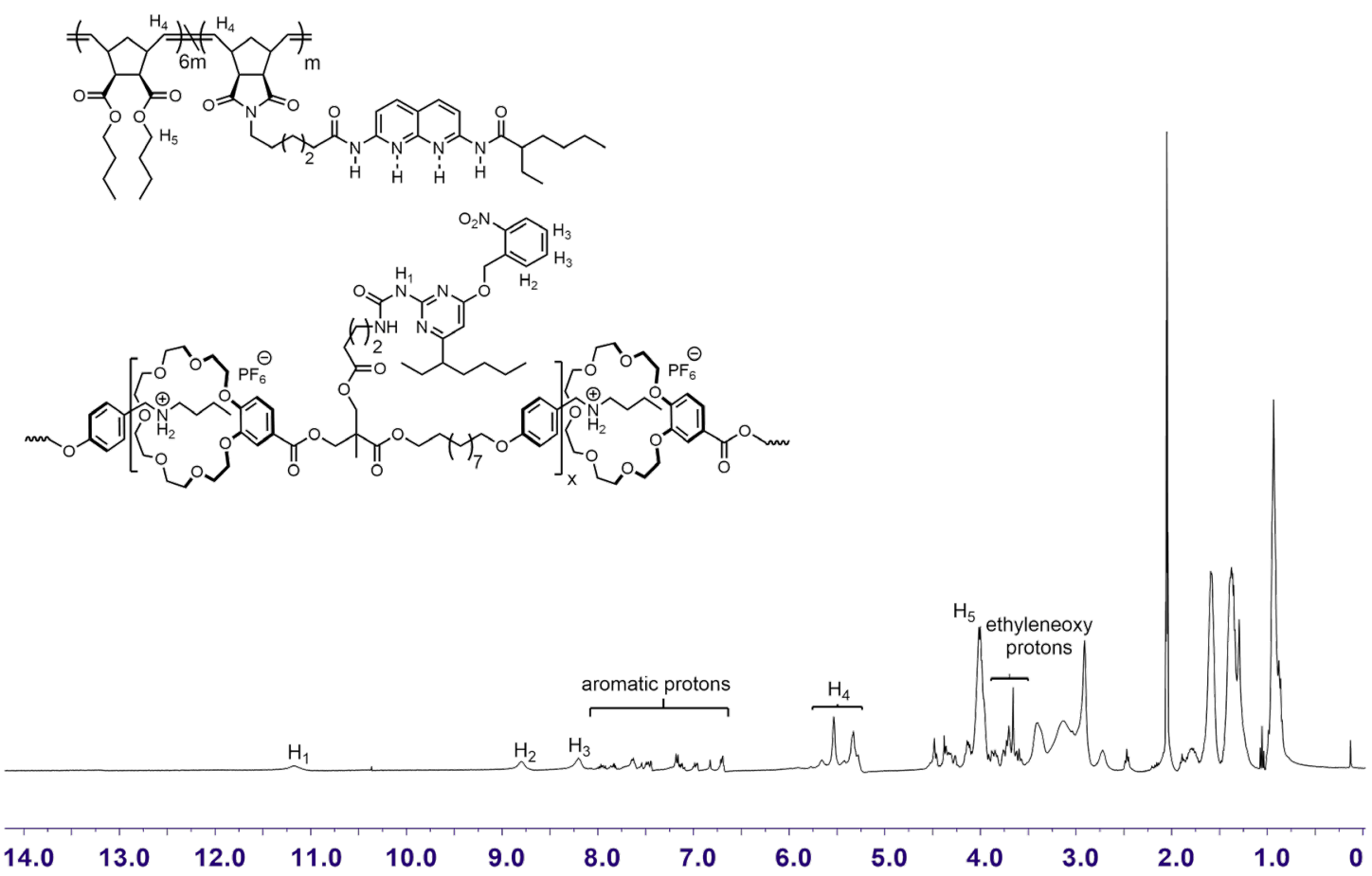

Figure S35. ${ }^{1} \mathrm{H}$ NMR spectrum (Acetone- $d_{6}$, room temperature, $400 \mathrm{MHz}$ ) of control-2. 

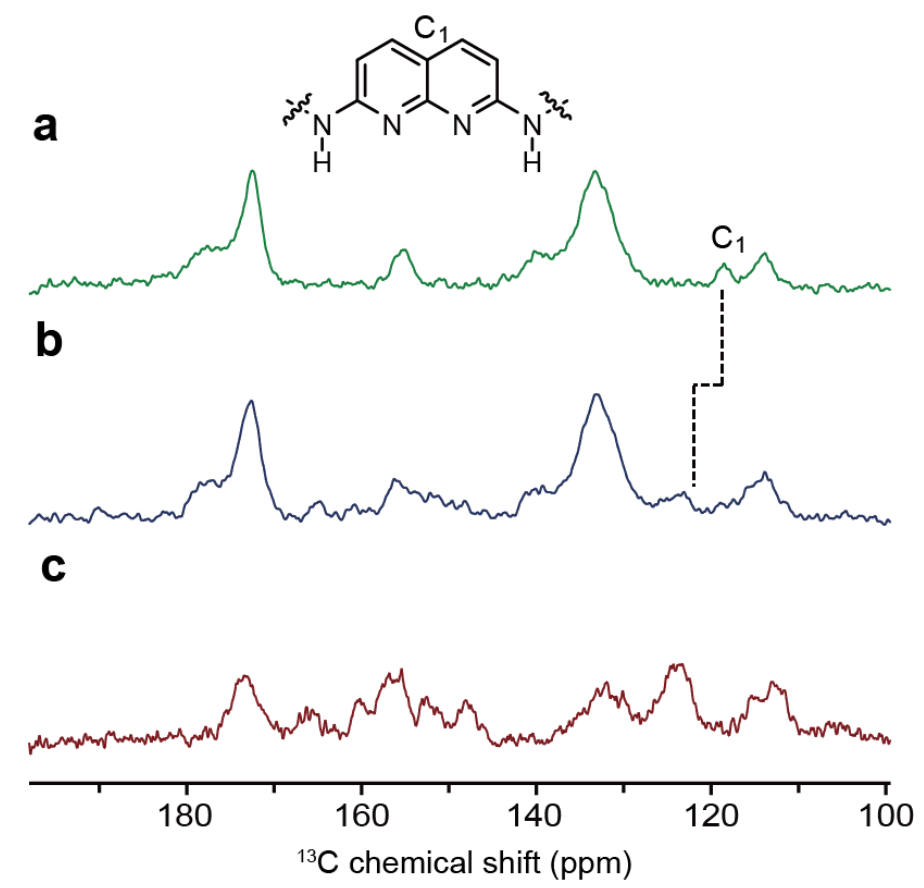

Figure S36. Partial ${ }^{13} \mathrm{C}$ CP/MAS solid-state NMR (150 MHz, $298 \mathrm{~K}$ ) of the CP-2 (a), CSP-2 (b) and pure SP after irradiation (c). In the spectrum of CSP-2, the signal of $\mathrm{C}_{1}$ on DAN moiety almost disappeared, and shifted downfield, indicating the formation of heterocomplementary quadruple H-bonding.

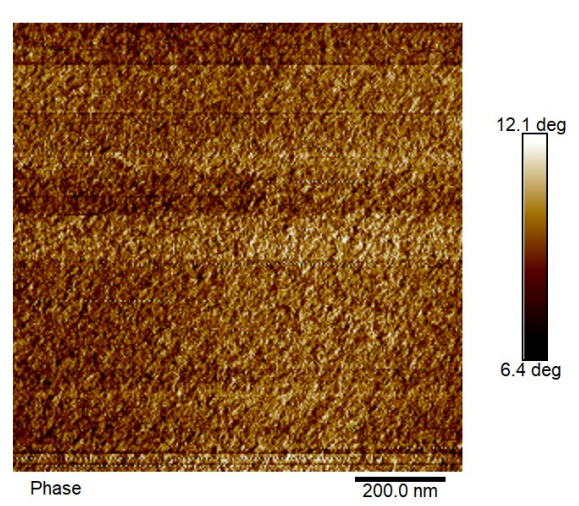

Figure S37. AFM phase image of control-1. Different from the results of control-2, no obvious phase separation could be observed, which further confirms the speculation that the formation of heterocomplementary quadruple H-bonding is beneficial to the blend of the two components to inhibit their self-aggregation. 

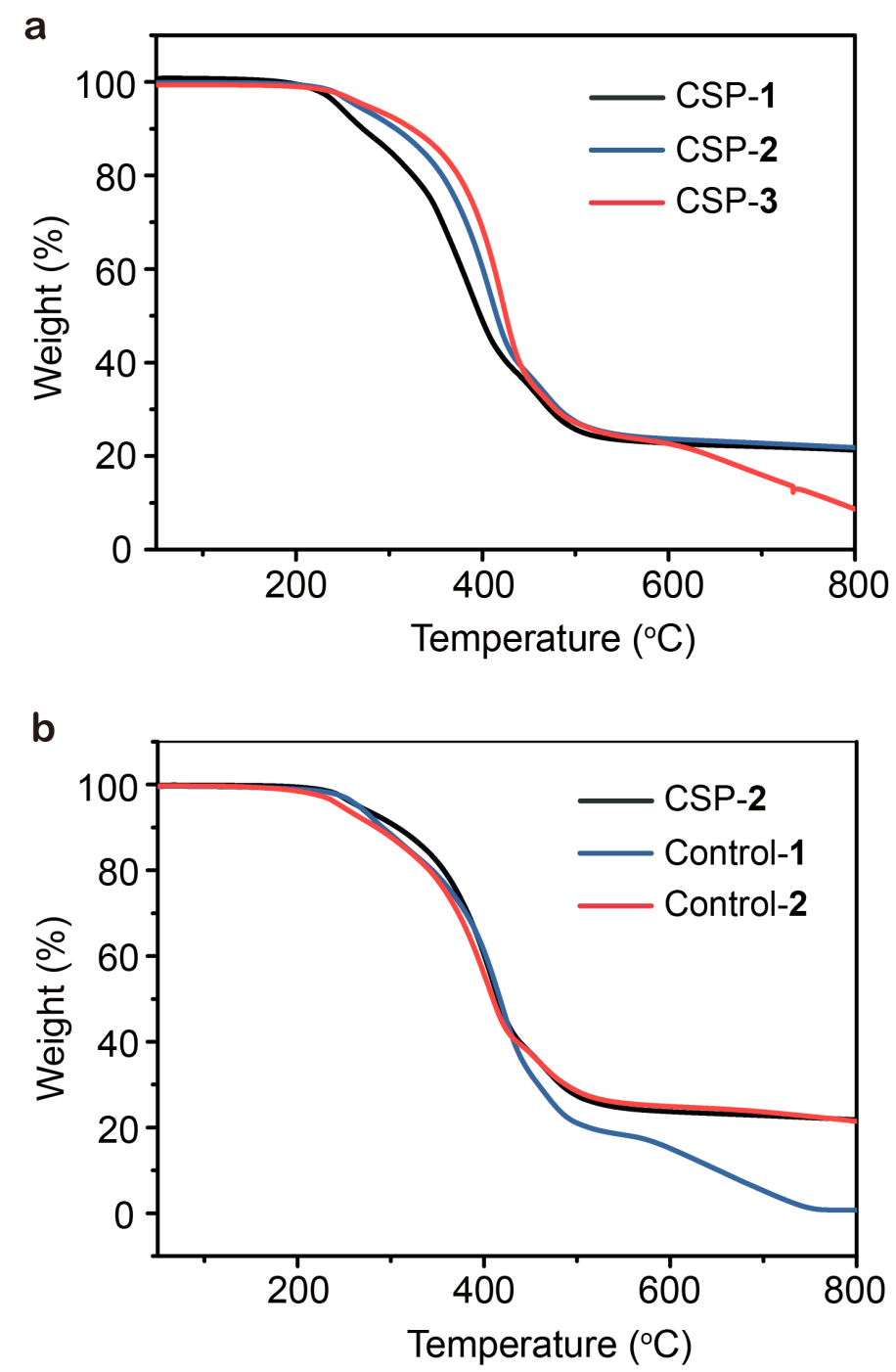

Figure S38. TGA curves of CSPs-1-3 (a) and CSP-2 as well as controls-1 and 2 (b) recorded under $\mathrm{N}_{2}$ flow $(50 \mathrm{~mL} / \mathrm{min})$ with a heating rate of $20^{\circ} \mathrm{C} / \mathrm{min}$. 

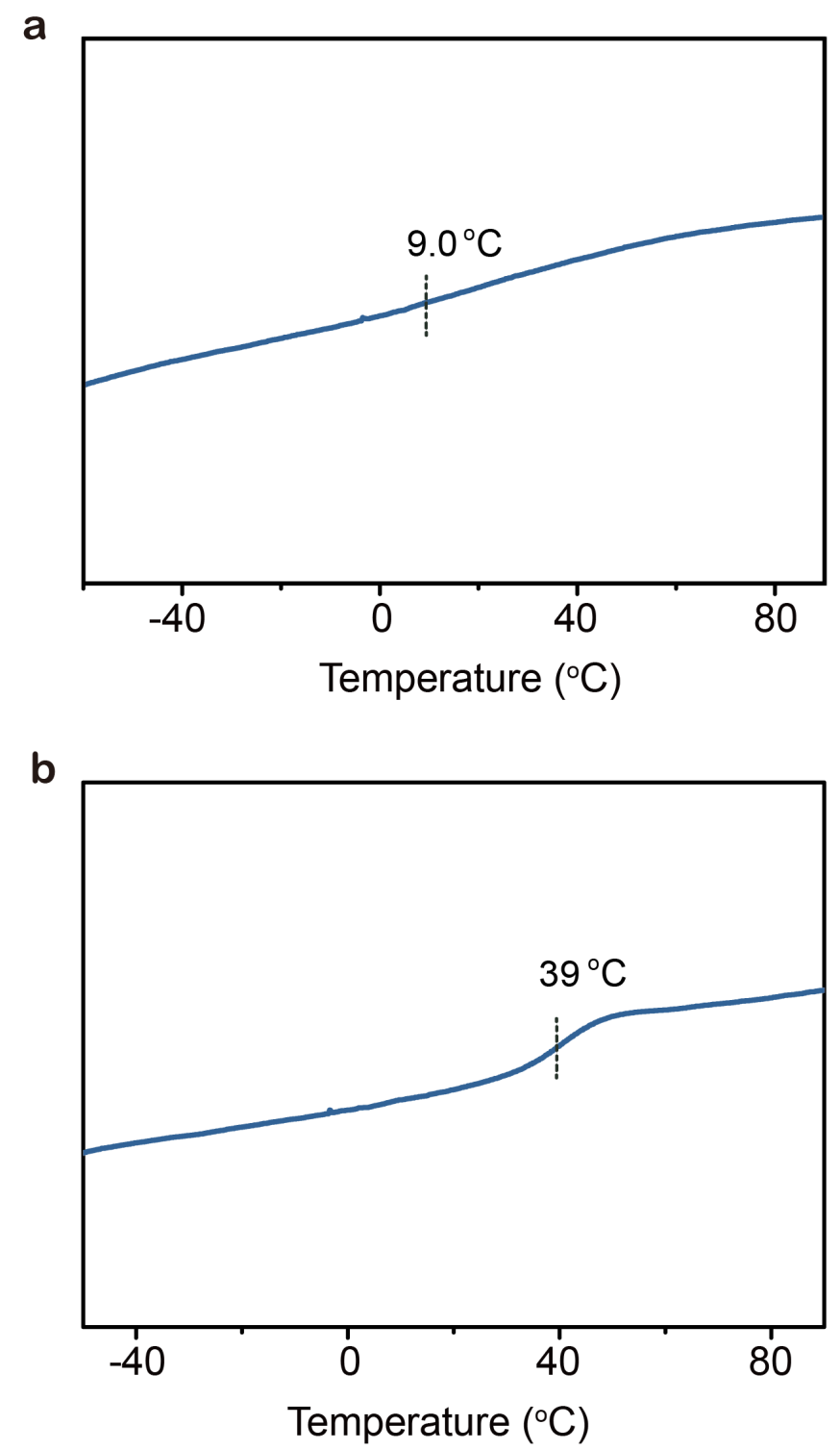

Figure S39. DSC curves of CP-2 (a) and pure SP after irradiation (b) recorded by the second heating scan from -50 to $90{ }^{\circ} \mathrm{C}$ with a heating rate of $20{ }^{\circ} \mathrm{C} / \mathrm{min}$. 


\begin{tabular}{ccccc}
\hline Ref. & Stress (MPa) & Strain (\%) & $\begin{array}{c}\text { Young's modulus } \\
\text { (MPa) }\end{array}$ & $\begin{array}{c}\text { Toughness } \\
\left(\mathbf{M J} / \mathbf{m}^{3}\right)\end{array}$ \\
\hline CSP-2 & 12.0 & 1506 & 145 & 136 \\
S7 & 132 & 40.0 & 3500 & 43.0 \\
S8 & 1.40 & 600 & 21.1 & 7.80 \\
S9 & 2.80 & 275 & - & 5.40 \\
S10 & 4.13 & 808 & 39.2 & 23.0 \\
S11 & 4.60 & 520 & 22.6 & 22.0 \\
S12 & 11.4 & 25.0 & - & 1.70 \\
S13 & 30.7 & 623 & 2.40 & 10.4 \\
S14 & 3.60 & 640 & - & 9.20 \\
S15 & 5.00 & 90.0 & - & 4.00 \\
S16 & 1.92 & 780 & 17.3 & 10.0 \\
S17 & 3.20 & 500 & 0.30 & 1.70 \\
\hline
\end{tabular}

Table S1. Comparison of the mechanical properties of CSP-2 with the reported supramolecular polymer networks in the bulk state. 

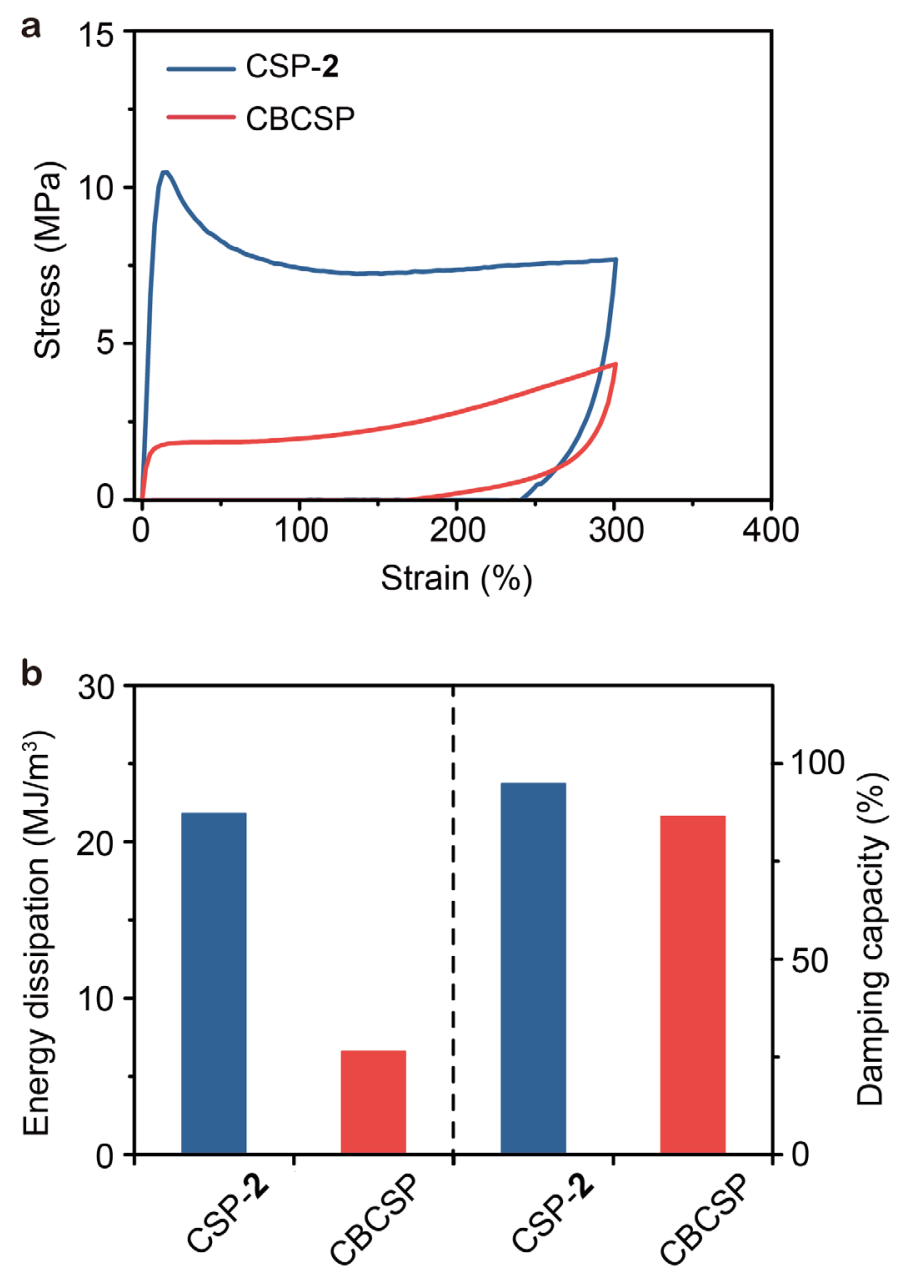

Figure S40. (a) Cyclic tensile test curves of CSP-2 and covalent bond connected CSP (CBCSP) at a strain of $300 \%$ under the deformation rate of $100 \mathrm{~mm} / \mathrm{min}$. (b) Energy dissipation and damping capacity of CSP-2 and CBCSP calculated based on the loading/unloading curves. 


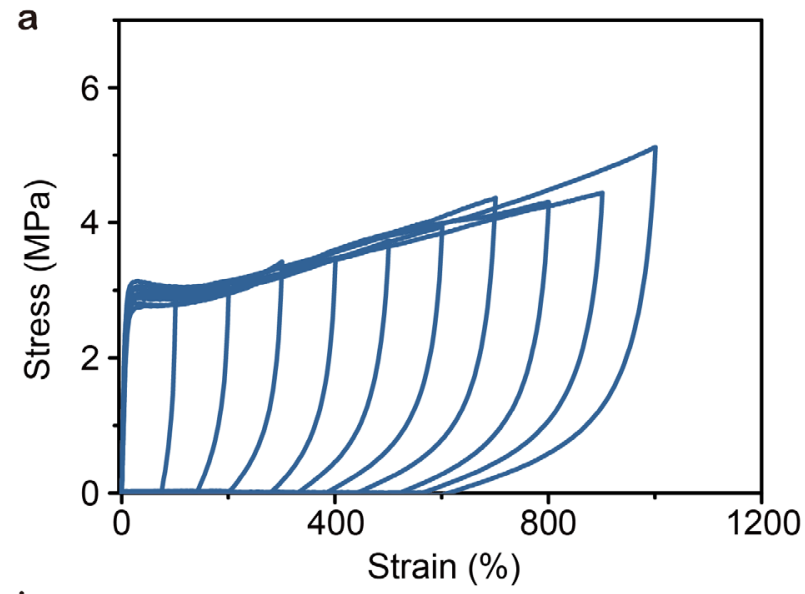

b

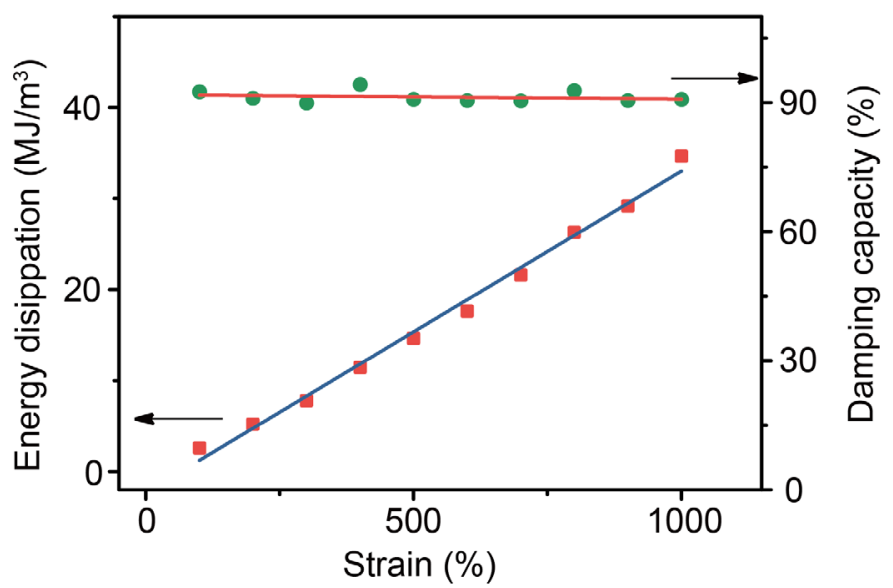

Figure S41. (a) Cyclic tensile test curves of CSP-3 recorded with increased maximum strains.

(b) Energy dissipation and damping capacity for each circle of the cyclic tensile test curves. 


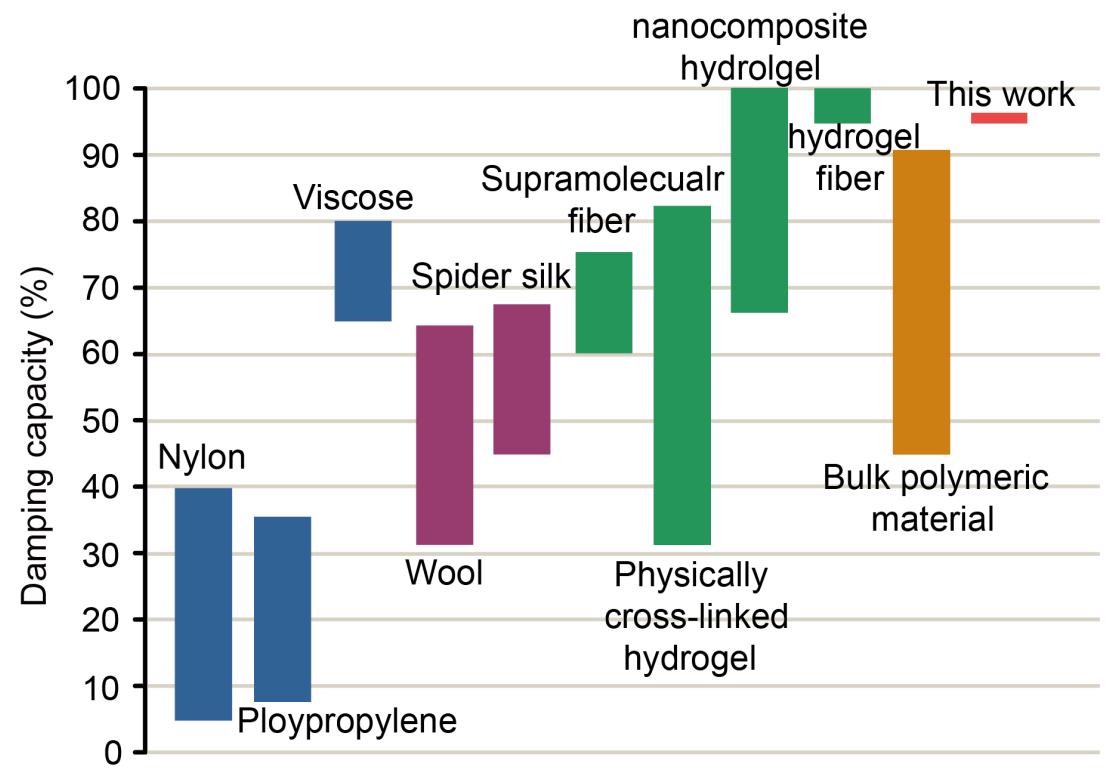

Figure S42. Comparison of the damping capacity of CSP-2 with other typical energydissipation materials. Corresponding data are extracted from the references S19-S32. 

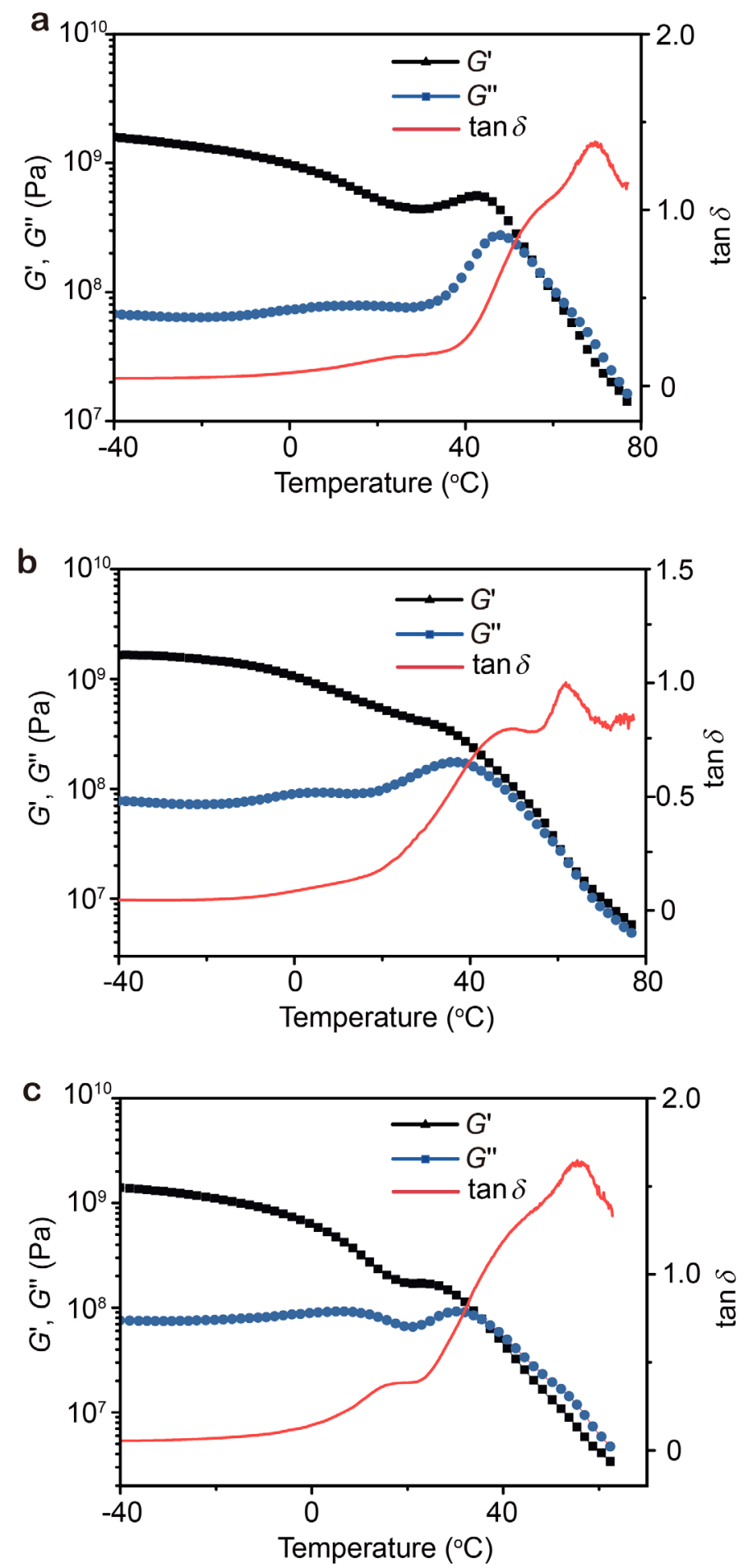

Figure S43. DMA temperature sweeps of CSP-2 (a), CSP-3 (b), and control-2 (c). 


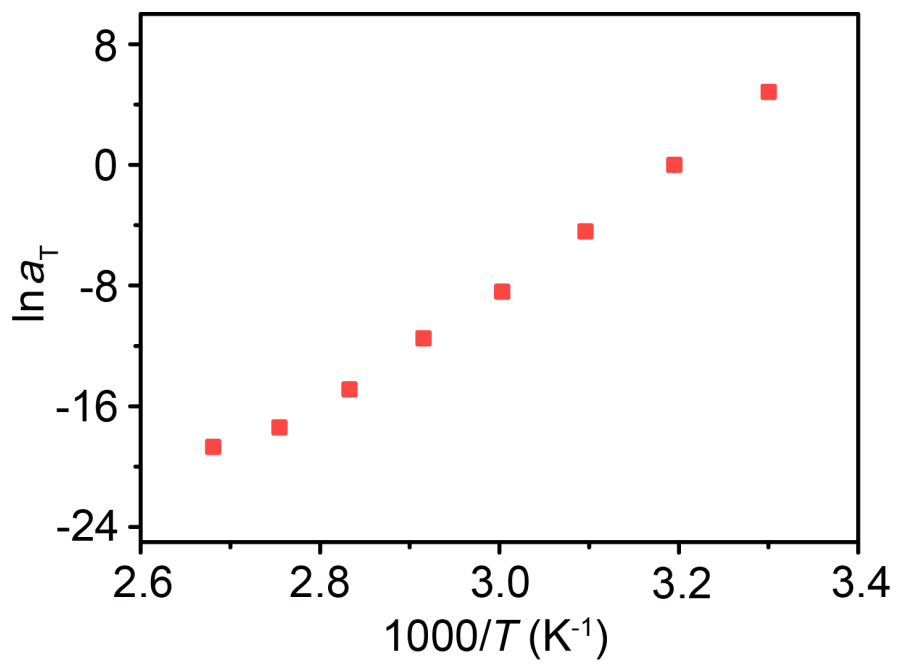

Figure S44. Temperature dependence of horizontal shift factors $\alpha_{\mathrm{T}}$ of the master curves of CSP2 at a reference temperature of $40^{\circ} \mathrm{C}$.

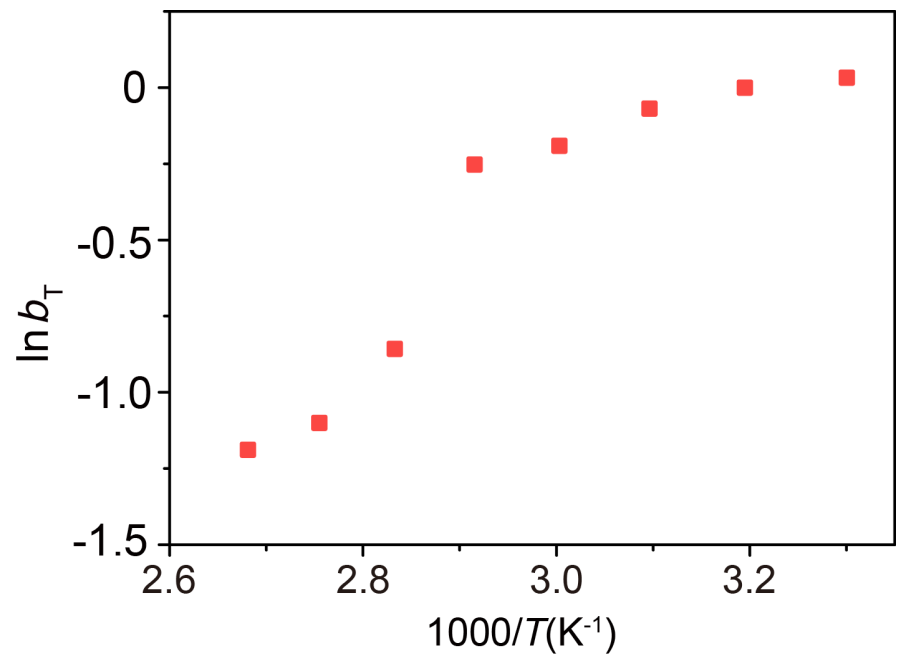

Figure S45. Temperature dependence of vertical shift factors $b_{\mathrm{T}}$ of the master curves of CSP-2 at a reference temperature of $40{ }^{\circ} \mathrm{C}$. 

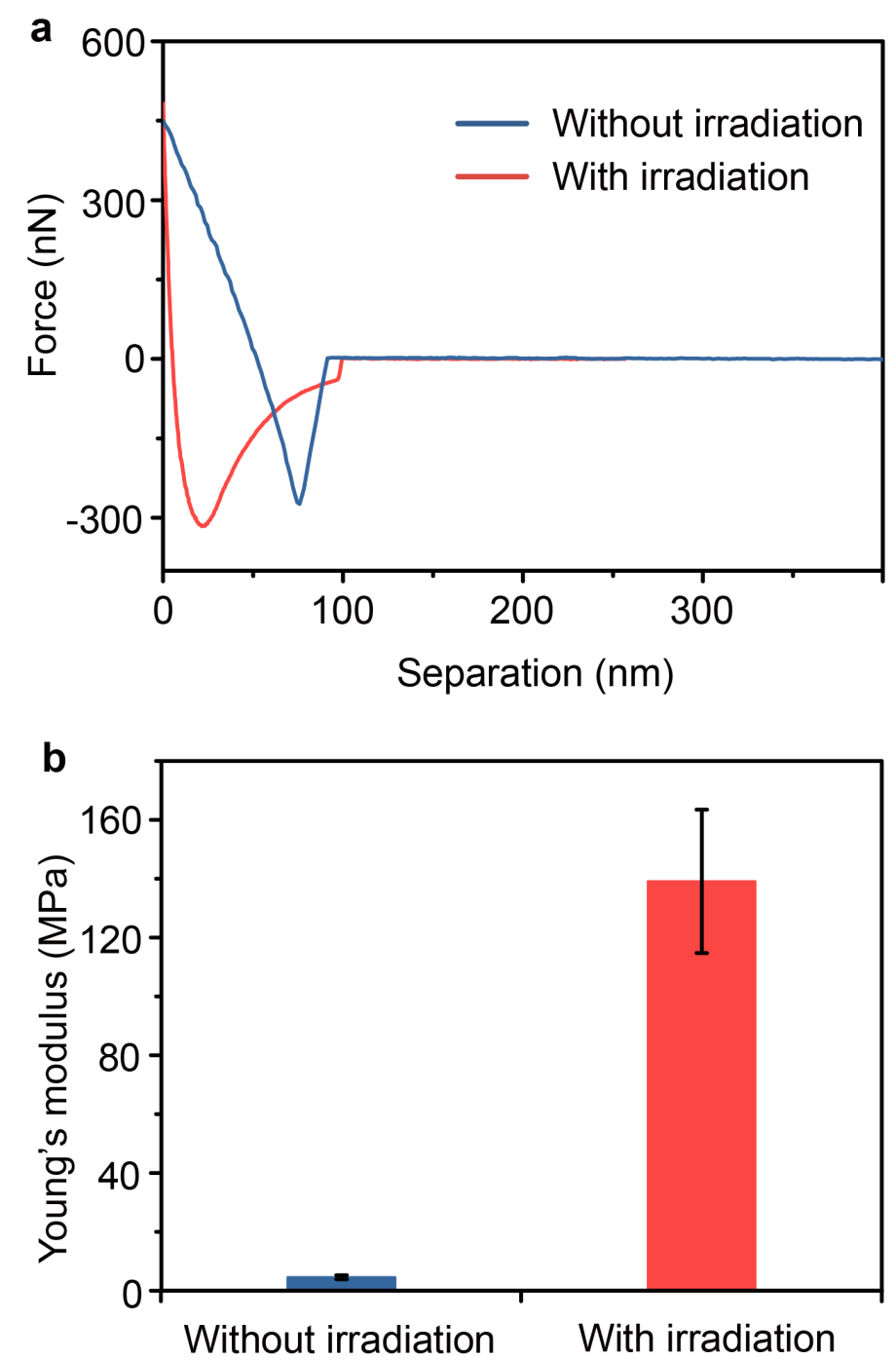

Figure S46. (a) Force separation curves of the films without and with irradiation during the annealing process at $50{ }^{\circ} \mathrm{C}$. (b) Young's moduli extracted from the force separation curves of the film without and with irradiation. As shown above, when photo-irradiation and heating of $50{ }^{\circ} \mathrm{C}$ were simultaneously applied to a film, the Young's moduli of the film after $2 \mathrm{~h}$ irradiation was up to $139.1 \mathrm{MPa}$ which was higher than that obtained by irradiation at room temperature (104.3 MPa). This could be interpreted that the produced uncovering UPy cannot completely combine with DAN group because their motion in solid state is restricted in a certain degree whereas the high temperature could promote their motion. 


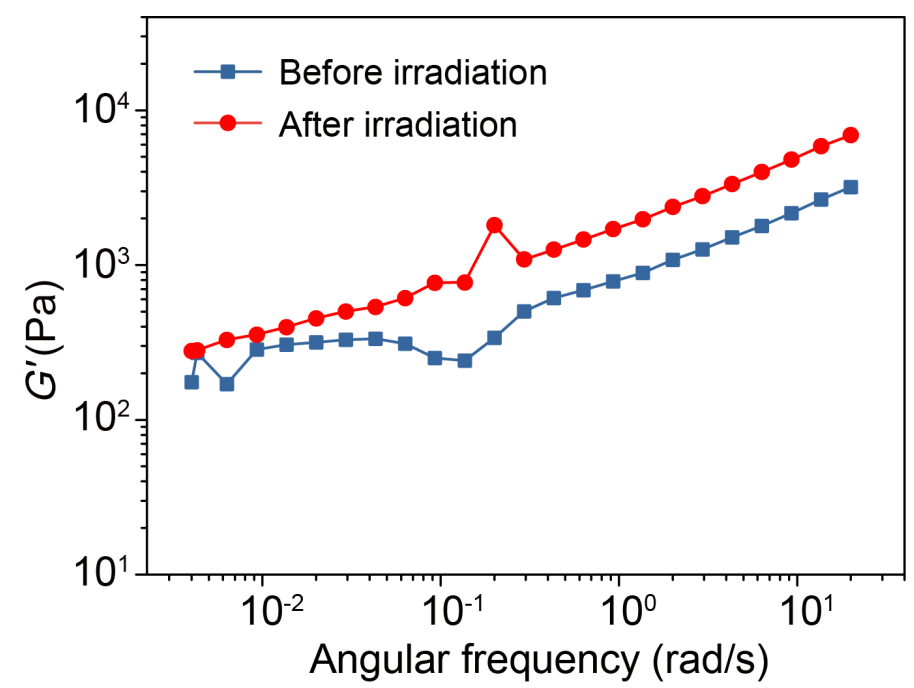

Figure S47. Storage modulus $\left(G^{\prime}\right)$ versus frequency $(\omega)$ for the CSP-2 film before and after irradiation. These results were record before and after the in situ rheological measurements of photo-induced combination of CPs and SPs in the solid state.

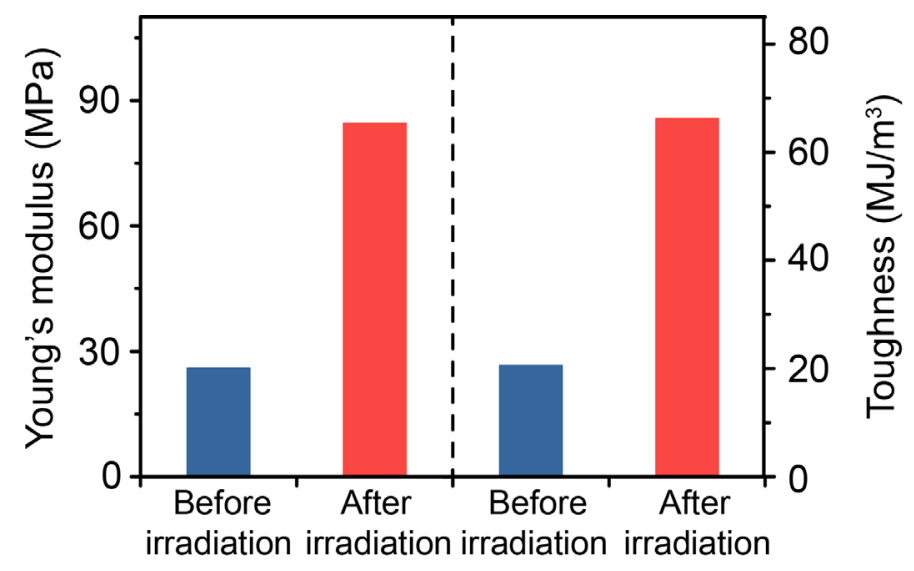

Figure S48. Young's moduli and toughness calculated based on the stress-strain curves of the film before and after irradiation. 


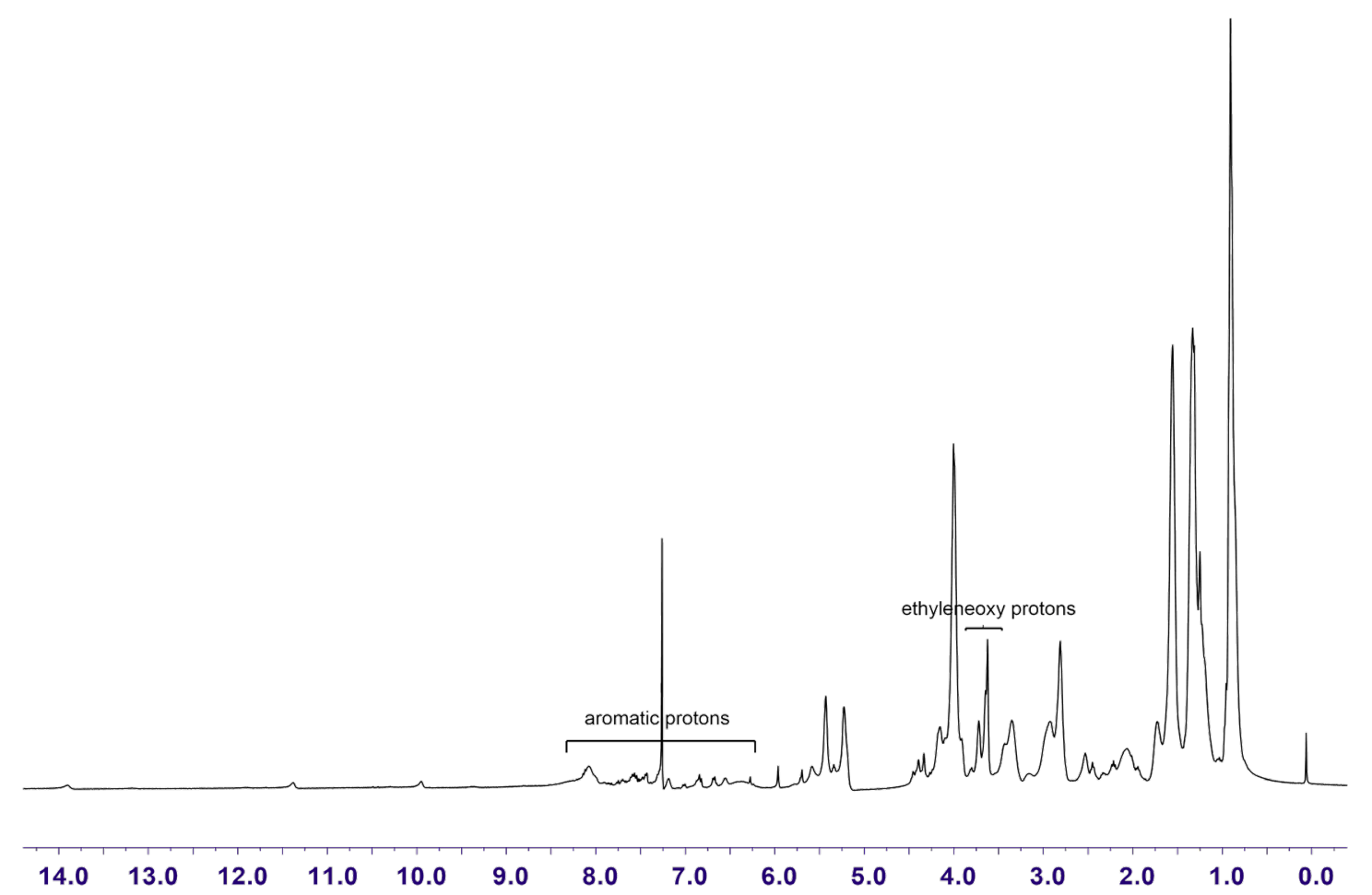

Figure S49. ${ }^{1} \mathrm{H}$ NMR spectrum $\left(\mathrm{CDCl}_{3}\right.$, room temperature, $\left.400 \mathrm{MHz}\right)$ of the mixture of $\mathrm{CPs}$ and SPs in the solid state after a $2 \mathrm{~h}$ irradiation. 
a
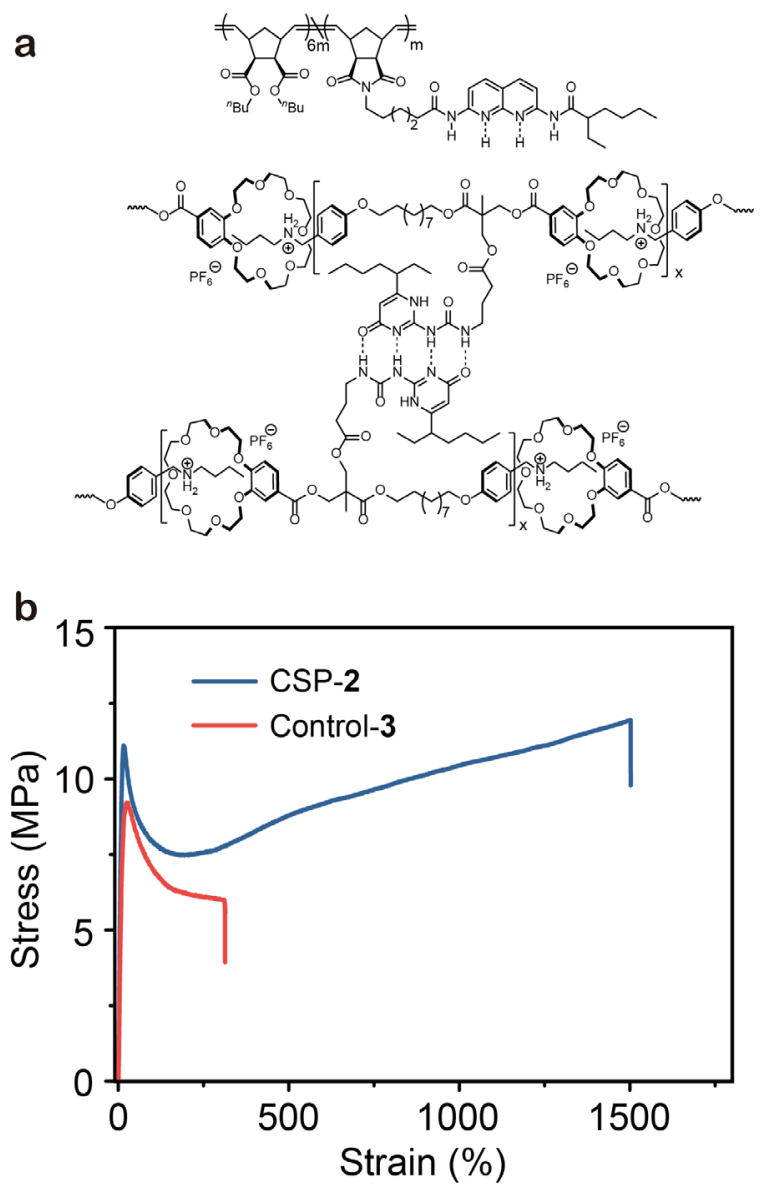

Figure S50. (a) Chemical structure of control-3 in which DAN moiety on the CPs is acidized, and doesn't involve in the formation of hetero-quadruple H-bonding. Without the competition of DAN, the deprotected UPy moiety only forms self-complementary dimer. (b) Stress-strain curves of CSP-2 and control-3 recorded with a deformation rate of $100 \mathrm{~mm} / \mathrm{min}$. The purpose to design this control was to confirm that the irradiation-enhanced mechanical properties of the mixed CPs and SPs in the solid state mainly resulted from the formation of UPy-DAN heterodimer rather than the UPy self-complementary dimer. As shown in Figure S50b, the crosslinked SPs could enhance the stiffness of the material but the ductility of the specimen was poor with the strain at break of about $310 \%$. This value was not only much lower than that of CSP2, but also much lower than that shown by the mixed CPs and SPs after irradiation in the solid state (Figure 6e). Hence, the irradiation-enhanced mechanical properties of the CPs and SPs mixture are not due to the self-crosslinking of the SPs, but more likely arise from the combination of the CPs and SPs. 


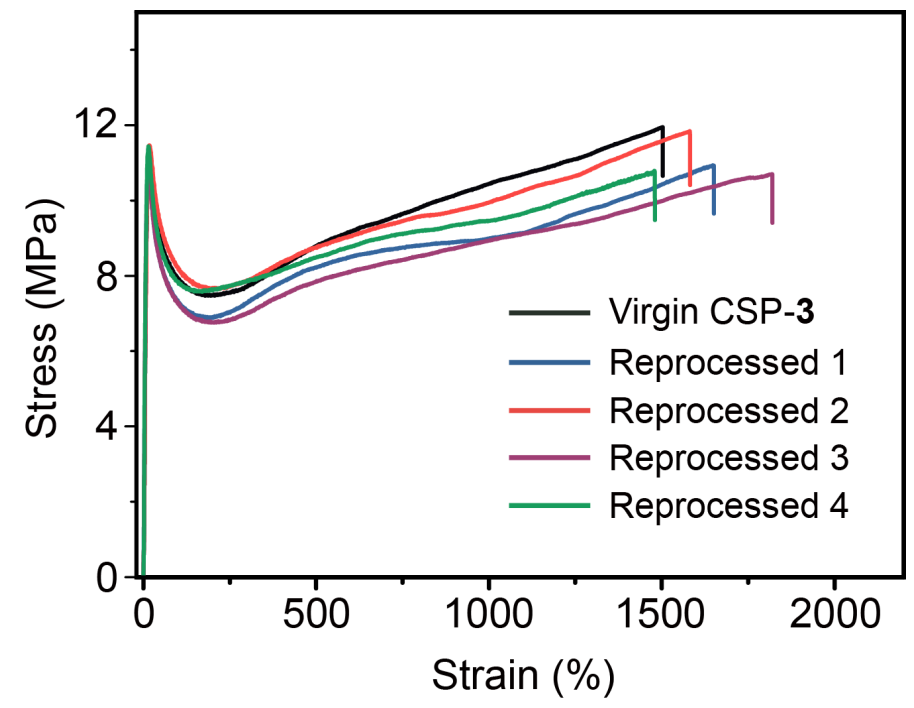

Figure S51. Stress-strain curves of CSP-2 after multiple cycles of recycle. The stress-strain curves of the reprocessed samples only changed slightly after four cycles, which indicates that CSP-2 has good malleability. 


\section{References:}

S1. Anderson, C. A.; Taylor, P. G.; Zeller, M. A.; Zimmerman, S. C. Room Temperature, CopperCatalyzed Amination of Bromonaphthyridines with Aqueous Ammonia. J. Org. Chem. 2010, 75, 4848-4851.

S2. Yu, Y. G.; Chae, C. G.; Kim, M. J.; Seo, H. B.; Grubbs, R. H.; Lee, J. S. Precise Synthesis of Bottlebrush Block Copolymers from $\omega$-End-Norbornyl Polystyrene and Poly(4-tert-butoxystyrene) via Living Anionic Polymerization and Ring-Opening Metathesis Polymerization. Macromolecules 2018, 51, $447-455$.

S3. Zheng, B.; Zhang, M.; Dong, S.; Liu, J.; Huang, F. A Benzo-21-Crown-7/Secondary Ammonium Salt [c2]Daisy Chain. Org. Lett. 2012, 14, 306-309.

S4. Yu, L.; Cao, R.; Yi, W.; Yan, Q.; Chen, Z.; Ma, L.; Song, H. Design, Synthesis and Evaluation of Difunctionalized 4-Hydroxybenzaldehyde Derivatives as Novel Cholinesterase Inhibitors. Chem. Pharm. Bull. 2010, 58, 1216-1220.

S5. Keizer, H. M.; González, J. J.; Segura, M.; Prados, P.; Sijbesma, R. P.; Meijer, E. W.; de Mendoza, J. Self-Assembled Pentamers and Hexamers Linked through Quadruple-Hydrogen-Bonded 2-Ureido4[1H]-Pyrimidinones. Chem. Eur. J. 2005, 11, 4602-4608.

S6. Fischer, T. S.; Schulze-Sünninghausen, D.; Luy, B.; Altintas, O.; Barner-Kowollik, C. Stepwise Unfolding of Single-Chain Nanoparticles by Chemically Triggered Gates. Angew. Chem. Int. Ed. 2016, $55,11276-11280$.

S7. Song, P.; Xu, Z.; Lu, Y.; Guo, Q. Bio-Inspired Hydrogen-Bond Cross-Link Strategy toward Strong and Tough Polymeric Materials. Macromolecules 2015, 48, 3957-3964.

S8. Hentschel, J.; Kushner, A. M.; Ziller, J.; Guan, Z. Self-Healing Supramolecular Block Copolymers. Angew. Chem. Int. Ed. 2012, 51, 10561-10565.

S9. Yang, L.; Lin, Y.; Wang, L.; Zhang, A. The Synthesis and Characterization of Supramolecular Elastomers Based on Linear Carboxyl-Terminated Polydimethylsiloxane Oligomers. Polym. Chem. 2014, $5,153-160$.

S10. Chen, Y.; Guan, Z. Multivalent Hydrogen Bonding Block Copolymers Self-Assemble into Strong and Tough Self-Healing Materials. Chem. Commun. 2014, 50, 10868-10870.

S11. Hart, L. R.; Hunter, J. H.; Nguyen, N. A.; Harries, J. L.; Greenland, B. W.; Mackay, M. E.; S54 
Colquhoun, H. M.; Hayes, W. Multivalency in Healable Supramolecular Polymers: The Effect of Supramolecular Cross-Link Density on the Mechanical Properties and Healing of Non-Covalent Polymer Networks. Polym. Chem. 2014, 5, 3680-3688.

S12. Agnaou, R.; Capelot, M.; Tence-Girault, S.; Tournilhac, F.; Leibler, L. Supramolecular Thermoplastic with 0.5 Pa·s Melt Viscosity. J. Am. Chem. Soc. 2014, 136, 11268-11271.

S13. Mozhdehi, D.; Ayala, S.; Cromwell, O. R.; Guan, Z. Self-Healing Multiphase Polymers via Dynamic Metal-Ligand Interactions. J. Am. Chem. Soc. 2014, 136, 16128-16131.

S14. Cordier, P.; Tournilhac, F.; Soulié-Ziakovic, C.; Leibler, L. Self-Healing and Thermoreversible Rubber from Supramolecular Assembly. Nature 2008, 451, 977-980.

S15. Burnworth, M.; Tang, L.; Kumpfer, J. R.; Duncan, A. J.; Beyer, F. L.; Fiore, G. L.; Rowan, S. J.; Weder, C. Optically Healable Supramolecular Polymers. Nature 2011, 472, 334-338.

S16. Chen, Y.; Kushner, A. M.; Williams, G. A.; Guan, Z. Multiphase Design of Autonomic Self-Healing Thermoplastic Elastomers. Nat. Chem. 2012, 4, 467-472.

S17. Reisch, A.; Roger, E.; Phoeung, T.; Antheaume, C.; Orthlieb, C.; Boulmedais, F.; Lavalle, P.; Schlenoff, J. B.; Frisch, B.; Schaaf, P. On the Benefits of Rubbing Salt in the Cut: Self-Healing of Saloplastic PAA/PAH Compact Polyelectrolyte Complexes. Adv. Mater. 2014, 26, 2547-2551.

S18. Wang, D.; Guo, J.; Zhang, H.; Cheng, B.; Shen, H.; Zhao, N.; Xu. J. Intelligent Rubber with Tailored Properties for Self-Healing and Shape Memory. J. Mater. Chem. A 2015, 3, 12864-12872.

S19. Lewin, M.; Pearce, E. M. Handbook of fiber chemistry; Marcel Dekker: New York, 1998.

S20. Gosline, J. M.; Guerette, P. A.; Ortlepp, C. S.; Savage, K. N. The Mechanical Design of Spider Silks: from Fibroin Sequence to Mechanical Function. J. Exp. Biol. 1999, 202, 3295-3303.

S21. Elices, M.; Plaza, G. R.; Pérez-Rigueiro, J.; Guinea, G. V. The Hidden Link between Supercontraction and Mechanical Behavior of Spider Silks. J. Mech. Behav. Biomed. Mater. 2011, 4, $658-669$.

S22. Lin, P.; Ma, S.; Wang, X.; Zhou, F. Molecularly Engineered Dual-Crosslinked Hydrogel with Ultrahigh Mechanical Strength, Toughness, and Good Self-Recovery. Adv. Mater. 2015, 27, 2054-2059. S23. Yang, Y.; Wang, X.; Yang, F.; Shen, H.; Wu, D. A Universal Soaking Strategy to Convert Composite Hydrogels into Extremely Tough and Rapidly Recoverable Double-Network Hydrogels. Adv. Mater. 2016, 28, 7178-7184.

S24. Gao, G.; Du, G.; Sun, Y.; Fu, J. Self-Healable, Tough, and Ultrastretchable Nanocomposite S55 
Hydrogels Based on Reversible Polyacrylamide/Montmorillonite Adsorption. ACS Appl. Mater. Interfaces 2015, 7, 5029-5037.

S25. Kushner, A. M.; Vossler, J. D.; Williams, G. A.; Guan, Z. A biomimetic Modular Polymer with Tough and Adaptive Properties. J. Am. Chem. Soc. 2009, 131, 8766-8768.

S26. Rao, Y.; Chortos, A.; Pfattner, R.; Lissel, F.; Chiu, Y.; Feig, V. R.; Xu, J.; Kurosawa, T.; Gu, X.; Wang, C.; He, M.; Chung, J. W.; Bao, Z. Stretchable Self-Healing Polymeric Dielectrics Cross-Linked Through Metal-Ligand Coordination. J. Am. Chem. Soc. 2016, 138, 6020-6027.

S27. Filippidi, E.; Cristiani, T. R.; Eisenbach, C. D.; Waite, J. H.; Israelachvili, J. N.; Ahn, B. K.; Valentine, M. T. Toughening Elastomers Using Mussel-Inspired Iron-Catechol Complexes. Science 2017, $358,502-505$.

S28. Wu, J.; Cai, L. H.; Weitz, D. A. Tough Self-Healing Elastomers by Molecular Enforced Integration of Covalent and Reversible Networks. Adv. Mater. 2017, 29, 1702616.

S29. Yan, X.; Liu, Z.; Zhang, Q.; Lopez, J.; Wang, H.; Wu, H. C.; Niu, S.; Yan, H.; Wang, S.; Lei, T.; Li, J.; Qi, D.; Huang, P.; Huang, J.; Zhang, Y.; Wang, Y.; Li, G.; Tok, J. B.-H.; Chen, X.; Bao, Z. Quadruple H-Bonding Cross-Linked Supramolecular Polymeric Materials as Substrates for Stretchable, Antitearing, and Self-Healable Thin Film Electrodes. J. Am. Chem. Soc. 2018, 140, 5280-5289.

S30. Song, Y.; Liu, Y.; Qi, T.; Li, G. L. Towards Dynamic but Supertough Healable Polymers through Biomimetic Hierarchical Hydrogen-Bonding Interactions. Angew. Chem. Int. Ed. 2018, 57, $13838-13842$.

S31. Zhang, L.; Liu, Z.; Wu, X.; Guan, Q.; Chen, S.; Sun, L.; Guo, Y.; Wang, S.; Song, J.; Jeffries, E. M.; He, C.; Qing, F.-L.; Bao, X.; You, Z. A Highly Efficient Self-Healing Elastomer with Unprecedented Mechanical Properties. Adv. Mater. 2019, 31, 1901402.

S32. Zhang, C.; Yang, Z.; Duong, N. T.; Li, X.; Nishiyama, Y.; Wu, Q.; Zhang, R.; Sun, P. Using Dynamic Bonds to Enhance the Mechanical Performance: From Microscopic Molecular Interactions to Macroscopic Properties. Macromolecules 2019, 52, 5014-5025. 